\title{
A Model for Shear Response in Swimming Plankton
}

\author{
by \\ Justin Shaw
}

\author{
A thesis \\ presented to the University of Waterloo \\ in fulfillment of the \\ thesis requirement for the degree of \\ Master of Mathematics \\ in \\ Applied Mathematics
}

Waterloo, Ontario, Canada, 2016

(C) Justin Shaw 2016 
I hereby declare that I am the sole author of this thesis. This is a true copy of the thesis, including any required final revisions, as accepted by my examiners.

I understand that my thesis may be made electronically available to the public. 


\begin{abstract}
The responses of zooplankton to the hydrodynamic variables of their environment, such as shear, are not well understood. We examine stochastic swimming models of the run and tumble type for plankton moving in a velocity field induced by internal waves in a channel. The swimming of individual plankton is modelled as a random walk, modified to include a shear response and a biased swimming towards a preferred light level. An inertial particle model is developed from first principles, and compared to a particle model based on the integral curve definition. In all, twelve particle models for the motion of plankton under advection and their own propulsion are considered. The model which includes the forces of gravity, biased swimming, and a freeze in shear response predicts aggregation of plankton populations along the bottom boundary of high shear regions. It is also shown that all models considered predict vertical patches.
\end{abstract}




\section{Acknowledgements}

Thanks to Marek Stastna for his supervision and patience. This work was funded by a grant from the National Sciences and Engineering Research Council of Canada (RGPIN311844-37157). Special thanks to Jared Penney for assistance in editing. 


\section{Dedication}

To Marek, for making me think, and to Science, for keeping me company. 


\section{Table of Contents}

Author's Declaration $\quad$ ii

$\begin{array}{ll}\text { Abstract } & \text { iii }\end{array}$

Acknowledgements $\quad$ iv

Dedication $\quad$ v

List of Figures $\quad$ ix

List of Tables $\quad$ xiii

1 Introduction $\quad 1$

1.1 Some Background . . . . . . . . . . . . . . . . . . 1

1.2 The Euler Equations and Linear Internal Waves . . . . . . . . . . . . . 5

1.3 Model Initialization . . . . . . . . . . . . . . . . . . . . . . . . . 9

2 Modeling the Motion of Plankton $\quad 10$

2.1 Particle Models: . . . . . . . . . . . . . . . . . . . . . . 10

2.1.1 An Overview of Particle Models: . . . . . . . . . . . . . . . 10

2.1.2 The Fluid Particle Model . . . . . . . . . . . . . . . . . . . . . . . 13

2.1.3 A Particle Model with Inertia . . . . . . . . . . . . . . . . . . 14 
2.1.4 The Stokes' Inertia Model . . . . . . . . . . . . . . . . . . . . 16

2.1.5 The Effects of Relative Density . . . . . . . . . . . . . . . 19

2.2 Swimming: . . . . . . . . . . . . . . . . . . . . . . . 23

2.2.1 The (Discrete) Run and Tumble Model . . . . . . . . . . . . . . . 23

2.2.2 The Fluid Particle Model with Run and Tumble . . . . . . . . . . . 25

2.2 .3 Stokes' Inertia with Run and Tumble . . . . . . . . . . . . . . 25

2.2.4 The Effects of Density under Run and Tumble . . . . . . . . . . 26

2.2.5 Stokes' Inertia with Run and Tumble: Our Specific Case . . . . . 27

2.2 .6 Biased Swimming . . . . . . . . . . . . . . . . . . . . . . . 29

2.3 Completing the Models . . . . . . . . . . . . . . . . . . . . . . . 30

2.3 .1 Plankton Response to Shear . . . . . . . . . . . . . . . . 30

2.3.2 Theoretical Concerns . . . . . . . . . . . . . . . . . . . . . 33

3 Results $\quad 35$

3.1 Setup . . . . . . . . . . . . . . . . . . . . 35

3.1 .1 Parameter Space . . . . . . . . . . . . . . . . . . 35

3.1 .2 Model Selection . . . . . . . . . . . . . . . . . . . . . . . . . . . 39

3.1 .3 Tools . . . . . . . . . . . . . . . . . . . . . . . . . 40

3.2 Analysis of the Mechanisms . . . . . . . . . . . . . . . . . . 41

3.2.1 Comparing the Fluid Particle and Neutrally Buoyant Stokes' Inertia Models . . . . . . . . . . . . . . . . . . . . . . . . 44 41

3.2 .2 Trapping: Base Models . . . . . . . . . . . . . . . . . . . . 43

3.2 .3 Trapping: Biased Swimming . . . . . . . . . . . . . . . . 44

3.2.4 Trapping: Shear Response . . . . . . . . . . . . . . . . . . 45

3.2.5 Trapping: Biased Swimming and Shear Response _ . . . . . . . 47

3.2 .6 Trapping in Gyres . . . . . . . . . . . . . . . 47

3.2 .7 Light Attractors . . . . . . . . . . . . . . . . . 47

3.2 .8 Shear Attractors . . . . . . . . . . . . . . . . . . 50 
3.2 .9 Shear and Light Attractors . . . . . . . . . . . . . . . . . 52

3.3 Aggregation and Vertical Patch Formation . . . . . . . . . . . . 56

3.3.1 Aggregation Below Regions of High Shear . . . . . . . . . . . 56

$3.3 .2 \quad$ Vertical Patch Formation . . . . . . . . . . . . . . . . . 57

4 Discussion $\quad 59$

4.1 Directions for Future Research . . . . . . . . . . . . . . . . . . . . 59

4.1 .1 Light sensing in Zooplankton . . . . . . . . . . . . . . . 59

4.1 .2 Modelling . . . . . . . . . . . . . . . . . . . . 60

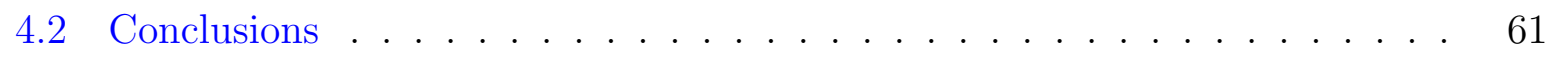

$\begin{array}{ll}\text { APPENDICES } & 67\end{array}$

4.3 Model Values . . . . . . . . . . . . . . . . . . . . . . . . 67

4.4 Classical Analysis: Adding Fluid Drift to Run and Tumble . . . . . . . . 68

4.4.1 The Diffusion Constant: One Dimensional Derivation . . . . . . . 68

4.4.2 The Diffusion Constant: Three Dimensional Derivation . . . . . . . 69

4.4.3 Modified Run and Tumble Models: Run and Tumble with Fluid Drift 71

4.4.4 FPE for Run and Tumble with Fluid Drift . . . . . . . . . 72

4.4.5 Re-evaluating the Model . . . . . . . . . . . . . . . . . . 73

4.4.6 Run and Tumble with Fluid Drift and Shear Response . . . . . . 74

4.4 .7 Conclusion . . . . . . . . . . . . . . . . . 77

4.5 On the Inadequacy of Variance for our Problem $\ldots \ldots \ldots \ldots$

$\begin{array}{ll}\text { References } & 80\end{array}$ 


\section{List of Figures}

1.1 A schematic of the tidally driven, coherent motions near a large amplitude sill such as the one at Knight Inlet. Large amplitude internal waves form in the lee of the sill. Smaller scale shear instabilities are not shown. The accelerated flow downslope is indicated by a green arrow. This flow induces separation from the bottom boundary (indicated by the dotted line and the blue arrow beneath it), which in turn leads to shear instability and turbulence production. . . . . . . . . . . . . . . . .

1.2 The stream function solution of the linear internal wave equation: the flow is along lines of constant color where the red gyres are counterclockwise and the blue gyres are clockwise. The whole map moves slowly from left to right. The initial position of the plankton is shown as a white line. . . . . . . .

2.1 A hierarchy of possible particle models. Here the dotted arrows mean "simplify" and the solid arrows mean "complicate." . . . . . . . . . . . . .

2.2 The paths followed by a fluid particle (in blue) and a particle with inertia (in red), released from the origin. The inertial particle is initially at rest, and is gradually accelerated to match the speed of the flow $\mathbf{u}=(0.1,0)$. The fluid particle matches the flow exactly. . . . . . . . . . . . . .

2.3 The trajectory of an inertialess particle in blue matches a randomly changing u. In red, a particle with inertia initially at rest at the origin is gradually accelerated to match the speed of the flow, but with every change of the flow's direction we get a new acceleration. For this simulation we took the flow's magnitude to be constant, with randomly changing direction. It is clear that the inertia acts to smooth the particle path. . . . . . . . . .

2.4 a) The discrete run and tumble model of plankton swimming. b) A variant of run and tumble where the plankton do not swim if the shear is too high. 
2.5 A spacetime plot of the fluid particle model with the freeze in shear response. The smooth curves from the linedrop at $t=0$ correspond to plankton which are frozen in shear, and so are deterministically advected by the flow, whereas the jagged curves correspond to the plankton in low shear which swim as they are advected. This effect can be seen to vary over time with the trajectory of any given plankter, as they switch back and forth between swimming and freezing in shear. . . . . . . . . . . . . .

3.1 Fraction of the domain considered high shear as a function of $s$ in centimeters. Lighter curves correspond to smaller critical shear values. All curves asymptote to filling the domain with high shear, but curves for larger critical values do so more slowly. . . . . . . . . . . . . . . . . . . . .

3.2 Red represents high shear regions. From left to right these are 25\%, 50\%, and $75 \%$ high shear quantiles. . . . . . . . . . . . . . . .

3.3 The initial position of the plankton is shown in the first panel, the second panel is at $t=8 \mathrm{~s}$, the third is at $t=56 \mathrm{~s}$. This is the base fluid particle model with swimming, outlined in section 2.2.2. This flow is the first quantile of the $5 \times 10^{-4}$ critical value, so a medium speed flow from the regime. The red gyres are counterclockwise and the blue gyres are clockwise which clearly advects the plankton cloud as they swim. . . . . . . . . . . . . . .

3.4 Red is high shear and blue is low shear. Panel a) shows inertia carrying a white plankter modelled under the neutrally buoyant Stokes' inertia model out of a high shear region while a plankter modelled under the fluid particle (in green) follows the integral curve and stays inside. Panel b) shows inertia keeping a plankter inside a high shear region. . . . . . . . . . . . . . . .

3.5 The fluid particle model is shown in magenta, the neutral buoyancy Stokes' inertia in yellow, and the Stokes inertia in green. In the left panel $(t=3000$ s) we see all three populations caught below a region of high shear, shown in black. As they swim up they are advected by the high shear zone and the net effect is that they are essentially trapped despite being predomininantly outside the high shear region. The right panel $(t=3200 \mathrm{~s})$ shows the Stokes inertia model trapped between the bottom boundary and a high shear region enclosing it. . . . . . . . . . . . . . . . . . . . . 
3.6 We see patches of all three populations, in black, white, and magenta, in the form of two unstable and stable light attractor pairs. Times from left to right are 2396, 2596, and $3356 \mathrm{~s}$. The unstable light attractors in the middle two gyres feed the stable light attractors in the outer two gyres, and in the end the left unstable attractor has almost been destroyed by this process. Note the horizontal axes: this process occurs in the Lagrangian frame. . . .

3.7 The top left panel $(t=1000 \mathrm{~s})$ shows patch formation by ring shaped shear attractors for all populations: fluid particle in magenta, neutrally buoyant Stokes' inertia in white, and Stokes' inertia in black. Note the trapping in a band as well around $x=-25$. The top right and bottom panels $(t=1200 \mathrm{~s})$ show filament shear attractors. The third panel shows the the shear field plotted at the same time as the second panel, high shear in black and low shear in white. The color of neutrally buoyant Stokes' inertia has been changed from white to yellow and the color of Stokes' inertia has been changed from black to green. This panel clearly shows the separation of the Stokes' inertia model (green) from the other two (yellow and magenta), as well as trapping below the high shear region. . . . . . . . . . . . . . . .

3.8 The four basic possibilities of a plankter with a preferred light level leaving a high shear region. Red is high shear and blue is low shear. . . . . . . . .

3.9 The left panel $(t=796 \mathrm{~s})$ shows an unstable and stable attractor pair on the left, and on the right a shear and light attractor containing $z_{L}$. The right panel $(t=1356 \mathrm{~s})$, is after the unstable attractor has been destroyed. The light attractor containing $z_{L}$ has shrunk to match the evolution of the high shear region. . . . . . . . . . . . . . . .

3.10 Both panels $(t=3920 \mathrm{~s})$ show the aggregation of plankton along the bottom boundary of high shear regions as predicted by the Stokes' inertia model (black in left, green in right). The left panel shows the stream function and the right shows high shear in black. Note the horizontal separation of the fluid particle and neutral buoyancy Stokes' inertia model from the Stokes' inertia model. This substantial separation is a result of gravity acting to separate the populations in the vertical so that the populations are at different locations as the shear regions evolve. In this case the Stokes' inertia model has led to about half the plankton aggregating below high shear, and the other half in two groups at the preferred light level on either side of the other two models. . . . . . . . . . . . . . . . . . 
3.11 Two examples of vertical patch formation in the base models. The left panel $(t=200 \mathrm{~s})$ shows the fastest flow tested. Patches are formed by trapping in gyres, as all populations curl around the blue gyres, and are fairly dense in the central red gyre. Also note the relatively sharp vertical bands at $x=-125,100 \mathrm{~m}$. The right panel $(t=1400 \mathrm{~s})$ shows the slowest flow where trapping occurred. The background is $u(x, z, t)$, and we clearly see the Stokes' inertia model in black separated to the bottom of the channel and trapped in the horizontal. . . . . . . . . . . . . . . . . 


\section{List of Tables}

3.1 Values of $s$ corresponding to high shear area quantiles for the four critical values tested are shown in panel a). Mean vertical fluid speeds over the domain at $t=0$ are shown in panel $\mathrm{b}$ ), by the definition in the text, these values show that for this parameter space exploration the settling regime consists of the slowest five flows. . . . . . . . . . . . . . . . . .

3.2 Maximum speeds at $t=0 \mathrm{in} \mathrm{m} / \mathrm{s}$ are shown in panel a). Max values of $\left|I_{2}\right|$ over the domain at $t=0$ are shown in panel b). Note the max shear is smaller than our accuracy in the first row of b). . . . . . . . . . . .

3.3 We consider models corresponding to all possible ways of filling out this chart, so each model has the four choices of no freeze or biased swimming, both, or only one of freeze or biased swimming, for a total of 12 models. . .

3.4 Maximum values of the components of the velocity field at $t=0$. Panel (a) shows the horizontal component and (b) shows the vertical. By smoothness and periodicity we take these values to be representative of the values over the entire time domain. . . . . . . . . . . . . . . . . . . .

3.5 Maximum values of the components of the velocity field at $t=0$ and $z=7$ m. Panel (a) shows the horizontal component and (b) shows the vertical. By smoothness we take these values to be representative of the values near $z=7$ as well. By periodicity we take these values to be representative of the values over the entire time domain. . . . . . . . . . . . . . .

4.1 The amplitudes $s$ for the streamfunction vary according to the scheme presented in 3.1 of 3.1.1. The model parameters which are held constant are presented here. The Peclet numbers and diffusion constant are for the base fluid particle model. The inertial model diffusion constant should be similar, with the inertial effects averaging out, but I have not included a proof of this fact. . . . . . . . . . . . . . . . . . . 


\section{Chapter 1}

\section{Introduction}

\subsection{Some Background}

Water-dwelling organisms continually encounter characteristics of the flow they may find undesirable. It is theorized that some species of copepods may avoid regions of high turbulence or shear [39]. The reasons for this response are also theoretical: turbulence could potentially enhance encounter rates of predators and prey, which could lead prey to avoid these regions [49], turbulence may make swimming difficult, or high shear could simply be uncomfortable [61]. This aversion to high shear regions would change the distribution of the population as the individuals in that population avoid those conditions which they find undesirable.

Several studies have considered the interaction of upward swimming plankton with the passage of internal waves. Stastna et al. [56] considered the interaction of internal solitary waves with plankton using Lagrangian models based on the Langevin equation. In the absence of a biased swimming behaviour, plankton followed a diffusion process and hence observations showing an increased concentration of plankton at certain depths were impossible to reproduce. For plankton which had a preferred light level, and that would swim vertically to maintain that light level, a fully nonlinear internal solitary wave of depression would advect plankton downward as they passed through the wavefront, causing the plankton to swim upwards to return to their preferred light level. As the plankton population passed the wave's crest, the wave-induced currents forced it upward again. Combined with the swimming of the plankton, this led the population to overshoot their preferred light level, creating an aggregation region at the rear of the wave and above 
the preferred light level. After the wave had passed, the plankton slowly drifted down to their preferred light level. Lennert-Cody and Franks [35] also discussed perturbation from a preferred light level from an Eulerian perspective with linear and weakly nonlinear waves in a two layer fluid. They found that the interaction of internal waves and swimming plankton could produce along-isopycnal patchiness in plankton blooms. Scotti and Pineda [52] found that swimming upwards as a response to downwelling currents associated with gravity currents allows for Lagrangian transport of plankton over large distances. All of these examples illustrate that the passing of internal waves can have a direct effect on the spatial distribution of plankton with preferred light levels.

The effect of shear on plankton populations has been studied before as well. Ianson et al. [25] noted that in Knight Inlet, British Columbia, Euphausia pacifica are observed below their preferred light level when close to a sill. The authors attempted to explain this with a variety of simulated shear responses, and chose a shear response of downward swimming as the model that most closely matched acoustic data. Gyrotactic phytoplankton were found to collect in a shear layer in [15], and aggregated as a result of interaction with a simple vortical flow in [14], leading to the prediction that motility characteristics may drive spatial cell distributions. An experimental example of this 'unmixing' was presented in [13], in which upward swimming produced nonuniform plankton distributions in a vortical flow. Gyrotactic trapping in a shear layer was examined in [24]. The primary effect of shear in these last four publications was as a trigger to reorient gyrotactic phytoplankton. Following [25], in this paper we examine the response of zooplankton to shear directly, sometimes called rheotaxis (see [46]).

Knight Inlet, British Columbia, Canada is a fjord with strong tidal flows driving internal wave generation over the large amplitude bottom topography of a glacial sill. It has been the site of a number of field studies, including the well-documented Knight Inlet Sill-Flows experiment [28], as well as a number of theoretical/numerical studies ([33], [2], [55], [11]). The flow over the Knight Inlet sill is extremely rich dynamically, yielding examples of flow separation from the bottom, shear instability, large amplitude breaking internal waves (see for example Figures 10 to 16 in [33]), as well as an upstream response that includes internal solitary waves [17], and significant spatiotemporal variation in the intensity of turbulence [29]. The field situation is further complicated by an asymmetry in the vertical stratification profile between the two sides of the sill [28], and significant transverse flows due to flow separation from the complex shoreline (Figures 6 and 7 of [27]). The coherent dynamical features near the sill are depicted schematically in Figure 1.1. Figures 10 to 16 of [33] provide a more dynamic picture based on simulations. It is important to note, that 
this is one point of view on the dynamics, one largely based on coherent structures such as large amplitude internal waves, with the temporal averages of the turbulent dissipation shown in Figure 3 of [29] offering a different point of view.

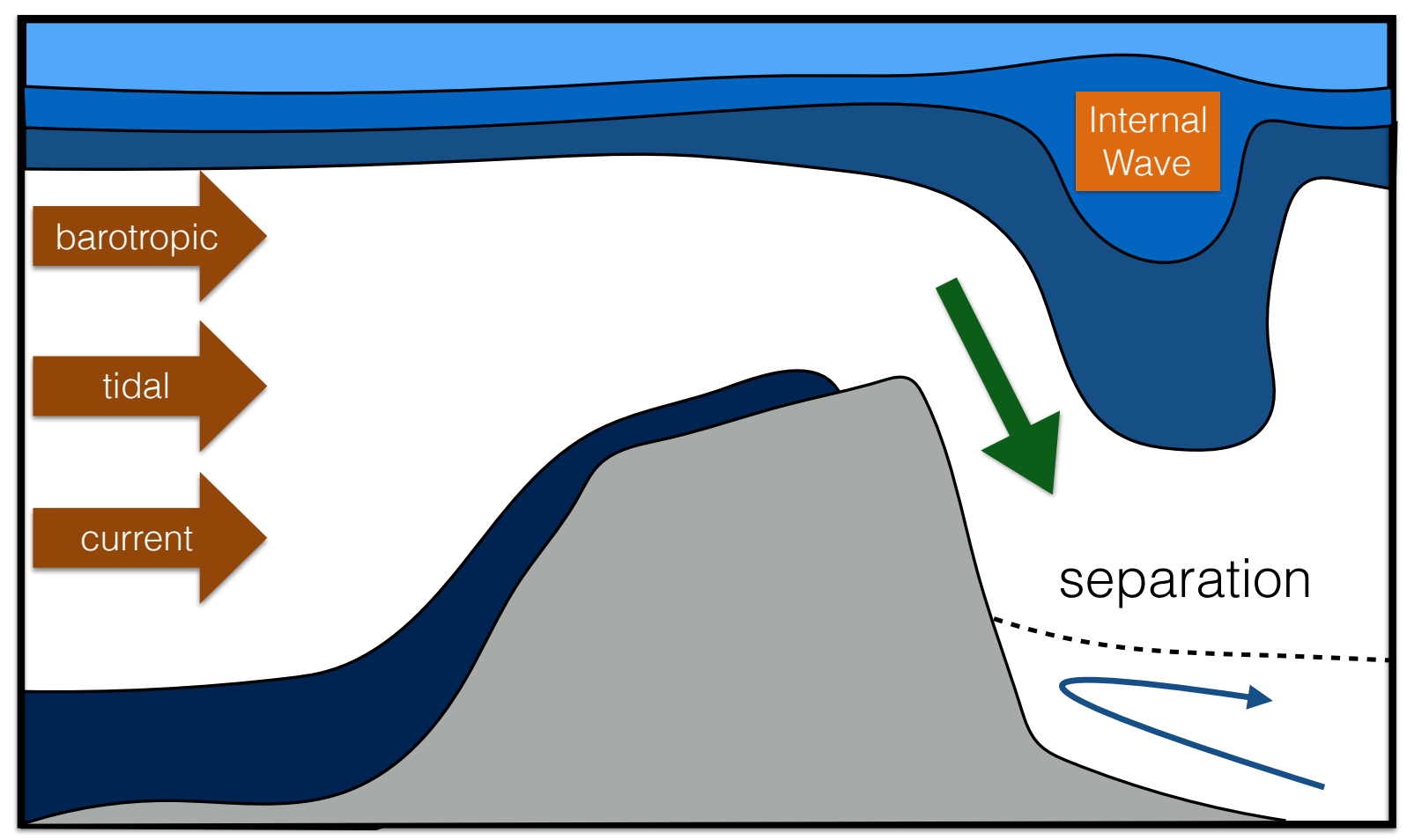

Figure 1.1: A schematic of the tidally driven, coherent motions near a large amplitude sill such as the one at Knight Inlet. Large amplitude internal waves form in the lee of the sill. Smaller scale shear instabilities are not shown. The accelerated flow downslope is indicated by a green arrow. This flow induces separation from the bottom boundary (indicated by the dotted line and the blue arrow beneath it), which in turn leads to shear instability and turbulence production.

A hierarchy of physical models can be employed to describe the effects of the ocean environment on plankton. Direct numerical simulations which resolve both turbulence and nonhydrostatic motions could be used to completely describe the stratified turbulent flow experienced by plankton on scales relevant to the plankton themselves. However the manner in which the turbulent flow is coupled to the complex shape of the plankton is beyond the present generation of numerical models. On larger scales, such as those associated with hydraulic flows in fjords [33] approximations are required in order for the model to handle 
the discrepancy between vertical horizontal scales as well as the full range of motions. As an example, the model employed by Lamb [33] uses a finite volume method that allows for an accurate representation of the non-hydrostatic flow over the Knight Inlet sill. This model has the drawback that it can only simulate a two-dimensional (along-fjord vs depth) slice. Larger scale models often make even more drastic approximations, most notably the hydrostatic approximation which neglects vertical acceleration. In the context of Knight Inlet the hydrostatic Hallberg Isopycnal Model was used by [28], and a modification of the two-dimensional, non-rotating, hydrostatic Princeton Ocean Model outlined in [10] was employed in [25]. While many of the commonly used approximations are successful in answering targeted scientific questions, it is important to not lose sight of the approximations, especially when attempting to link motions on different scales (e.g. internal waves and their effects on plankton). The model employed below is conceptual in the sense that it is simple to write down analytically, and well grounded in the physical oceanography literature since linear internal waves are commonly discussed in many introductory physical oceanography textbooks (e.g. [21], [53]). Linearity implies that we are free to superpose solutions, and beyond the understanding that linear theory is less and less accurate as amplitude increases, amplitude can be chosen at our discretion. This gives us a parameter to vary while we focus on the plankton's shear response mechanism. The flow should be contrasted with the internal solitary waves in Stastna et al. [56], which are exact solutions of the stratified Euler equations, but whose properties are difficult to tune a priori.

The idea that simple mechanistic responses of individuals heavily influence population distributions led us to construct the models outlined in this paper. These models consider both shear responses and preferred light levels from a Lagrangian (i.e. individual based) perspective. This work was inspired primarily by Ianson et al. [25]. In that work encountering the bottom boundary layer was synonymous with encountering shear, but shear can occur anywhere in fluid (e.g. due to shear instabilities). Thus, while the boundary layer will certainly contain some shear, near a sill boundary layer separation may transport a significant portion of this shear into the fluid interior as well by a process similar to that described in [41]. Shear can also occur with the passage of internal waves, which were not mentioned in [25], but have been documented at Knight Inlet by several authors [16]. We investigate the effect of a shear response occurring away from the boundary through a set of numerical models ${ }^{1}$. Throughout, we use Knight Inlet as the motivating example for flow parameters, and Euphausia pacifica as an example of zooplankton, but the principles presented are more general.

\footnotetext{
${ }^{1}$ All simulations were carried out in MATLAB.
} 
The remainder of the thesis is organized as follows. We discuss the Euler equations and linear internal waves, and describe the setup of our problem. We then develop several models for swimming particles from first principles, and compare and contrast those models, one of which is tuned to the parameters of the zooplankton species Euphausia pacifica. We review the results of our numerical experiments, which show that aggregations of Euphausia pacifica occur below regions of high shear, and that a wide variety of patches of plankton can develop in the vertical due to the passage of internal waves.

\subsection{The Euler Equations and Linear Internal Waves}

Flow over topography yields internal waves or billows which are observed away from the boundary (see [33], figures 10 to 16). Consider a flow in a two dimensional channel, representing a horizontal strip of the water column, which we will fill with linear internal waves representative of the actual flow. Label the $x$ axis as horizontal and the $z$ axis as vertical. Make the rigid lid assumption, giving us impermeable barriers at both $z=0$ and $z=H(=20 \mathrm{~m}$ in our case $)$. We employ the stratified Euler equations under the Boussinesq approximation, a standard approximation used in environmental and geophysical fluid dynamics because naturally occurring density changes are typically on the order of one percent or less [31]. These equations can be reduced to a single linear equation for channel flow as follows. We decompose the density as $\rho=\rho_{0}\left[\bar{\rho}(z)+\rho^{\prime}(x, y, z, t)\right]$, for a reference density $\rho_{0}$, and a non-dimensional stratification $\bar{\rho}(z)$ and perturbation $\rho^{\prime}$. Here $\rho^{\prime}$ is expected to be small compared to $\bar{\rho}$. We also write the pressure as $p=p_{H}(z)+p_{N H}(x, y, z, t)$ for the hydrostatic and nonhydrostatic pressure components. We then linearize around a state of rest, which means the reference frame is moving with the barotropic current. The linearized stratified Euler equations under the Boussinesq approximation for the two dimensional velocity field $(u, w)=(u(x, z, t), w(x, z, t))$ then reduce to

$$
\begin{aligned}
\rho_{0} \frac{\partial u}{\partial t} & =-\frac{\partial p_{N H}}{\partial x} \\
\rho_{0} \frac{\partial w}{\partial t} & =-\frac{\partial p_{N H}}{\partial z}-\rho_{0} \rho^{\prime} g \\
\frac{\partial u}{\partial x}+\frac{\partial w}{\partial z} & =0 \\
\frac{\partial \rho^{\prime}}{\partial t}+w \frac{\partial \bar{\rho}(z)}{\partial z} & =0 .
\end{aligned}
$$


They can be reduced to a single equation as follows: Cross differentiate equations (1.1) and (1.2) and introduce a stream function $\psi$ which we know is possible by equation (1.3). Using the density equation (1.4) and defining the buoyancy frequency as $N^{2}(z)=-g \frac{\partial \bar{\rho}}{\partial z}$ gives us

$$
\left(\nabla^{2} \psi\right)_{t t}+N^{2}(z) \psi_{x x}=0
$$

where subscripts denote partial derivatives. Assuming a traveling wave solution of the form $\psi=\exp [i(k x-\sigma t)] \phi(z)$ for wave number $k$, frequency $\sigma$, and vertical structure function $\phi$, substitution into (1.5) yields

$$
\phi_{z z}+\left(\frac{N^{2}(z)}{\sigma^{2}}-1\right) k^{2} \phi=0
$$

To make analytical progress we assume that the background stratification is linear so that the buoyancy frequency is constant, meaning $\bar{\rho}(z)=1-\Delta \rho z / H$ so that

$$
N^{2}(z)=-g \frac{\partial \bar{\rho}}{\partial z}=g \frac{\Delta \rho}{H} \equiv N_{0}^{2}
$$

By definition, this linear stratification means the density increases linearly with depth. This is in sharp contrast to the more physical choice of density change over the water column occurring in a relatively thin pycnocline. For an example of this type see [56], which considered the interaction of swimming plankton and internal solitary waves in a hyperbolic tangent stratification. Two layer models take this a step further, specifying a discontinuous jump in density across the pycnocline. For the case at hand, by letting $m^{2}=\left(\frac{N^{2}}{\sigma^{2}}-1\right) k^{2}$, substitution into (1.6) yields the simple harmonic oscillator

$$
\phi_{z z}+m^{2} \phi=0
$$

Since our boundaries at $z=0$ and $z=H$ are impermeable we specify no normal flow boundary conditions. With these conditions we obtain an analytic solution for the streamfunction in the form

$$
\psi(x, z, t)=\cos (k x-\sigma t) \sin (m z)
$$

where $m=n \pi / H$ and $\sigma=N k / \sqrt{k^{2}+m^{2}}$. The frequency is thus quantized. Notice that (1.5) is linear, so that we may also consider superpositions of solutions. The dispersion relation quantifies the fact that different modes travel at different speeds, and in particular higher vertical modes are slower. This means that each wave in a superposition of different 
vertical modes travels at a different speed. Linear internal waves have been studied in a variety of contexts but the most relevant to this paper is that of plankton patchiness [35]. For our experiments a superposition of two solutions is considered, a vertical mode one wave with a mode two perturbation, giving velocity field components of the form

$$
\begin{aligned}
u(x, z, t) & =A_{1} m_{1} \cos \left(k_{1} x-\sigma_{1} t\right) \cos \left(m_{1} z\right)+A_{2} m_{2} \cos \left(k_{2} x-\sigma_{2} t\right) \cos \left(m_{2} z\right) \\
w(x, z, t) & =A_{1} k_{1} \sin \left(k_{1} x-\sigma_{1} t\right) \sin \left(m_{1} z\right)+A_{2} k_{2} \sin \left(k_{2} x-\sigma_{2} t\right) \sin \left(m_{2} z\right)
\end{aligned}
$$

where $A_{i}, k_{i}, m_{i}, \sigma_{i}$ are all constants. The $k_{i}$ are the horizontal wavenumbers and the $m_{i}$ are the vertical wavenumbers. We also have $\sigma_{i}$ as the frequencies and the $A_{i}$ as the amplitudes. The values of $A_{i}$ are presented in 3.1.1, and the rest of the constants are presented in table 4.1 of 4.3. The flow geometry is presented in Figure 1.2. 


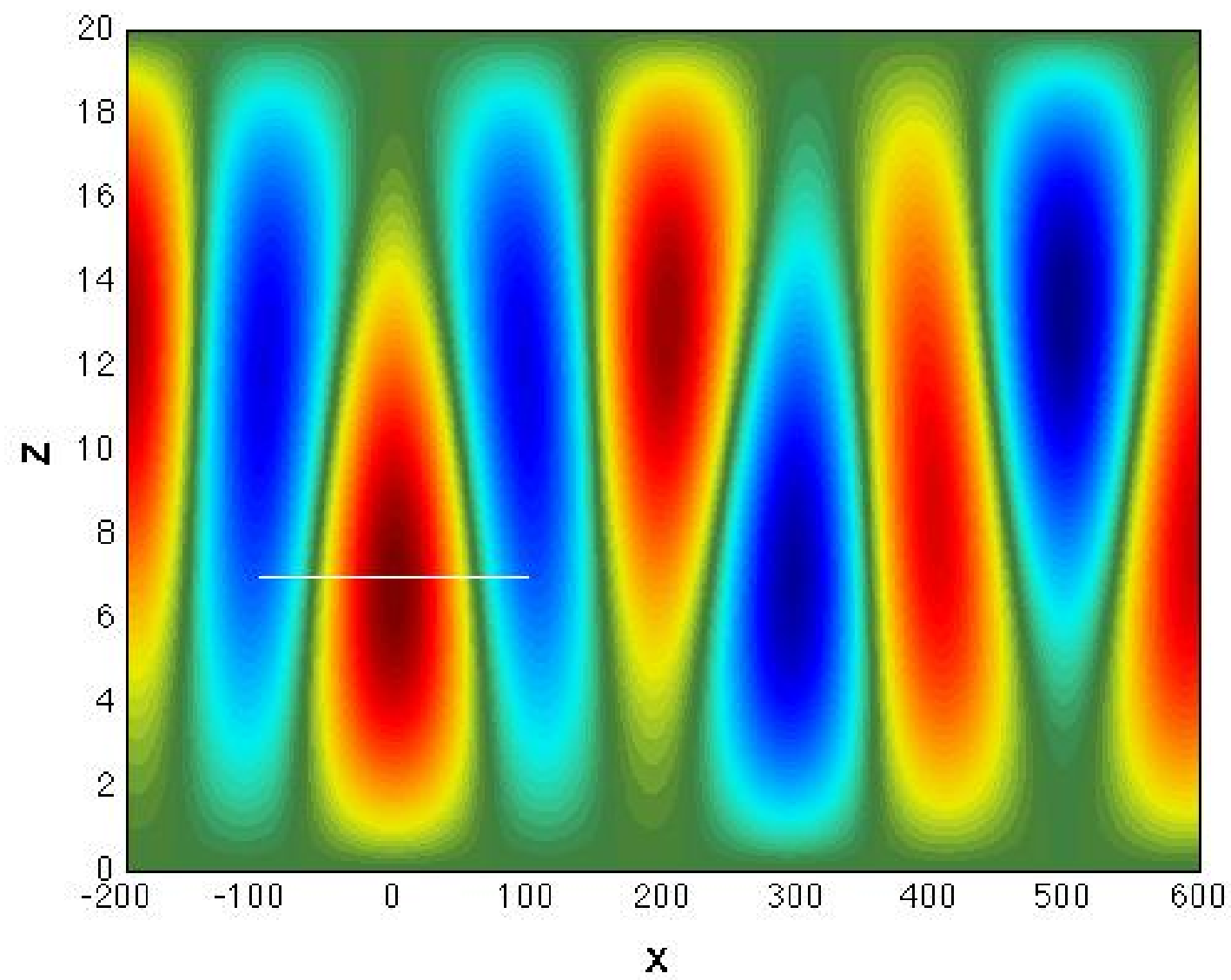

Figure 1.2: The stream function solution of the linear internal wave equation: the flow is along lines of constant color where the red gyres are counterclockwise and the blue gyres are clockwise. The whole map moves slowly from left to right. The initial position of the plankton is shown as a white line. 


\subsection{Model Initialization}

Many zooplankton follow a diel migration and have a daylight preferred light level [34] ${ }^{2}$. For example Euphausia pacifica are observed in thin layers during daylight hours at depths ranging from 60 to 95 meters ([25] figure 2). Since the daily migration and tidal forces are on different periods, there are a range of possible depths at which a thin plankton layer may encounter shear generated by tidal flow over topography. We may therefore choose the preferred light level of the plankton within our horizontal strip to display the mechanisms outlined below. For the chosen background flow we found that setting that $z_{L}=7$ meters allowed strong interaction of the populations with the high shear field.

Since zooplankton are observed in nepheloid layers, to initialize the simulation, an ensemble, $(N=10000)$ of plankton is introduced into the channel at $z_{L}$. These zooplankton swim at a fixed speed of $v_{\text {swim }}=0.1 \mathrm{~m} / \mathrm{s}$ according to the models developed and described in section 2. This value for $v_{\text {swim }}$ is on the high end of the observed values in table 2 of [12], and matches that used by [25]. The simulations model approximately 67 minutes of real world time, over which the values of both $z_{L}$ and the flow parameters may be assumed to be near constant. The boundary conditions for the plankton at the impermeable barriers at $z=H$ and $z=0$ are reflective. This choice does not really affect the fluid particle model, but it does make the Stokes' inertia model described in 2 less physical very close to the boundary because there is an instantaneous change of direction in $z$. However, since we are using the Euler equations with free-slip conditions, the flow near the boundary is unphysical in itself, and we will accept the simplified near boundary behavior for now. In the future, when we move to the full Navier-Stokes equations, we can revisit these conditions.

\footnotetext{
${ }^{2}$ The adaptive significance of this is thought to be the avoidance of predators during daylight hours [4]
} 


\section{Chapter 2}

\section{Modeling the Motion of Plankton}

This research was inspired primarily by [25], in which several responses of plankton to shear were modelled and the results compared to acoustic data from Knight Inlet in British Columbia, Canada. In the simulations in that paper, encountering the bottom boundary layer triggered the shear response. It is true that shear is created near the bottom boundary, but shear could occur anywhere else in the fluid as well. We wish to build a plankton motion model which will account for this fact. This gives us the following list of features that are important for a descriptive model of the motion of zooplankton.

\section{Accurate Physics}

2. Swimming

\section{A Shear Response}

We will address each in turn.

\subsection{Particle Models:}

\subsubsection{An Overview of Particle Models:}

First, we need to model the motion of plankton as they are advected by seawater. Zooplankton are small, on the order of a centimeter [25]. Due to the discrepancy in size with 
flow features we will consider them as point mass particles. The dynamics of two-way models (in which the particles actively modify the flow) are necessarily more complicated due to the coupling of the effects. For example, Wallner and Meiburg [60] found that using a two-way model led to differences in vorticity at the center of gyres in particle laden mixing layers. The importance of two-way coupling is quantified in [9] by considering the Stokes numbers of the particles as well as the ratio of their aggregate volume to the volume of the fluid in which they are suspended. In our exploration of parameter space, we find that across all models, before they are stochastically perturbed by swimming, our Stokes numbers remain less than one, so that particles follow the flow closely. Our volume ratios remain small as well, so that according to Crowe et al's [9] criterion we are well-justified in employing one-way models. While two-way models are, in principle, more accurate than one-way models because they take into account both effects, for particles of small mass and size the assumption that the presence of the particles does not affect the motion of the fluid is a physically plausible approximation. This is the "passive tracer" assumption, and following the literature we will assume its validity in all of our models.

We will focus on models for which it is assumed the particles in question are spherical. The first reason is because this is simpler. The second is that the natural case to try after spherical is ellipsoid, and it is not clear that the population distribution would be greatly affected by this change. For instance, see [24] for an analysis where they concluded that for nearly stable organisms the effects of making the particles elliptic could be ignored for the case of Kelvin Helmholtz billows. Euphausia pacifica are nearly stable organisms according to the formulae presented in [24], and Kelvin Helmholtz billows are a significant event for a population to pass through, so we have doubts that a choice of elliptical particles would make much difference in the internal wave induced flows that we consider. Any number of more complicated geometries may be considered in the future should those effects prove important. While the hydrodynamics of the propulsion of individual plankters is interesting (see for example [42]), we are interested in population dynamics and are satisfied with this approximation. Under the passive tracer and spherical particle assumptions we can consider the hierarchy depicted in Figure 2.1. 


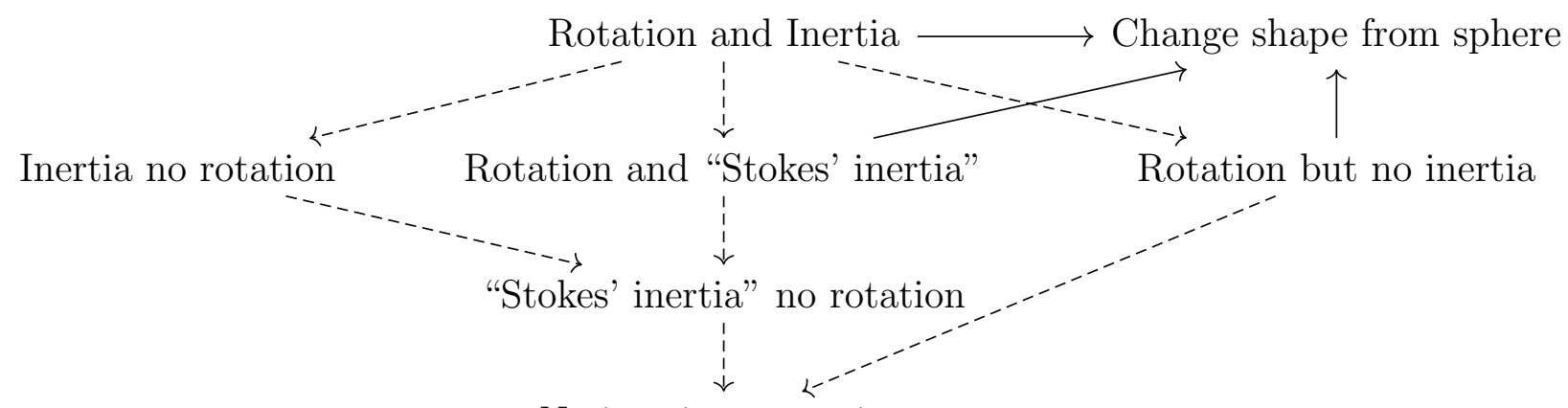

No inertia or rotation

Figure 2.1: A hierarchy of possible particle models. Here the dotted arrows mean "simplify" and the solid arrows mean "complicate."

By "Stokes' inertia" we mean the inertial particle model we will develop in section 2.1.4, a simplified form of the Maxey-Riley equations. A model which includes all effects of both rotation and inertia is the most complicated model in this hierarchy. If we wish to simplify it we have several layers of simplifying choices, which produce the above hierarchy.

Models including the effects of torques, and therefore rotations, are called gyrotactic models. Many species of phytoplankton swim up and are bottom heavy [15]. In such cases shear flows can rotate the plankton so that their ascent is halted, and they form a layer. This case was examined in both [24] and [15] and elsewear. While interesting and explanatory for these single celled organisms, rotation by shear is only one way that zooplankton may be affected by shear.

The Kolmogorov length is the scale at which mechanical energy is dissipated into heat. Ianson et al. [25] give estimates of the dissipation of turbulence kinetic energy in Knight Inlet, and these values show that the Kolmogorov length scale is longer than the phytoplankton by an order of magnitude, but shorter than the zooplankton by about two orders of magnitude ([15], [25]). Therefore viscosity dominates in the regime where phytoplankton live, but not in the regime where zooplankton live. This means that zooplankton may be affected by more turbulent flows and shear at much longer length scales. These conditions may not only rotate their bodies to confound their swimming, but also compress or shear their bodies. We expect flows which affect them in any of these ways to be high shear. Therefore by building in a more general shear response we are incorporating a response 
to more than just reorientation. For this reason we will not study gyrotactic models for plankton response to shear, which have been studied in, for example, [15], [14], [24].

\subsubsection{The Fluid Particle Model}

At the bottom of the hierarchy in Figure 2.1 we have no inertia or rotation, which is the first model we consider. Fluid particles follow integral curves of the flow exactly, which means they change their motion instantaneously to match the flow. In this sense they have no set orientation or inertia. We refer to this model as "the fluid particle model". Mathematically, for a flow $(u, w)$ in two dimensions we have the position $(x, z)$ of a fluid particle given by

$$
\begin{aligned}
& \dot{x}=u(x, z, t) \\
& \dot{z}=w(x, z, t)
\end{aligned}
$$

The fluid particle model for the motion of plankton is simply to transport the plankton as though they were a fluid particle.

While mathematically convenient, the following argument shows that the fluid particle model is too simple because it ignores both inertia and buoyancy. In [54], the parameter $\alpha$ is introduced to measure the importance of viscous effects compared to inertial effects. For Euphausia pacifica, using the value for the density in [22] we obtain,

$$
\alpha \equiv \frac{6 \pi a \mu}{m_{p}+\frac{1}{2} m_{f}}=\frac{6 \pi \cdot 0.004 \cdot 10^{-3}}{\frac{4}{3} \pi 0.004^{3} \cdot 1063+\frac{2}{3} \pi 0.004^{3} 1027} \approx 0.1784
$$

so that inertia is important. The parameter $\beta$ measures the importance of gravitational effects compared to viscous effects.

$$
\beta \equiv \frac{\left(m_{p}-m_{f}\right) g}{6 \pi a \mu}=\frac{\left(\frac{4}{3} \pi 0.004^{3} \cdot 1063-\frac{4}{3} \pi 0.004^{3} 1027\right) 9.81}{6 \pi \cdot 0.004 \cdot 10^{-3}} \approx 1.256
$$

so gravity is important. By extension then, the relative importance of gravity to inertia is then given by 


$$
\alpha \beta \approx 0.224
$$

which means that inertial effects are important compared to gravitational effects. Therefore inertia should be considered. As we will see, the method with which we model inertia will also include a term modelling the buoyancy.

\subsubsection{A Particle Model with Inertia}

For a general inertial particle of mass $m_{p}=\frac{4}{3} \pi a^{3} \rho_{p}$, the velocity $\mathbf{v}$ is given by Newton's second law and we get the system

$$
\begin{aligned}
m_{p} \dot{\mathbf{v}} & =\mathbf{F}_{\text {undisturbed flow }}+\mathbf{F}_{\text {buoyancy }}+\mathbf{F}_{\text {Stokes' drag }}+\mathbf{F}_{\text {added mass }} \\
& +\mathbf{F}_{\text {viscous memory }}+\mathbf{F}_{\text {vorticity induced lift }}+\mathbf{F}_{\text {propulsion }} \\
\dot{\mathbf{x}} & =\mathbf{v}
\end{aligned}
$$

where $\mathbf{F}_{\text {propulsion }}$ is the force caused by the swimming of the particle. The list of forces on the right hand side are approximated by the Maxey-Riley equations [7]:

$$
\begin{aligned}
m_{p} \dot{\mathbf{v}} & =m_{f} \frac{D}{D t} \mathbf{u}-\frac{1}{2} m_{f}\left(\dot{\mathbf{v}}-\frac{D}{D t}\left[\mathbf{u}+\frac{1}{10} a^{2} \nabla^{2} \mathbf{u}\right]\right)-6 \pi a \rho_{f} \nu\left(\mathbf{v}-\mathbf{u}-\frac{1}{6} a^{2} \nabla^{2} \mathbf{u}\right) \\
& +\left(m_{p}-m_{f}\right) \mathbf{g}-6 \pi a^{2} \rho_{f} \nu \int_{0}^{t} \frac{\partial}{\partial \tau}\left(\mathbf{v}-\mathbf{u}-\frac{1}{6} a^{2} \nabla^{2} \mathbf{u}\right) \frac{d \tau}{\sqrt{\pi \nu(t-\tau)}}
\end{aligned}
$$

where the values of $\mathbf{u}$ are taken at the position of the particle, $\mathbf{g}$ is the force of gravity, $m_{f}$ is the mass of the fluid displaced by the particle, $\rho_{f}$ is the density of the fluid, $\nu$ is the kinematic viscosity of the water, and $a$ is the radius of the spherical particle. The terms in this equation correspond to the forces above except for the propulsion of the particle, 
which we return to in section 2.2. In non-dimensional form we have [51]

$$
\begin{aligned}
\mathbf{F}_{\text {undisturbed fluid flow }} & \leftrightarrow+\frac{3 R}{2} \frac{D \mathbf{u}}{D t} \\
\mathbf{F}_{\text {buoyancy }} & \leftrightarrow+\left(1-\frac{3 R}{2}\right) \mathbf{g} \\
\mathbf{F}_{\text {Stokes' drag }} & \leftrightarrow-\frac{R}{S t}\left(\mathbf{v}-\mathbf{u}-\frac{a^{2}}{6} \nabla^{2} \mathbf{u}\right) \\
\mathbf{F}_{\text {added mass }} & \leftrightarrow+\frac{R a^{2}}{20} \frac{D}{D t} \nabla^{2} \mathbf{u} \\
\mathbf{F}_{\text {viscous memory }} & \leftrightarrow-R \sqrt{\frac{9}{2 \pi S t}} \int_{0}^{t} \frac{1}{\sqrt{t-s}}\left(\dot{\mathbf{v}}(s)-\frac{d}{d s}\left(\mathbf{u}+\frac{a^{2}}{6} \nabla^{2} \mathbf{u}\right)\right) d s \\
\mathbf{F}_{\text {vorticity induced lift }} & \leftrightarrow+\frac{6.46 R}{\frac{4}{3} \pi a} \sqrt{\frac{1}{R e|\omega|}}(\mathbf{v}-\mathbf{u}) \times \omega
\end{aligned}
$$

Where the mass ratio parameter $R=\frac{m_{f}}{m_{p}+\frac{1}{2} m_{f}} \in(0,2)$ captures the range of dynamics caused by differing densities and $S t=\frac{2}{9} a^{2} \Re$ is a Stokes number. Now we've accounted for all the forces except those caused by the propulsion of the particle. A few ways of adding noise to the Maxey-Riley equations are explored in [51]. The authors drive an electrolytic fluid electromagnetically and find that their tracers display stochastic fluctuations not predicted by Maxey-Riley. In an attempt to model the observed fluctuations they try simplified Maxey-Riley models with additive and multiplicative white noise, as well as with additive coloured noise. After some analysis they select the additive colored noise version as the most effective stochastic model to match experimental results. While interesting, this type of stochastic Maxey-Riley model requires coefficients that must be determined experimentally, which is of no help to those of us who do not have their experimental apparatus. Additionally, we wish to focus on swimming, which is not directly related to their problem. We will also find that the introduction of noise in all terms is unnecessary, because the physical assumptions of our model will let us drop some of the terms altogether. For this reason we will return to the propulsion force effects at the end of the justification of our model. 


\subsubsection{The Stokes' Inertia Model}

The Maxey-Riley equations are complex enough to be numerically expensive to simulate (especially the memory term, sometimes referred to as the Basset term), so we make some reasonable approximations. In this paper we are considering Euphausia pacifica as a concrete example. The individuals in this zooplankton species measure one to two centimeters in length [25]. The memory integral term accounts for the effects of viscosity on the particle in the past that are relevant to its current motion. The low kinematic viscosity of seawater gives us a high Reynolds number. This fact combined with the small particle radius, allows us to omit the memory integral as an approximation. Since we are working with very long waves and a small particle radius we can omit the terms involving $a^{2} \nabla^{2} \mathbf{u}$, which means we simplify both the added mass and Stokes drag forces. A full discussion of the terms in the Maxey-Riley equations can be found in [7], and this reference provides more detailed justification for the claims made in this paragraph. Dropping the terms just described, the Maxey-Riley equations become

$$
\dot{\mathbf{v}}=\frac{1}{m_{p}+\frac{1}{2} m_{f}} \cdot\left(\frac{3}{2} m_{f} \frac{D \mathbf{u}}{D t}-6 \pi a \rho_{f} \nu(\mathbf{v}-\mathbf{u})+\left(m_{p}-m_{f}\right) \mathbf{g}\right)
$$

Now we have the system

$$
\begin{aligned}
\dot{\mathbf{v}} & =\frac{1}{m_{p}+\frac{1}{2} m_{f}} \cdot\left(\frac{3}{2} m_{f} \frac{D \mathbf{u}}{D t}-6 \pi a \rho_{f} \nu(\mathbf{v}-\mathbf{u})+\left(m_{p}-m_{f}\right) \mathbf{g}\right) \\
\dot{\mathbf{x}} & =\mathbf{v}
\end{aligned}
$$

The integral curves of this equation are approximate time evolution paths for particles with inertia advected by a background flow. The only term that is left to model inertia, besides the gravity term, is the Stokes' drag term. This is why we refer to this model as "Stokes' inertia." Stokes drag is valid in the low Reynolds number limit [31], so we are taking this as an approximation (see 2.3.2 for a discussion). We have thus moved up one rung in the hierarchy depicted in 2.1.1. Equations similar to this simplified Maxey-Riley equation have been studied in [23], [57], and [3] in different contexts. Consider the dynamics described by these equations. Suppose $\mathbf{u}$ is constant, and a neutrally buoyant particle is released into the flow from rest, so that $\mathbf{v}(0)=\mathbf{0}$. Since the flow is constant and the particle is neutrally buoyant, equation 2.1 simplifies to

$$
\dot{\mathbf{v}}=\frac{-6 \pi a \rho_{f} \nu(\mathbf{v}-\mathbf{u})}{m_{p}+\frac{1}{2} m_{f}}=-C(\mathbf{v}-\mathbf{u})
$$


for a positive constant $C$. With initial condition $\mathbf{v}=\mathbf{0}$ the solution is just

$$
\mathbf{v}=\mathbf{u}(1-\exp (-C t))
$$

so that the particle will accelerate in the direction of $\mathbf{u}$ until it approaches $\mathbf{v}=\mathbf{u}$, at which point the acceleration will be $\dot{\mathbf{v}} \approx \mathbf{0}$. Let's consider a few limiting cases for

$$
C=\frac{6 \pi \rho_{f} a \nu}{m_{p}+\frac{1}{2} m_{f}}
$$

as $\rho_{p}$ changes. Notice that this means $m_{p}=\frac{4}{3} \pi \rho_{p} a^{3}$ will change as well. We have

$$
\begin{aligned}
& \lim _{\rho_{p} \rightarrow \infty} C=0 \\
& \lim _{\rho_{p} \rightarrow 0} C=\frac{6 \pi \rho_{f} a \nu}{\frac{1}{2} \frac{4}{3} \pi a^{3} \rho_{f}}=\frac{9 \nu}{a^{2}}
\end{aligned}
$$

From the first limit we have that a particle of infinite mass will not be accelerated, as expected. The more useful idea is that following $\mathbf{F}=m \dot{\mathbf{v}}$, the more massive the particle, the less acceleration it will experience for a given constant force. For the second limit we see that for particles of small mass it is the ratio of viscosity to radius squared that matters. The acceleration is proportional to the viscosity, and inversely proportional to the surface area. This means that more viscous fluids will accelerate a particle to agree with the fluid velocity faster than less viscous fluids, and that smaller particles will be accelerated to agree with the fluid velocity faster than larger particles. All of this confirms our intuition of what an equation which captures inertia should do: gradually accelerate a particle to agree with the fluid velocity, with that acceleration depending on the mass, viscosity, and size. An example is shown in Figure 2.2. We note that we need not start the particle at rest: the particle will be accelerated over time to agree with the fluid flow regardless of initial state. If gravity is included then the difference in mass is important. In this case the particle is accelerated to agree with a combination of the fluid flow and gravitational forces. 


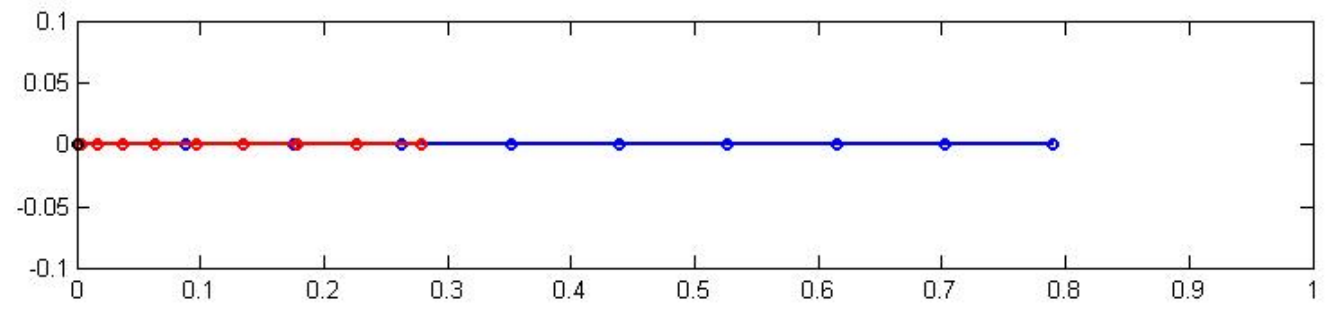

Figure 2.2: The paths followed by a fluid particle (in blue) and a particle with inertia (in red), released from the origin. The inertial particle is initially at rest, and is gradually accelerated to match the speed of the flow $\mathbf{u}=(0.1,0)$. The fluid particle matches the flow exactly.

Now consider the case where $\mathbf{u}$ is not constant. Take a small time interval $\Delta t$, and approximate the value of $\mathbf{u}$ at the particle's location by a constant vector over $\Delta t$. We can then apply the same analysis as in the constant case in each short time interval. If $\Delta t$ is made smaller than the time step in our simulation that is sufficient. In particular, for sufficiently smooth $\mathbf{u}$, and sufficiently small $\Delta t$, we can approximate the value of the vector field over the time interval by its value at one of the endpoints. The point is that the same analysis holds: the particle will be accelerated over time to agree with the fluid flow and if the fluid flow is changing, so is the acceleration. This can be seen in Figure 2.3. Once again if the particle is not neutrally buoyant then the particle is accelerated by a combination of the gravity and fluid forces during each short time interval. 


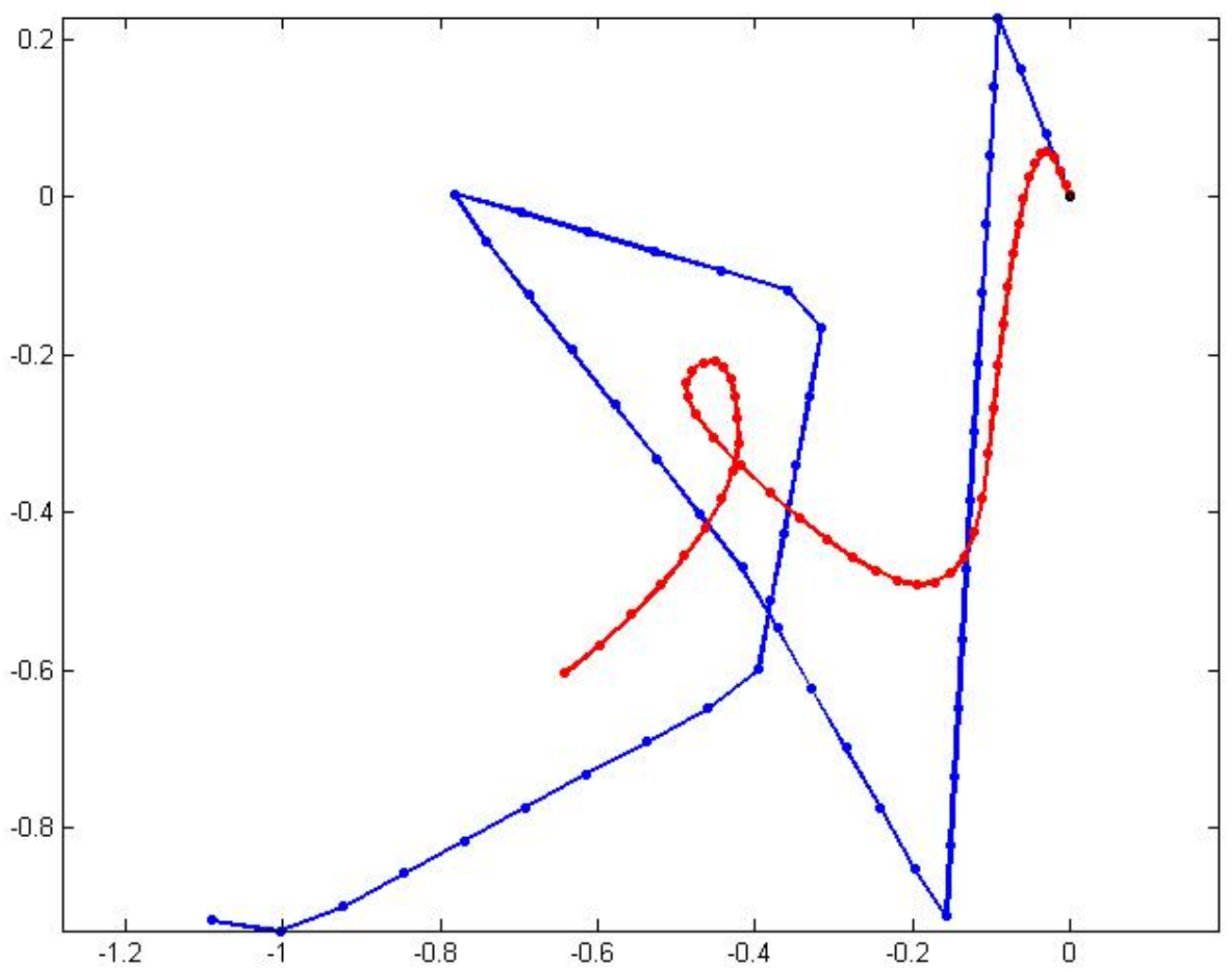

Figure 2.3: The trajectory of an inertialess particle in blue matches a randomly changing u. In red, a particle with inertia initially at rest at the origin is gradually accelerated to match the speed of the flow, but with every change of the flow's direction we get a new acceleration. For this simulation we took the flow's magnitude to be constant, with randomly changing direction. It is clear that the inertia acts to smooth the particle path.

\subsubsection{The Effects of Relative Density}

The difference between the models with inertia and those without depends on the density of the particle. As mentioned, the mass ratio parameter $R=\frac{m_{f}}{m_{p}+\frac{1}{2} m_{f}} \in(0,2)$ captures the 
range of dynamics caused by differing densities [7]. If particles have higher density than the fluid $R<\frac{2}{3}$, and they are referred to as 'aerosols.' If particles have lower density than the fluid $R>\frac{2}{3}$, and they are referred to as 'bubbles.' When gravity is included aerosols sink and bubble float. Using a perturbation analysis, Maxey [40] was able to show that aerosols tend to move away from regions of high vorticity and bubbles tend to move to the center of regions of high vorticity. Intuitively we can think of these effects as the result of centrifugal forces acting on the particle.

It should also be noted that although [7] and [40] characterize the effects of changing $R$ in terms of the intensity of the vorticity, there is more subtlety here. The particles are not attracted to or repelled by regions of high vorticity as the following examples show. First, consider the shear flow $\left(z^{2}, 0\right)$, which has vorticity $2 z$, a linear dependence on $z$. A bubble accelerated from rest by this flow (without gravity included) will be pushed to the right, but if it seeks high vorticity regions it must also move upward, to higher vorticity, and there is no mechanism to do so. So bubbles do not seek regions of high vorticity.

Now consider the example of an irrotational vortex, which has no vorticity. An aerosol will still be pushed out of such a vortex by inertia. Mathematically we can see that the term $\frac{3 R}{2} \frac{D \mathbf{u}}{D t}$ controls the acceleration of the particle by the background fluid. For bubbles with $m_{p}<<m_{f}, \frac{3 R}{2} \approx 3$, so that the acceleration is about 3 times higher than that experienced by a fluid particle. Since the acceleration in a gyre is towards the center, bubbles congregate there. Likewise for aerosols with $m_{p}>>m_{f}, \frac{3 R}{2} \approx 0$ and there is very little acceleration by the fluid, as expected, so particles leave regions of high vorticity. However, as our counterexamples show, it is not vorticity that is the mechanism, but the acceleration direction which causes these effects, and in particular the component of acceleration normal to the pathline. Therefore these 'vorticity preference effects' are actually the result of the interaction of inertia with the curvature of the pathlines. It does seem plausible that generally high acceleration and pathline curvature occur with intense vorticity, but a thorough investigation of this relationship is beyond the scope of the current investigations. We will follow the literature and characterize the effects of changing $R$ as 'vorticity preference effects', but the subtleties outlined here should not be forgotten.

In the neutrally buoyant case, $m_{f}=m_{p}, R=\frac{2}{3}$, and there are no gravitational or vorticity preference effects. This choice also ensures that the acceleration by the background flow $\frac{3 R}{2} \frac{D \mathbf{u}}{D t}$ reduces to $\frac{D \mathbf{u}}{D t}$, which is the same acceleration experienced by a fluid particle. Although neutrally buoyant particles experience the same same acceleration by the background flow, neutrally buoyant particles still do not follow the integral curves described by 
fluid particles because they have nonzero inertia. This is reflected in our models through the effects of drag and gravity. We will refer to the Stokes' inertia model with $R=\frac{2}{3}$ as the "neutral buoyancy Stokes' inertia" model. There is a good review of the neutrally buoyant case in [7], but the relavant points can be summarized once we define a few more quantities. Toward this, the Okubo-Weiss parameter is given by

$$
Q=-\operatorname{det}\left(\begin{array}{cc}
u_{x} & u_{z} \\
w_{x} & w_{z}
\end{array}\right)
$$

It turns out that when $Q>0$ deformation dominates, as it does around hyperbolic stagnation points, and if $Q<0$ rotation dominates, as around elliptic stagnation points. We also define the Stokes number ${ }^{1}$

$$
S t=\frac{U\left(m_{p}+\frac{1}{2} m_{f}\right)}{6 \pi a \rho_{f} \nu L}
$$

where $U$ is a characteristic speed of the flow, and $L$ is a characteristic lengthscale of the flow. The Stokes number represents the dimensionless decay time of the particle velocity, so that with large $S t$, the particle has less dependence on the fluid flow. Notice that $S t=\frac{U}{L C}$ where $C$ is the constant from equation 2.2 of section 2.1.4 which controls the time it takes a particle to agree with the background flow. Since $\frac{U}{L}$ is fixed, we have the following correspondences

$$
\begin{aligned}
& \text { large } S t \leftrightarrow \text { small } C \leftrightarrow \text { gradual agreement with the flow } \\
& \text { small } S t \leftrightarrow \text { large } C \leftrightarrow \text { rapid agreement with the flow }
\end{aligned}
$$

For neutrally buoyant particles near a hyperbolic stagnation point $Q>0$, so that deformation dominates. If $Q>\frac{1}{S t^{2}}$, particle and flow trajectories separate exponentially [7]. Over the range of flows in our simulations $Q<<\frac{1}{S t^{2}}$ and we cannot conclude that particle and flow trajectories separate exponentially, and indeed from observation we have no indication that this is the case. This makes sense since the Stokes number remains small so we would expect particles to quickly follow the flow. Over the parameter space we tested the main difference in the neutrally buoyant Stokes' inertia model from the fluid particle model was that the drag causes the particle trajectories to lag behind where they would be in the fluid particle model. Neutrally buoyant particles in our model follow integral curves closely for slow fluid speeds, but this 'tracking' approximation gets worse as fluid speed is increased.

\footnotetext{
${ }^{1}$ some authors would call this $\frac{S t}{R}$, but we will not.
} 
For the slower fluid speeds, over a range of mass, (and so $R$ ) values, the vorticity preference effects were hardly noticeable because the vorticity was small. In future work we will consider turbulent flows in the coastal ocean which can have regions of high vorticity on a scale comparable to the size of Euphausia pacifica. In the current work we consider linear superpositions of waves having regions of vorticity orders of magnitude larger than the size of the particles in question. We expect these vorticity preference effects to be negligible, even when gravity is not included. When $R \neq \frac{2}{3}$ and gravity is included, it can play a very important role in the dynamics. For sufficiently dense particles the tendency to leave regions of high vorticity is a relatively small factor compared to the acceleration due to gravity. In fact for the range of flow speeds we considered, even for particles with a $1 \%$ density difference from the fluid, corresponding to $R=0.6623<\frac{2}{3}$ and $R=0.6711>\frac{2}{3}$, the dominance of gravity is immediate and the more subtle vorticity preferences of the particles are imperceptible.

One may object, noting that inertia was deemed more important than gravity in the scaling arguments presented in section 2.1.2. However those arguments are for flows in general, meaning they attempt to make a statement about all flows. Our flows have no small scale structure, which we believe goes a long way to explaining why the effects of inertia were so small in our simulations. So while the scaling arguments demand that we consider inertia, the specific flow in question seems to be an exception to the predictions made by those arguments. In future work we may consider realistic flow over topography, in which case there may be a great deal of small scale structure, and inertia may once again become important even at the level of populations.

For $R \approx \frac{2}{3}$, the effects of gravity are small and the vorticity preference effects are very small. In this case the Stokes' inertia and neutral buoyancy Stokes' inertia models are very similar on short timescales to the fluid particle model, but over longer time scales even slight gravitational effects cause a very different evolution. When $R \not \approx \frac{2}{3}$, the Stokes' inertia model diverges significantly from the other two models due to gravitational effects that overshadow vorticity preference effects. Flows with different relative values of $Q$ and $S t$ could display different dynamics, but for the values in our simulations this paragraph summarizes the dynamics well. This brings our exploration of the possible particle models to a close. For now, we are satisfied with examining the first two rungs on the hierarchy of 2.1.1. 


\subsection{Swimming:}

\subsubsection{The (Discrete) Run and Tumble Model}

The second step in developing our model is to add swimming. The Lagrangian particle model was constructed to emulate the in situ measurements of Euphausia pacifica provided in [12]. During the day, when the layer is stationary at the preferred light level, Figure 8 of [12] shows a roughly uniform distribution of swimming direction in a vertical cross-section. We therefore adopted the discrete "run and tumble" swimming model [58].

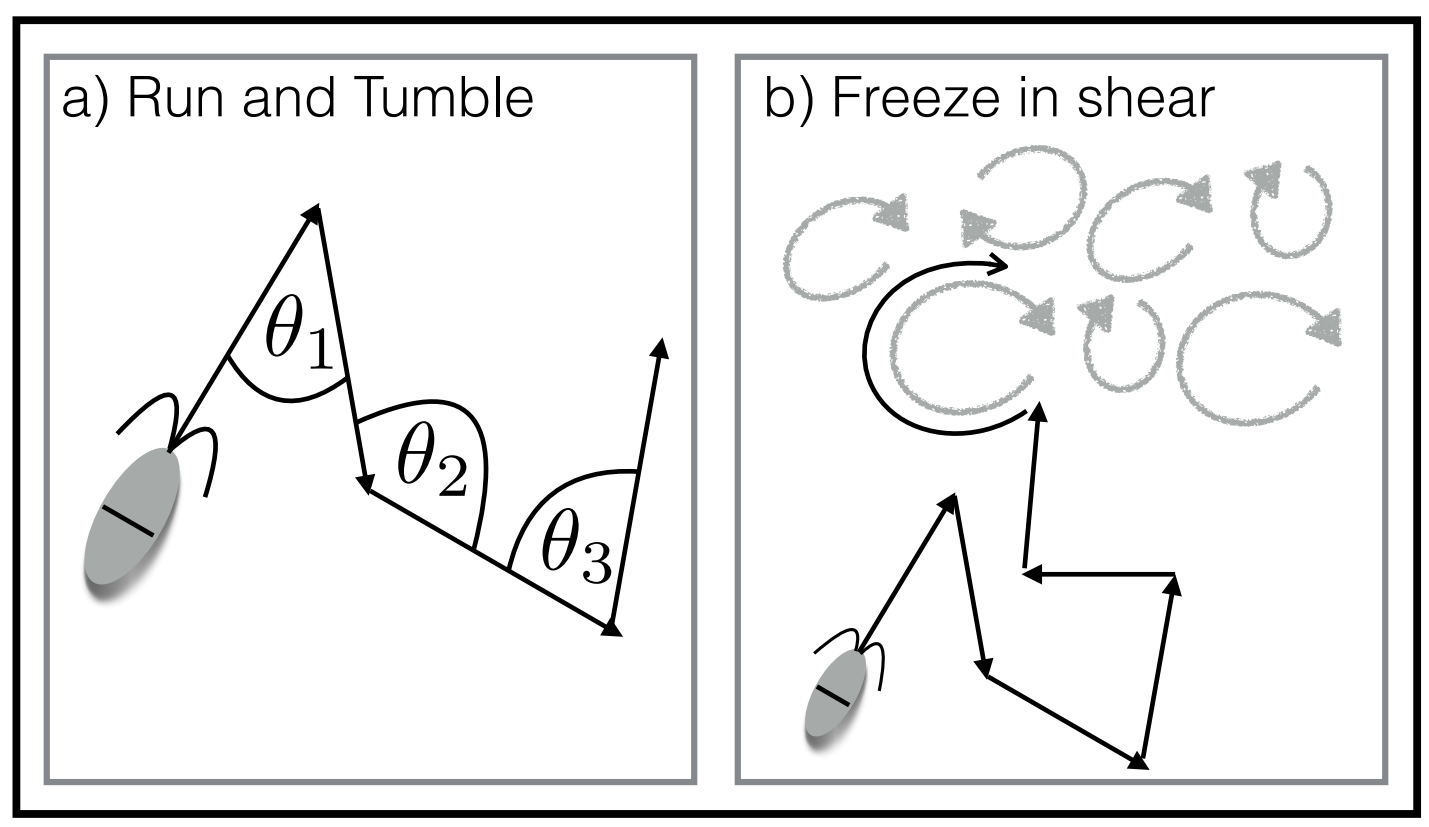

Figure 2.4: a) The discrete run and tumble model of plankton swimming. b) A variant of run and tumble where the plankton do not swim if the shear is too high. 
This model describes the swimming of plankton as a sequence of straight-line trajectories interrupted by instantaneous, uniformly random changes in direction, as illustrated in Figure 2.4 a). The plankton "run" a certain duration and then "tumble" to change their trajectory. The following assumptions are also made:

1. Decisions to change direction while swimming follow a Poisson process, so that the times between tumbles follow an exponential distribution.

2. The speed of the plankton while swimming is a constant $v_{s}$.

3. Each plankter moves independently of all others. The plankton do not interact.

The assumptions of discrete run and tumble were originally made in order to facilitate a classical Fokker-Planck analysis, rather than for their realism. One might ask why we did not adopt a more realistic swimming model, or even one of the other versions of run and tumble which lead to more physical trajectories. First, the discrete run and tumble model is easy to understand, code and modify, but there are more important reasons.

One reason to use this model is that the inclusion of inertia already leads to physically plausible trajectories. The term 'discrete' refers to the fact that the runs are uncorrelated. There is also a continuous model discussed in [58] where runs are correlated, leading to trajectories with a more consistent direction. While this makes the trajectory of an individual plankter more physically plausible, as we saw in 2.1.4 and 2.3, the addition of inertia also smooths out jagged paths, leading to physically plausible trajectories. Correlated runs would mean that a plankter would tend to head in the direction they were already headed but that is exactly inertia's effect. In light of this, it is our contention that the additional computational cost of correlating runs is not worth the gain in realism.

Another reason for this choice is that we are interested in the population dynamics in aggregate, rather than the modeling of individual plankton trajectories for their own sake. No matter how a correlated run was initialized, this effect would diminish over time, so that in large ensembles, and for times exceeding a few correlation periods, the effect of run correlations on the overall population dynamics are expected to be vanishingly small, especially when inertia is included.

For all these reasons we select the discrete run and tumble model (henceforth simply run and tumble), as the swimming component of the model. Mathematically we have 
$\left(u_{s}, w_{s}\right)=v_{\text {swim }}(\cos (\theta(t)), \sin (\theta(t)))$ where $\theta(t)$ is drawn from a uniform distribution over $(0,2 \pi)$.

\subsubsection{The Fluid Particle Model with Run and Tumble}

Adding swimming to the fluid particle is completely straightforward. Mathematically we have

$$
\begin{aligned}
\dot{x} & =u(x, z, t)+u_{s} \\
\dot{z} & =w(x, z, t)+w_{s}
\end{aligned}
$$

where the swimming in run and tumble is given in vector form by $\left(u_{s}, w_{s}\right)$ and the governing ODEs are considered to hold over the segments of time in which swimming occurs, separated by instantaneous changes in direction. All we have done is added the swimming to the definition of an integral curve. Physically, these equations tell us that the velocity of the particle is given by the velocity of the seawater plus the velocity of the swimming. The particles do not follow the same integral curve throughout the simulation because their swimming causes them to change which curve they are following. This coupling of two very simple models has led us to a model which is much more difficult to analyze than either one on its own. However, this is completely straightforward to simulate and computationally inexpensive. The effect of adding stochastic swimming is to make the paths continuous but only piecewise smooth.

\subsubsection{Stokes' Inertia with Run and Tumble}

We need to add swimming to the Stokes' inertia model as well, which corresponds to accounting for $\mathbf{F}_{\text {propulsion }}$ in the force balance equation. Again, we will adopt the run and tumble model. As mentioned, in this model each plankter swims at a uniform speed so that in between turning events the swimming is a constant vector. Therefore we can apply the same analysis used when there was no swimming by considering the swimming to be part of the background flow. Instead of constant $\mathbf{u}$, the constant background is $\mathbf{s}+\mathbf{u}$, where $\mathbf{s}$ is the swimming vector. This constant background changes every small timestep $\Delta t$. That is, we are stochastically perturbing the flow by the swimming, and using the result as our 
flow. In light of all the arguments, we now define

$$
\begin{aligned}
\dot{\mathbf{v}} & =\frac{1}{m_{p}+\frac{1}{2} m_{f}} \cdot\left(\frac{3}{2} m_{f} \frac{D \mathbf{u}}{D t}-6 \pi a \rho_{f} \nu(\mathbf{v}-(\mathbf{s}+\mathbf{u}))+\left(m_{p}-m_{f}\right) \mathbf{g}\right) \\
\dot{\mathbf{x}} & =\mathbf{v}
\end{aligned}
$$

as the evolution equations for Stokes' inertia with run and tumble. Note we did not add swimming to the material derivative because it is piecewise constant and so has either a zero or undefined derivative. When this equation is discretized it will always be defined, and will be zero. Physically, this is an appeal to the passive tracer assumption: since the swimming of the plankton does not affect the flow, it should not affect the force on the plankton by the undisturbed flow either. Note that this has also added swimming to the neutral buoyancy Stokes' inertia model since that model corresponds to the choice of $m_{p}=m_{f}$.

\subsubsection{The Effects of Density under Run and Tumble}

Now that we have added swimming to all three models, it is natural to ask what the differences between them are. As when we compared these two models without swimming in section 2.1.5, we first consider the zero gravity version of the Stokes' inertia model. Run and tumble is an isotropic diffusion process so if the water is motionless the plankton should approach a uniform distribution in the domain. The Stokes' inertia model includes both vorticity preference effects and swimming. Aerosols tend to move away from regions of high vorticity anyway, and will now be more likely to do so as they swim according to a diffusion process. Bubbles, on the other hand, can reach a balance where their tendency to seek out the center of vortices is counteracted by the tendency of swimming to increase variance. The vorticity preference effects are the result of consistent acceleration towards, or away from, the center of the vortex. This consistency is interrupted by the random nature of the swimming, but can be overcome by sufficiently strong vortices, or sufficiently extreme values of $R$. Clearly the strength of the vorticity preference effects will depend on some balance of vorticity, relative masses, the scale of the vorticity regions, and the swim speed, but exactly how these interact is beyond the scope of our current investigations.

Once gravity is included, for the flows tested, simulations show that the inclusion of an isotropic swimming component to the Stokes' inertia model, resulting in equation 2.3,

leads to gravity dominated dynamics for $R \approx \frac{2}{3}$. For $R \approx \frac{2}{3}$ the effects of gravity are small 
and the vorticity preference effects are very small, leaving the swimming and fluid drift to compete for dominance in the almost neutrally buoyant dynamics. Once again, in broad terms it seems that neutrally buoyant Stokes' inertia is well approximated by the fluid particle model with a slight lag time in following integral curves, and Stokes' inertia is well approximated by the neutral buoyancy Stokes inertia with an additional, small, evolution downward.

Without swimming, both the fluid particle and Stokes' inertia models are deterministic, and we can think in terms of integral curves the particles are on (or near). Swimming causes the particles to randomly change which integral curves they are on, or close to. The speed of the swimming is fixed, which limits these changes to integral curves which are nearby. Since the flow structures in our case are large, with gently curving streamlines, we expect swimming to not change the trajectory too much over short timescales. Of course as the process of swimming is repeated many times these small changes may make an enormous difference, and we cannot say what will happen to a given plankter, even approximately. As we saw in Figure 2.3, inertia acts as a smoother to the motion of the particle perturbed by the water around it. This creates a difference in the nature of the trajectories as we expected: the Stokes' inertia plankton trajectories are more physical than the relatively more jagged trajectories of the plankton from the fluid particle model. Whether this has an appreciable affect on the population dynamics remains to be seen.

\subsubsection{Stokes' Inertia with Run and Tumble: Our Specific Case}

Up until now, we have considered the dynamics of the equations 2.1 and 2.3 in general. Our model is flexible, but we will focus on the zooplankter Euphausia pacifica in order to provide a concrete example. These zooplankton are one to two centimeters in length and sink at a rate of $0.5 \mathrm{~cm} / \mathrm{s}$ in still water when not swimming [25]. It is not clear from the literature that this value is the actual sinking speed of Euphausia pacifica. However, this was the value used by [25] and it seems to be correct at least up to order of magnitude from observations. See for example [50], which is the paper they cite as justification for their value, in which the sinking speeds of many copepods are recorded, although not actually Euphausia pacifica. One of the contentions of [50] is that the downward migration portion of the diel cycle could be attributable entirely to passive sinking. If this is true then in situ

measurements such as those presented in [43] give some possible justification for taking this value, although measurements suggest the sinking speed may be faster than the value 
taken. Since we are following [25] for the other constants, we will follow them here as well.

Approximating a plankter as a sphere of the same mass gives a rough estimate of $a=0.004 \mathrm{~m}$. Using the Stokes' settling velocity formula [31], we have ${ }^{2}$

$$
\begin{aligned}
0.005 & =\frac{2}{9} \frac{\left(\rho_{p}-\rho_{f}\right)}{\mu} g \cdot a^{2}=\frac{2}{9} \frac{\left(\rho_{p}-1027\right)}{1.6 \cdot 10^{-3}} 9.81 \cdot 0.004^{2} \\
\rho_{p} & =1027.2293 \approx 1027.23 \frac{\mathrm{kg}}{\mathrm{m}^{3}}
\end{aligned}
$$

By construction then, this density value was chosen to make the dynamics physical. Picking a constant value for the density is necessary for the model but some zooplankton can regulate their buoyancy, which makes any value chosen an "effective density" rather than an actual one. There is a great deal of literature on plankton buoyancy regulation. To quote [30], "The mechanisms by which zooplankton regulate buoyancy (Bidigare and Biggs, 1980; Mills, 1984; Sanders and Childress, 1988), and the significance of specific internal structures, cf. the oil sac of C. Finmarhicus (Miller it al., 1988; Francis et al., 1999), should deserve particular attnetion with respect to mass density determinations and the animals' life history strategies." The density of Euphausiids change over their lifetime. See for example figure 2 of [48]. Due to the composition of their bodies, the buoyancy of plankton changes with depth [6]. They also argue that zooplankton must have some kind of buoyancy regulation mechanisms:

"Whether an animal floats or sinks depends on the density difference between it and the surrounding seawater. Thus, a neutrally buoyant animal must have the same aggregate density as the surrounding seawater. However, the greater compressibility of lipids compared to seawater means that any depth of neutral buoyancy will not be stable. In other words, below the depth of neutral buoyancy, lipid will become denser (as pressure increases), and thus the aggregate density of the animal will become greater as well. The converse is also true. Therefore, any displacement of the animal away from its depth of neutral buoyancy should result in it accelerating away from that depth. Hence, the presence of lipids is more than a barrier to downward migration (as suggested by Yayanos et al. 1978), or a means to promote upward migration (as suggested by Visser \&5 Jónasdóttir 1999); it actually represents an impediment to maintaining position in the water column. Moreover, as we will illustrate, the buoyancy properties of an animal are extremely sensitive to the relative composition of its biochemical constituents... the buoyancy properties of an individual are extremely sensitive to the relative biochemical composition (see above), and

\footnotetext{
${ }^{2}$ The value of $\mu$ here assumes the water is around 10 degrees Celsius.
} 
biochemical composition does change. In the example presented here, a change of only a few percent makes a tremendous difference on the buoyancy properties of the animal (Fig. 1). It is therefore not unreasonable to expect large changes in the buoyancy properties of individuals as they grow, mature and reproduce. It is also not unreasonable to expect them to possess a buoyancy control mechanism of some sort to deal with those changes." [6]

While [22] gives a value of $1063 \mathrm{~kg} \mathrm{~m}^{-3}$ for the density, [25] assumes a density ratio of plankton to seawater of 1.045 which means they're assuming a plankton density of about $1073 \mathrm{~kg} \mathrm{~m}^{-3}$. There are other similar estimates used in the literature (see [6], [50], [26]). All of these values are much higher than our value of 1027.23, but maintain the chosen sinking rate. A dynamic buoyancy could be introduced in a subsequent model, but for now we will assume a constant effective density rather than a measured one.

This gives us an $R$ value of

$$
R=\frac{m_{f}}{m_{p}+\frac{1}{2} m_{f}}=\frac{\rho_{f}}{\rho_{p}+\frac{1}{2} \rho_{f}}=\frac{1027}{1027.23+\frac{1}{2} 1027}=0.6665 \ldots<\frac{2}{3}
$$

making Euphausia pacifica a very slight $\left(R \approx \frac{2}{3}\right)$ aerosol. From now on when we refer to the "Stokes' inertia model" we mean the Stokes' inertia model with this $R$ value. For short times our plankton can be considered neutrally buoyant to a good approximation because the effects of both gravity and vorticity preference will be very small. On longer time scales simulations show that these small effects matter, as we will see in section 3. From our work in previous sections we know that the combined effect of our regular, low $Q$ flows, and fairly low St particles will be that the plankton quickly follow the sum of the flow and their swimming in between turning events while being continually pulled downward by the force of gravity.

\subsubsection{Biased Swimming}

As one final variant, we return to our swimming model and make a slight modification. Euphausia pacifica are advected away from $z_{L}$ by currents and so must swim to maintain this preferred light level. We model this return to preferred light level swimming as a modified version of the discrete run and tumble model. Instead of picking the angle of swimming as $\theta \in(0,2 \pi)$, we take $\theta \in(0, \pi)$ if the plankter is below the preferred light level, and $\theta \in(\pi, 2 \pi)$ if the plankter is above the preferred light level. Since the $z$ component of 
the swimming is given by $\sin (\theta)$, this means the plankter swims up if is below, and down if it is above, the preferred light level. The net effect is a discrete run and tumble model where the new direction is chosen so as to approach the preferred light level, albeit at a possibly oblique angle. As we shall see, this biased swimming has a profound effect on the dynamics.

\subsection{Completing the Models}

Let's review what we have determined so far. Recall that we had identified three key mechanisms that must be present in our plankton models

\section{Accurate Physics}

\section{Swimming}

\section{A Shear Response}

This analysis completes the basic description of both one and two on this list. We now consider the third item on the list before discussing some theoretical concerns.

\subsubsection{Plankton Response to Shear}

Several shear responses are considered in [25] (see their table 1), including an escape response, swimming down, and freezing and sinking. The authors chose the swimming down response as the most explanatory. However if the shear encountered is strong enough to interfere with navigation, or if currents are stronger than propulsion then this response is not possible. The response of stopping swimming (i.e. "freezing") in high shear is always an option, and this is the response we chose. For the fluid particle and neutrally buoyant Stokes' inertia models the freeze in shear response leads to pure advection of the particle. For the Stokes' inertia model, the freezing leads to a sinking at $0.5 \mathrm{~cm} / \mathrm{s}$, a response which Ianson et al. [25] call "freeze and sink" ( Figure 2.4 b)).

This alternate choice of shear response does not necessarily conflict with the findings of [25]. In their model only the bottom boundary would induce the shear response, but in 
our model there is potential for more of the domain to trigger the shear response, which means more time is spent in the shear triggered state of sinking. Put simply, sinking at a slow rate for a longer time could lead to similar population distributions to those resulting from swimming at a faster rate for a shorter time.

The dynamics of the models including this shear response are a hybrid of their deterministic and swimming versions. Where the shear is high, the swimming stops, and the time evolution of the system proceeds according to the models without swimming. Where the shear is low, the evolution proceeds according to the models which include swimming. This switching back and forth is visualized in the spacetime plot Figure 2.5. The dynamics of these systems were individually discussed above, and we will examine the results of including this response in section 3.

Since our plankton must respond to shear wherever they find it, how should shear be modelled, and what value of shear is "high enough" that the plankton stop swimming? Clearly in order to run a simulation that includes a shear response, we need a way to mathematically describe shear. The shear is calculated from the rate of strain tensor, and since it is a physical quantity, we need something to capture shear which is invariant under physical coordinate transformations. The rate of strain tensor has three invariants in three dimensions [20]. A quick calculation for a basic shear flow shows that the first and third invariants are zero, while the second invariant is not. For this reason we characterize shear using this invariant, as is done for non-Newtonian fluids (for example, see [44]). In two dimensions, this invariant is

$$
\begin{aligned}
I_{2}(x, z, t) & =\frac{\partial u(x, z, t)}{\partial x} \frac{\partial w(x, z, t)}{\partial z}-\frac{1}{4}\left(\frac{\partial u(x, z, t)}{\partial z}+\frac{\partial w(x, z, t)}{\partial x}\right)^{2} \\
& =\frac{\partial u}{\partial x} \frac{\partial w}{\partial z}-\frac{1}{4}\left(\frac{\partial u}{\partial z}+\frac{\partial w}{\partial x}\right)^{2}
\end{aligned}
$$

Written this way the first term captures the normal strain rates and the second term captures the shear strain rates. Both are important because it is reasonable to assume that an organism which would avoid shear would also avoid being crushed or torn apart. This scalar field quantifies the shear, and we can designate a critical value $c$, so that when $\left|I_{2}\right|>c$ the plankton consider the fluid to be high shear. The units of this scalar field are $1 / s^{2}$.

There does not appear to be any agreement on the value of the critical shear for zooplankton in the literature. As mentioned there are some qualitative theories on why 


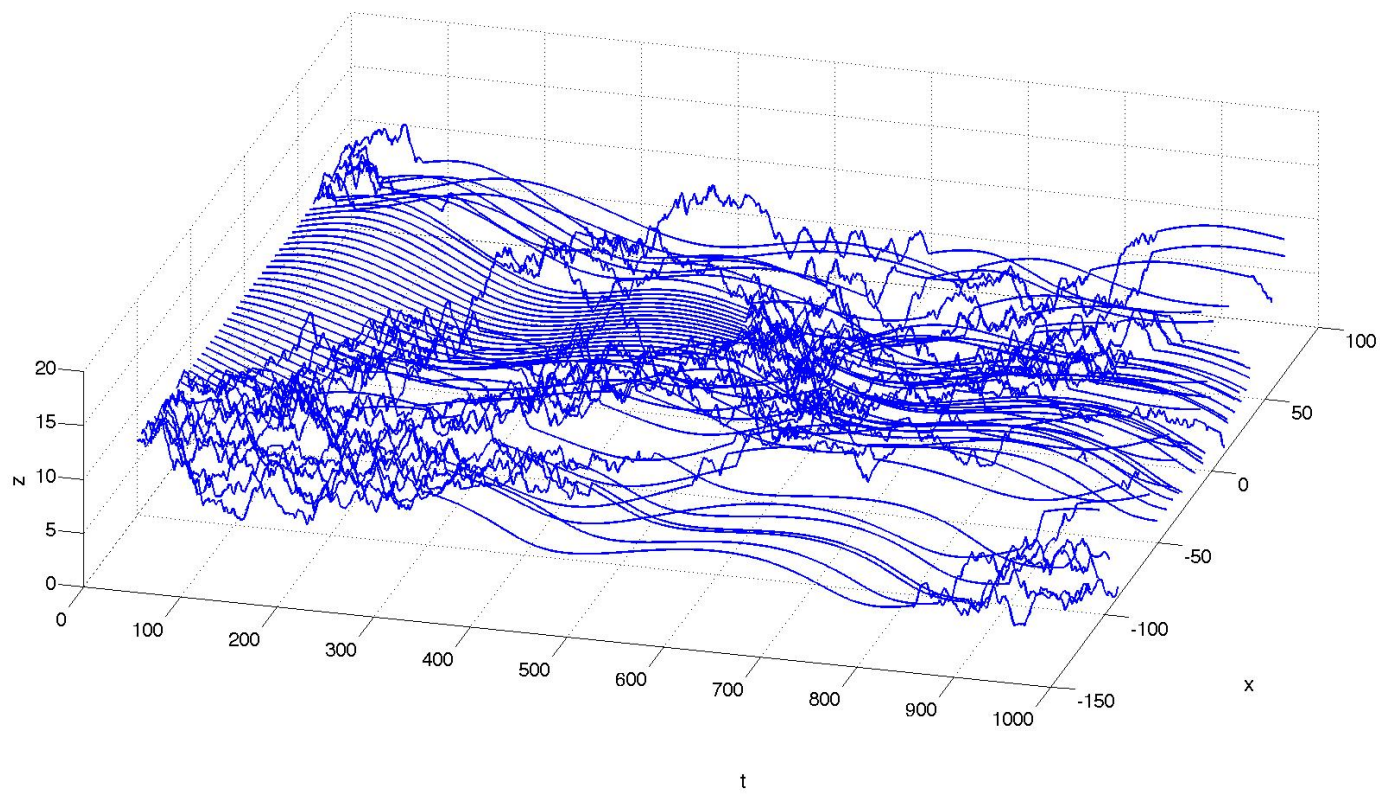

Figure 2.5: A spacetime plot of the fluid particle model with the freeze in shear response. The smooth curves from the linedrop at $t=0$ correspond to plankton which are frozen in shear, and so are deterministically advected by the flow, whereas the jagged curves correspond to the plankton in low shear which swim as they are advected. This effect can be seen to vary over time with the trajectory of any given plankter, as they switch back and forth between swimming and freezing in shear. 
plankton might not prefer high shear areas, but there are no quantitative results. Shear is

quantified using $\frac{\partial u}{\partial z}$ in [15] for the case of the phytoplankter $C$. nivalis and a critical value of 0.2 is chosen. In this case $\frac{\partial u}{\partial z}$ is objective because they have a shear flow, but in general this is not an objective measure. Additional barriers to comparison are that the shear's function in those simulations is to apply torque to the single celled phytoplankter rather than shear across the length of their bodies, and that there is no reason to think that two different sizes should have the same critical value. This if further exacerbated by the fact that in cases such as Knight Inlet, phytoplankton may live below the Kolmogorov scale while zooplankton may live above it. We came to the conclusion that it is best to test a range of critical values for shear affecting zooplankton to give us a small exploration of parameter space.

The Stokes' inertia model is the only model to include the effect of gravity, leading to an evolution of the population downward at the settling velocity $0.005 \mathrm{~m} / \mathrm{s}$ in still water when they are not swimming. However the plankton in this model only stop swimming in high shear and the water is not actually still in those regions. Instead, non-swimming plankton are accelerated to agree with a combination of the flow and the effect of gravity. If the vertical fluid speed is less than or equal to the settling velocity gravity dominates the evolution because the vertical advection is also downward some of the time, so that on average the net force is downward. Advection upward must compete against gravity, but must also be sustained to overcome the inertia of the plankton. The vertical component of the flows tested in section 3 oscillates, so those flows with mean vertical fluid speeds on the order of the settling velocity are still dominated by gravity because fully half of the time the flow is downward. For this reason we will call the regime where mean vertical fluid speeds are on the order of the settling velocity, or slower, "the settling regime." Note that plankton will only settle in the Stokes' inertia model, and then only in high shear regions.

\subsubsection{Theoretical Concerns}

Technically, in order to use the Maxey-Riley equations with confidence we must first check that our model satisfies the basic assumptions that the equations are based on. The Maxey-Riley equaitons assume the particle diameter is much less than the Kolmogorov length scale. As mentioned, we're off by at least an order of magnitude here. It is also assumed that the shear Reynolds number $R e_{\Gamma}=a^{2} \Gamma / \nu<<1$ where $\Gamma$ is a typical velocity gradient for the flow. We tested a range of flows which had the same geometric structure 
but different speeds, and found that $R e_{\Gamma}<0.5$ was satisfied for all flows, which we consider acceptable.

Another assumption is that the particle Reynolds number, $R e_{p}=a|\mathbf{v}-\mathbf{u}| / \nu$ will remain small. We initialize the model with $\mathbf{v}=\mathbf{u}$ to ensure that $R e_{p}$ starts small. Depending on fluid speeds, plankton swimming speeds may not be small in comparison, so that $R e_{p}$ may not remain small. We find that the average $R e_{p}$ over the range of flows tested is under 350 for swimming plankton, although faster flows included particle Reynolds numbers as high as 6250. This is far too high. We can note that we are not employing the full Maxey-Riley equations, and so it is probably better to use the validity of Stokes' drag as a guide for the validity of these equations, since it is the only force besides gravity considered. However Stokes drag assumes low Reynolds numbers which is an assumption we do not satisfy. There are corrections available making a Stokes drag type equation valid for various ranges of particle numbers [37]. Alternatively, in a future modification of the model we could move to a more general drag force such as the drag equation $F_{d}=$ $\frac{\pi}{2} \rho_{f}(\mathbf{v}-\mathbf{u})^{2} c_{d} a^{2}$ (see [31]). This would require a value for the drag coefficient $c_{d}$, which depends on the Reynolds number. The values of $c_{d}$ are not based on a theoretical derivation, but on phenomenological observation. Values for $c_{d}$ at high Reynolds number have been calculated in [1]. However, the Stokes' drag has the advantage that it does not have such a coefficient, but is describable in terms of known quantities based on the flow and particle properties. Additionally, all of these alternatives for the drag force employed in this paper are corrections for spherical particles, and Euphausia pacifica, as well as many other zooplankton, are not close to spherical. These organisms are also changing shape as they constantly reorient and propel themselves. There are many possible avenues for improvement on drag value accuracy which could be examined in future work. 


\section{Chapter 3}

\section{Results}

\subsection{Setup}

\subsubsection{Parameter Space}

We chose to look at four critical values for the shear, and a range of flows for each. To capture the interaction of swimming with the high shear response, for each critical value we considered three flows: one for which $25 \%$ the domain was considered high shear according to $I_{2}$, one for which it was considered $50 \%$ high shear, and one for which it was considered $75 \%$ high shear. To minimize variation between cases, we elected to change the speed of the flow only. This was accomplished by making the amplitudes in (1.9) dependent on a third parameter $s$ in the following way:

$$
\begin{aligned}
& A_{1}=\alpha s \\
& A_{2}=s \sqrt{1-\alpha^{2}}
\end{aligned}
$$

where $\alpha=0.9$ was a fixed splitting factor. Varying $s$ generates a one parameter family of solutions which vary the speed of the fluid, but not the geometry of the streamlines. This allows us to take values of $s$ corresponding to specific quantiles of area considered high shear by the plankton for different critical values. Plots of the percentage of the domain considered high shear by the plankton, as a function of $s$, are shown in Figure 3.1. 


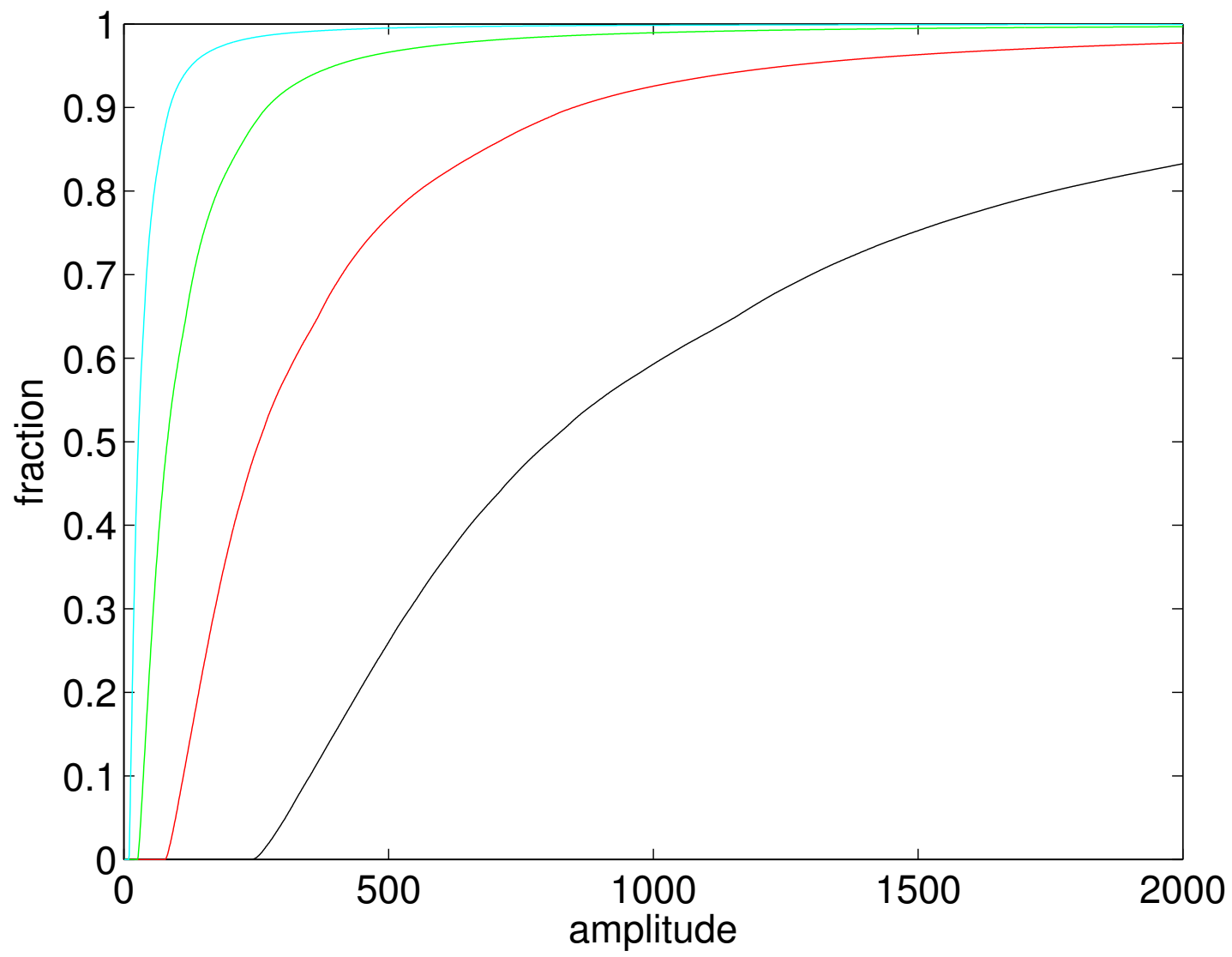

Figure 3.1: Fraction of the domain considered high shear as a function of $s$ in centimeters. Lighter curves correspond to smaller critical shear values. All curves asymptote to filling the domain with high shear, but curves for larger critical values do so more slowly.

Tables 3.1 and 3.2 give some basic metrics of these flows. By choosing values to get the quantiles we want, we have also chosen twelve flows of increasing speed. By panel b) of Table 3.1 we see that the settling regime for this exploration of parameter space is the slowest five flows. Since the swimming speed of the plankton is fixed at $v_{\text {swim }}=0.1 \mathrm{~m} / \mathrm{s}$, from Table 3.2 we see that the flows range in speed from an order of magnitude slower than that of the plankton, to an order faster. Shear quantile stills are shown in Figure 3.2. 


\begin{tabular}{|l|l|l|l|}
\hline critical value $\left(\frac{1}{s^{2}}\right)$ & $25 \%$ & $50 \%$ & $75 \%$ \\
\hline $5 \times 10^{-6}$ & 0.16 & 0.26 & 0.48 \\
\hline $5 \times 10^{-5}$ & 0.5 & 0.81 & 1.49 \\
\hline $5 \times 10^{-4}$ & 1.56 & 2.54 & 4.71 \\
\hline $5 \times 10^{-3}$ & 4.91 & 8.03 & 14.87 \\
\hline
\end{tabular}

(a)

\begin{tabular}{|l|l|l|l|}
\hline critical value $\left(\frac{1}{s^{2}}\right)$ & $25 \%$ & $50 \%$ & $75 \%$ \\
\hline $5 \times 10^{-6}$ & 0.0019 & 0.0030 & 0.0056 \\
\hline $5 \times 10^{-5}$ & 0.0058 & 0.0094 & 0.0173 \\
\hline $5 \times 10^{-4}$ & 0.0181 & 0.0295 & 0.0547 \\
\hline $5 \times 10^{-3}$ & 0.0570 & 0.0932 & 0.1725 \\
\hline
\end{tabular}

(b)

Table 3.1: Values of $s$ corresponding to high shear area quantiles for the four critical values tested are shown in panel a). Mean vertical fluid speeds over the domain at $t=0$ are shown in panel b), by the definition in the text, these values show that for this parameter space exploration the settling regime consists of the slowest five flows.

\begin{tabular}{|l|l|l|l||l|l|l|l|}
\hline critical value $\left(\frac{1}{s^{2}}\right)$ & $25 \%$ & $50 \%$ & $75 \%$ & critical value $\left(\frac{1}{s^{2}}\right)$ & $25 \%$ & $50 \%$ & $75 \%$ \\
\hline $5 \times 10^{-6}$ & 0.0445 & 0.0724 & 0.1336 & $5 \times 10^{-6}$ & 0.0000 & 0.0001 & 0.0002 \\
\hline $5 \times 10^{-5}$ & 0.1392 & 0.2254 & 0.4147 & $5 \times 10^{-5}$ & 0.0002 & 0.0006 & 0.0019 \\
\hline $5 \times 10^{-4}$ & 0.4342 & 0.7069 & 1.3108 & $5 \times 10^{-4}$ & 0.0021 & 0.0055 & 0.0191 \\
\hline $5 \times 10^{-3}$ & 1.3665 & 2.2348 & 4.1385 & $5 \times 10^{-3}$ & 0.0207 & 0.0554 & 0.1901 \\
\hline
\end{tabular}

(a)

Table 3.2: Maximum speeds at $t=0$ in $\mathrm{m} / \mathrm{s}$ are shown in panel a). Max values of $\left|I_{2}\right|$ over the domain at $t=0$ are shown in panel $\mathrm{b}$ ). Note the max shear is smaller than our accuracy in the first row of $b$ ).

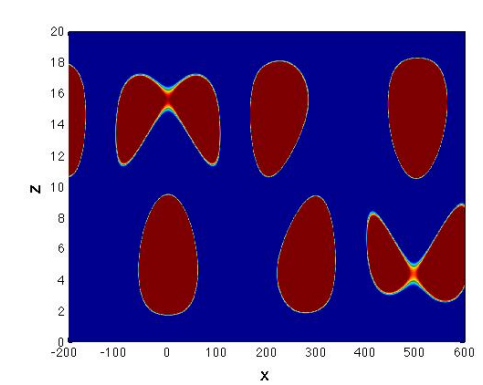

Figure 3.2: Red represents high shear regions. From left to right these are $25 \%, 50 \%$, and $75 \%$ high shear quantiles.

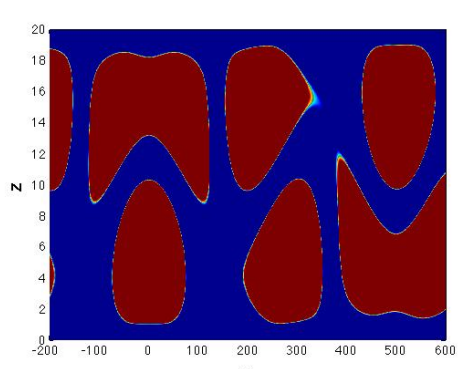

$x$

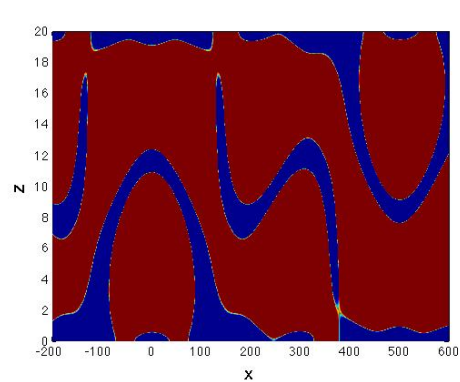
$25 \%, 50 \%$, and 
In the analysis that follows, when we refer to "the range of flows" we mean these 12 .

As shown in equation 1.9, a superposition of two solutions was considered: a mode one $\left(m_{1}=1\right)$ solution of amplitude $A_{1}$ and a mode two $\left(m_{2}=2\right)$ perturbation of amplitude $A_{2}$, where $A_{1}>A_{2}$. Each of the two solutions in the superposition had fixed frequencies $\sigma_{i}$ and fixed wavenumbers $k_{i}$ and $m_{i}$ across all simulations, so that the phase speeds of each solution in the superposition were also fixed across all simulations. The phase speed of the primary solution in the superposition is

$$
V=\frac{\sigma_{1}}{k_{1}}=0.1249 \mathrm{~m} / \mathrm{s}
$$

This is the number we will use in our analysis because the phase speed $\frac{\sigma_{2}}{k_{2}}=0.0636$ $\mathrm{m} / \mathrm{s}$ of the perturbation determines a small deformation of the primary solution which travels slower than the primary solution. This means that $V$ is the speed of interest when considering transport and trapping because it gives a good estimate of the peak waveinduced speed. An alternative would be to use the weighted average of the solutions to give

$$
\frac{\alpha V+\sqrt{1-\alpha^{2}} 0.0636}{\alpha+\sqrt{1-\alpha^{2}}} \approx 0.1048 \mathrm{~m} / \mathrm{s}<V
$$

as we might expect since the perturbation is slower than the primary solution. For our small exploration of parameter space either value would have worked, so we will stick with $V$. The purpose of this value is to give a rough baseline value for the Lagrangian frame speed, and it serves this purpose well.

Having each solution propagate at a different speed provides a shear field which evolves in time rather than simply propagating. It also breaks the symmetry to better approximate the background flow as a version of the flow depicted in Figures 10 to 17 of [33]. 

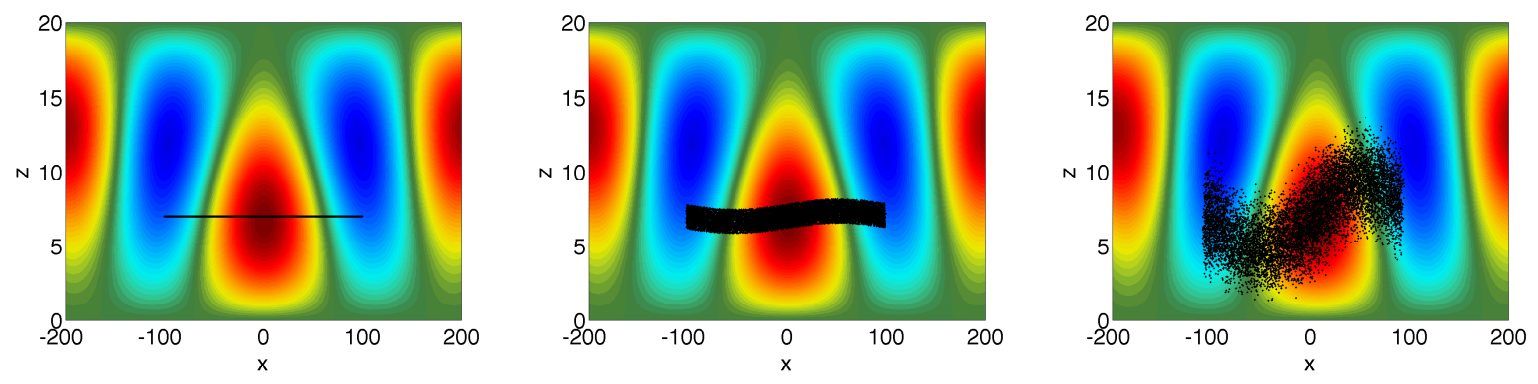

Figure 3.3: The initial position of the plankton is shown in the first panel, the second panel is at $t=8 \mathrm{~s}$, the third is at $t=56 \mathrm{~s}$. This is the base fluid particle model with swimming, outlined in section 2.2.2. This flow is the first quantile of the $5 \times 10^{-4}$ critical value, so a medium speed flow from the regime. The red gyres are counterclockwise and the blue gyres are clockwise which clearly advects the plankton cloud as they swim.

Every simulation begins as in Figure 3.3: the plankton are dropped at their preferred light level and begin to swim while being advected by the flow according to the model which has been selected from section 2. The simulation then runs for 4000 seconds.

\subsubsection{Model Selection}

Many versions of the models described in section 2 were considered, some of which yielded no useful results. For any attribute of a population, including swimming speed or shear response, variance of that attribute within the population is expected on biological grounds. Variants of the fluid particle model which included variation by introducing substantial variance in both critical shear level and swim speed were considered. No meaningful differences were found in large ensembles. There were small quantifiable differences, such as slight variations in scalar measures, but no overall trends to speak of. Population variation can easily be added again at any stage, but for now populations without it are considered so that the effects of the mechanisms at work can be identified as easily as possible. For this reason we focus on the 12 models from Table 3.3. From now on when we refer to "the three models" we mean Stokes' inertia with the $R$ value calculated in 2.2.5 (making the plankton a slight aerosol), neutral buoyancy Stokes' inertia, and the fluid particle model. Finally, when we consider any of the three models without either a shear response or biased swimming we will call that variant "the base model." 


\begin{tabular}{|l|l|l|}
\hline Model Options & Freeze & Bias \\
\hline Stokes' inertia & yes / no & yes / no \\
\hline neutral buoyancy Stokes' inertia & yes / no & yes / no \\
\hline Fluid Particle & yes / no & yes / no \\
\hline
\end{tabular}

Table 3.3: We consider models corresponding to all possible ways of filling out this chart, so each model has the four choices of no freeze or biased swimming, both, or only one of freeze or biased swimming, for a total of 12 models.

\subsubsection{Tools}

One classical metric when dealing with a randomly walking population is to consider the (total) variance (i.e. the trace of the covariance matrix) as a function of time. In the classical analysis if there is no drift and the initial conditions of the Fokker-Planck equation are taken to be a delta function of position, so that it is assumed the initial position of the plankton is a single point. The motion of the population then follows a diffusion process, featuring a linear increase of variance over time. It is unsurprising that one parameter should capture the growth of a diffusion process because of the isotropy of the process. The introduction of a background drift can have profound consequences on the geometry of a diffusing population, and so variance becomes an inadequate tool for analyzing these more diverse geometries. What we found was that any anomaly in the variance plots required us to return to the animations for an explanation anyway. For our situation variance plots were an analytic tool with no predictive or descriptive power. See 4.5 in the appendix for a longer discussion.

Variance was not the only measure we tried. We also considered interparticle distances, so that the ensembles were tracked in pairs. Interpreting the output of the simple measures we tried always required reference back to the simulations, so that those measures were of no use in isolation. It is certainly possible that a useful measure could be constructed, but we were unable to do so within the confines of the Master's duration. Perhaps in the Doctorate.

That said, the most powerful tool we have is also the simplest: animations of the results of our numerical experiments. These animations are the analogues of film of physical 
experiments, and allow us to carry out all of the same analyses. Science depends on observations, and direct observations depend on the use of our eyes, so that science has a long history of dependence on our visual analytical abilities. Fortunately, the human eye is able to observe patterns subtle enough that is is difficult to express them in any language, including that of mathematics. This is what makes the animations so useful.

\subsection{Analysis of the Mechanisms}

Our models and their variants employ the mechanisms of a freezing in shear response and of swimming in both unbiased and biased versions. Although our exploration of parameter space is small, when combined with the different models and their variants we have many cases to consider. To make progress in our analysis we first observe a similarity between two of our three models, and then build up our intuition of, and nomenclature for, the differences and similarities of all the models and their variants, and what we observe in the plankton populations we simulate by them.

\subsubsection{Comparing the Fluid Particle and Neutrally Buoyant Stokes' Inertia Models}

As mentioned in section 2.1.5, the fluid particle model and the neutrally buoyant Stokes' inertia models $\left(R=\frac{2}{3}\right)$ agree well over all the variants, and over the range of flows tested. The dynamics of the models cannot actually be the same, as the following thought experiments show: take both models and include a shear response and suppose a plankter from each population is being accelerated along an integral curve contained inside a high shear region. Assume this integral curve turns sharply near the boundary of that region to stay inside it. In this case the inertia of the Stokes' inertia model plankter can carry it out of the high shear region while the inertialess fluid particle plankter remains inside. This is the situation in Figure 3.4 a). Similarly, motion along an integral curve inside a high shear region travelling parallel to the boundary and then sharply crossing it could carry the inertialess plankter with it, but leave the Stokes' inertia plankter inside. This is the case in Figure 3.4 b). 

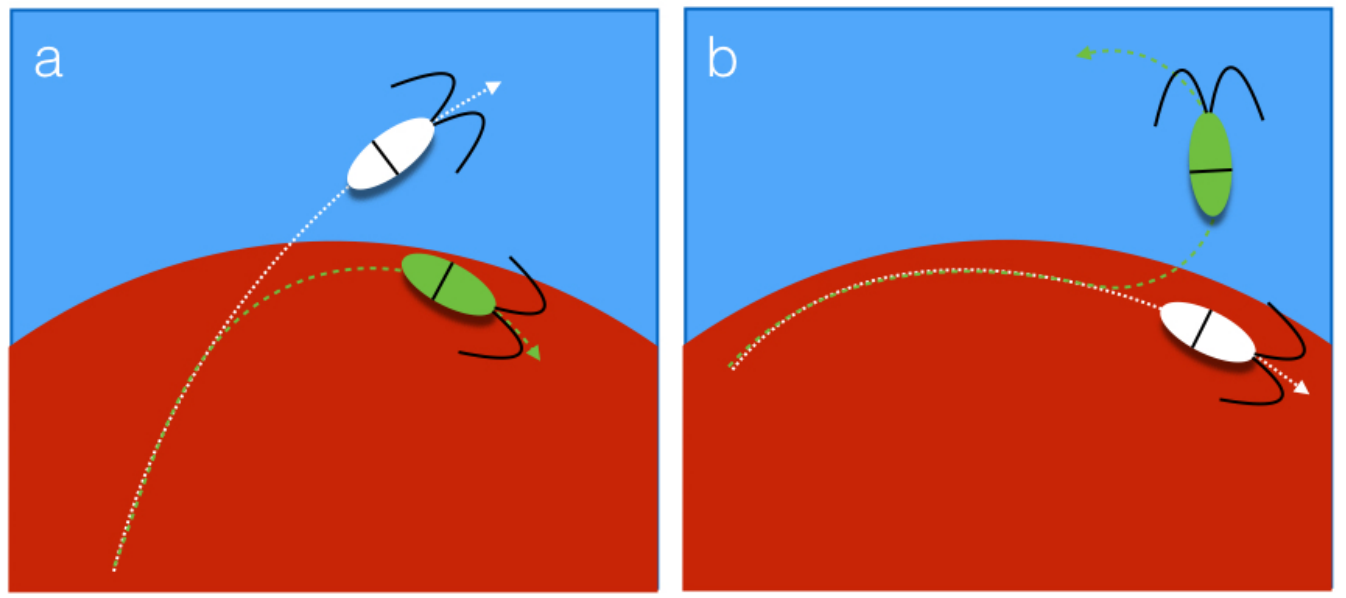

Figure 3.4: Red is high shear and blue is low shear. Panel a) shows inertia carrying a white plankter modelled under the neutrally buoyant Stokes' inertia model out of a high shear region while a plankter modelled under the fluid particle (in green) follows the integral curve and stays inside. Panel b) shows inertia keeping a plankter inside a high shear region.

For the two fastest flows the variant with both a shear response and biased swimming showed a separation of these models significant enough to be worthy of mention, but over the range of flows tested, the neutrally buoyant Stokes' inertia and fluid particle models agreed extremely well in the majority of cases. 


\subsubsection{Trapping: Base Models}

Consider the three base models. Our flows translate at the same speed $V=0.1249 \mathrm{~m} / \mathrm{s}$ $>v_{\text {swim }}=0.1 \mathrm{~m} / \mathrm{s}$. Lamb [33] points out that, in the fluid particle model, if the horizontal fluid speed $u(x, z, t)+u_{\text {swim }} \geq V$ for a constant $u_{\text {swim }}$, plankton will be trapped in the wave and advected along with it. In our situation the swimming speed is not constant but the maximum possible swim speed in the horizontal direction is $v_{\text {swim }}=0.1 \mathrm{~m} / \mathrm{s}$. Therefore if $u(x, z, t)-v_{\text {swim }} \geq V$ the population of plankton is trapped and transported by the wave. If $u(x, z, t)-v_{\text {swim }}<V$ plankton are not trapped. Trapping occurred in all three base models for the eight fastest flows. This was predicted accurately by the inequality condition used with the values of Table 3.4 a).

\begin{tabular}{|l|l|l|l||l|l|l|l|}
\hline critical value $\left(\frac{1}{s^{2}}\right)$ & $25 \%$ & $50 \%$ & $75 \%$ & critical value $\left(\frac{1}{s^{2}}\right)$ & $25 \%$ & $50 \%$ & $75 \%$ \\
\hline $5 \times 10^{-6}$ & 0.0445 & 0.0724 & 0.1336 & $5 \times 10^{-6}$ & 0.0048 & 0.0078 & 0.0144 \\
\hline $5 \times 10^{-5}$ & 0.1392 & 0.2254 & 0.4147 & $5 \times 10^{-5}$ & 0.0150 & 0.0243 & 0.0446 \\
\hline $5 \times 10^{-4}$ & 0.4342 & 0.7069 & 1.3108 & $5 \times 10^{-4}$ & 0.0467 & 0.0760 & 0.1410 \\
\hline $5 \times 10^{-3}$ & 1.3665 & 2.2348 & 4.1385 & $5 \times 10^{-3}$ & 0.1470 & 0.2404 & 0.4452 \\
\hline
\end{tabular}

(a)

Table 3.4: Maximum values of the components of the velocity field at $t=0$. Panel (a) shows the horizontal component and (b) shows the vertical. By smoothness and periodicity we take these values to be representative of the values over the entire time domain.

Where trapping exists, it often exhibits a geometric character. There are points where $u(x, z, t)=0$ and by spatial periodicity of the flow these points are in coherent bands where $u(x, z, t) \approx 0$. As plankton approach a band, $u$ diminishes, and we have $u(x, z, t)+v_{\text {swim }} \leq$ $V$, because $v_{\text {swim }}<V$ and $u$ is small. In this case the region where $u \approx 0$ becomes a kind of stable region: plankton swim slower than the wave, so if they approach this region from behind they cannot pass it on their own, and if they fall behind this region $u$ can increase to where $u>V$ and they are accelerated towards it. Similarly if they approach this region from the front they cannot fall too far behind it. This stability creates a sharp boundary which is most clear in the Stokes' inertia model because gravity causes that population to congregate near the bottom of the channel. These band formations are a kind of trapping, and so were only observed in the fastest eight flows, as seen in Table 3.4. Note that this table also justifies why the horizontal flow components are considered in the trapping analysis: the vertical components of the flow are an order of magnitude weaker 
than the horizontal components. To summarize, we have found that the eight fastest flows exhibit trapping in band formations for all three base models.

\subsubsection{Trapping: Biased Swimming}

Now consider all three models with biased swimming towards the preferred light level of $z_{L}=7 \mathrm{~m}$ but no shear response. In this case the most relevant speeds for trapping are not the maximum horizontal speeds throughout the domain, but those at the preferred light level. This gives us Table 3.5.

\begin{tabular}{|l|l|l|l||l|l|l|l|}
\hline critical value $\left(\frac{1}{s^{2}}\right)$ & $25 \%$ & $50 \%$ & $75 \%$ & critical value $\left(\frac{1}{s^{2}}\right)$ & $25 \%$ & $50 \%$ & $75 \%$ \\
\hline $5 \times 10^{-6}$ & 0.0204 & 0.0332 & 0.0613 & $5 \times 10^{-6}$ & 0.0044 & 0.0072 & 0.0133 \\
\hline $5 \times 10^{-5}$ & 0.0639 & 0.1035 & 0.1904 & $5 \times 10^{-5}$ & 0.0139 & 0.0225 & 0.0414 \\
\hline $5 \times 10^{-4}$ & 0.1993 & 0.3246 & 0.6019 & $5 \times 10^{-4}$ & 0.0433 & 0.0705 & 0.1308 \\
\hline $5 \times 10^{-3}$ & 0.6247 & 1.0261 & 1.9001 & $5 \times 10^{-3}$ & 0.1364 & 0.2230 & 0.4130 \\
\hline
\end{tabular}

(a)

Table 3.5: Maximum values of the components of the velocity field at $t=0$ and $z=7 \mathrm{~m}$. Panel (a) shows the horizontal component and (b) shows the vertical. By smoothness we take these values to be representative of the values near $z=7$ as well. By periodicity we take these values to be representative of the values over the entire time domain.

The biased swimming behaviour causes the line $z=7 \mathrm{~m}$ to be a stable region for the plankton because they always swim back to it. The only mechanism that can perturb the plankton away from this line is a strong vertical current. Note that since the swimming is not directly up or down, the mean swimming speed up or down when away from the preferred light level is

$$
\bar{v}=\left\langle v_{\text {swim }} \sin (\theta)\right\rangle_{\theta}=\frac{v_{\text {swim }}}{\pi} \int_{0}^{\pi} \sin (\theta) d \theta \approx 0.06366
$$

Table 3.5 shows that the slowest seven flows satisfy $w(x, 7, t)<\bar{v}$, which results in a layer of plankton at $z_{L}=7$. In this case the same trapping analysis as in section 3.2 .2 can be 
applied: trapping occurs when $u\left(x, z_{L}, t\right)-v_{\text {swim }} \geq V$, which we see from Table 3.5 is true for the fastest five flows. Once again this inequality condition predicted the presence of trapping in simulations. This means that trapping does not occur for the flows satisfying $w\left(x, z_{L}, t\right)<\bar{v}$. Trapping does occur for the flows where $w\left(x, z_{L}, t\right) \geq \bar{v}$, but since the vertical currents are also strong we get a more interesting structure to the population than a simple layer of plankton at $z_{L}$. We also get trapping in gyres for the fastest four flows. This will be discussed further in section 3.2.7.

\subsubsection{Trapping: Shear Response}

Now consider all three models with the freeze in shear response, but no biased swimming. Since plankton do not swim when they're in high shear, the trapping requirements are different inside and outside of the high shear regions. Outside we still have the $u(x, z, t)-$

$v_{\text {swim }} \geq V$ requirement, but inside $u(x, z, t) \geq V$ is sufficient. This latter requirement is satisfied for the fastest ten flows as we can see in Table 3.4. This table also outlines how much of the domain satisfies each inequality. So for example the $25 \%$ quantile flow for the $5 \times 10^{-5}$ critical value meets the trapping criteria for the $25 \%$ of the domain that is high shear, but not for the $75 \%$ of the domain which is low shear. This example also shows another subtlety, as the fastest horizontal currents occur along the bottom boundary, where the free-slip conditions cause low shear. In that flow we do not observe trapping because the high shear region and maximal horizontal fluid speed region do not coincide, but we do get what is essentially trapping as depicted in the left panel of Figure 3.5 for all three models. 

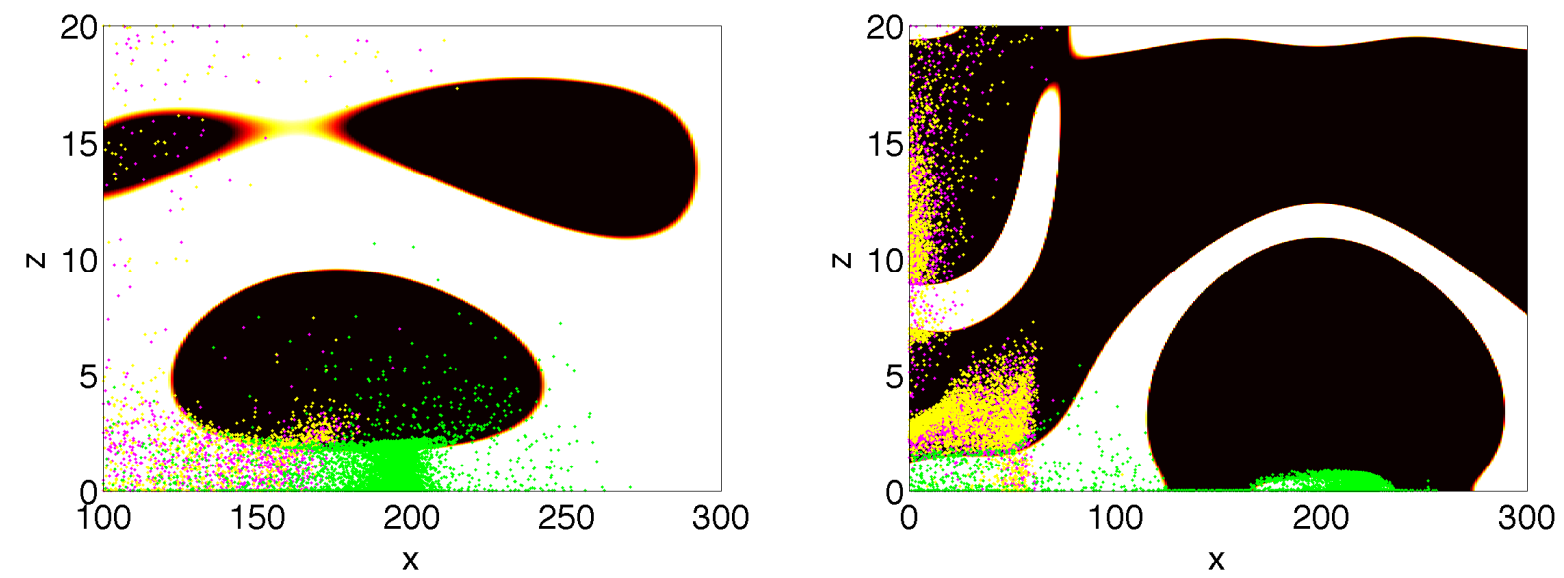

Figure 3.5: The fluid particle model is shown in magenta, the neutral buoyancy Stokes' inertia in yellow, and the Stokes inertia in green. In the left panel $(t=3000 \mathrm{~s})$ we see all three populations caught below a region of high shear, shown in black. As they swim up they are advected by the high shear zone and the net effect is that they are essentially trapped despite being predomininantly outside the high shear region. The right panel $(t=3200 \mathrm{~s})$ shows the Stokes inertia model trapped between the bottom boundary and a high shear region enclosing it.

As another example, for the $75 \%$ quantile $5 \times 10^{-6}$ flow we get trapping for the Stokes' inertia model only, where gravity has caused the separation that led to a population being trapped between high shear and the bottom boundary. This trapping could, in principle, also occur in the other two models, it is just that in the Stokes' inertia model gravity led to the population being in the appropriate location to begin with. We therefore conclude that in our flows $u(x, z, t) \geq V$ is a good indicator of the presence of trapping, and we see that the ten fastest flows satisfy this criteria by looking at Table 3.4. These examples show that the varying trapping criteria over the domain makes predicting the presence of trapping effects difficult, but that trapping occurred in the $u(x, z, t)-v_{\text {swim }} \geq V$ sense in the eight fastest flows, and by shear trapping in the cases mentioned, so that the ten fastest flows exhibit trapping. We also found that the force of gravity caused significant separation of the Stokes' inertia model from the fluid particle and neutrally buoyant Stokes' inertia models in the variant with a shear response and unbiased swimming. We will return to this in section 3.2.8. 


\subsubsection{Trapping: Biased Swimming and Shear Response}

Finally, we consider all three models with both a shear response and biased swimming. Once again it is Table 3.5 which is most relevant. Following the same analysis as for the shear response with unbiased swimming variants we expect trapping in the seven fastest flows because those are the flows for which $u\left(x, z_{L}, t\right) \geq V$ holds. Experiments show that this is the case.

\subsubsection{Trapping in Gyres}

A stronger type of trapping occurs if the fluid speed is much faster than the wave speed. In this case the integral curves of the gyres are nearly closed (in a frame moving with the wave), and the plankton get caught in gyres. This occurred in all three models and in all variants, provided the flow was fast enough. Trapping in gyres requires faster fluid speeds than trapping in bands but how fast depends on the model variant: the variant with biased swimming but no shear response only showed this effect in the fastest four flows, as we will discuss in section 3.2.7. The variants with the shear response had this effect occur in the fastest seven flows, so in those flows outside the settling regime. The models with the shear response have less swimming overall, and so are trapped more easily. Trapping in gyres is more difficult to quantify, and so we base our analysis on the more direct trapping in bands analysis, since it can be characterized by tables like 3.4.

\subsubsection{Light Attractors}

As mentioned in section 3.2.3, for all three models with biased swimming and no shear response when $w\left(x, z_{L}, t\right) \geq \bar{v}$ as for the fastest five flows, we get very different dynamics than for the $w\left(x, z_{L}, t\right)<\bar{v}$ case. By Table 3.5, trapping occurs for all five of these flows, giving us three mechanisms: biased swimming and currents stronger than swimming in the vertical, and strong currents in the horizontal. The interplay of these mechanisms leads to coherent structures of plankton that we will refer to collectively as "light attractors", a name chosen by analogy with attractors of dynamical systems.

For the slowest of the five flows satisfying $w\left(x, z_{L}, t\right) \geq \bar{v}, w\left(x, z_{L}, t\right) \approx \bar{v}$ and the perturbation by the flow is small, leading to a trapping in a band as described in 3.2 .2 
1. Over the four fastest flows we tested, light attractors do not form in bands because bands form at regions where the vertical currents are strongest, and in particular are much stronger than the swimming of the plankton. However, at the centre of gyres the vertical currents are small enough that the swimming can overcome them. As the plankton are perturbed by the vertical currents and pass through the domain, they are constantly swimming toward their preferred light level $z_{L}$. If they reach $z_{L}$ near the center of a gyre, the vertical currents are too weak to perturb them away from $z_{L}$. Additionally, horizontal currents are at their weakest at the center of a gyre, but are still an order of magnitude stronger than the vertical currents. This means that near the center of a gyre is a region where the vertical currents are smaller than the swim speed, but the horizontal currents are faster than the swim speed. If $z_{L}$ is near the center of the gyre then we have a region where the swimming is essentially in the vertical and the advection is in the horizontal but in opposite directions above and below the gyre. The bias in the swimming causes them to alternate above and below $z_{L}$ at the center of the gyre, which means they alternate the direction of their advection as well. Finally, since currents increase in strength away from the center of the gyre, the further away from the center of the gyre they get the stronger the restoring force. This process induces a light attractor at the center of gyres whose centers are near $z_{L}$, and the gyres themselves are advected at the wave speed $V$. We will call these light attractors "stable light attractors." This does not mean they cannot be destroyed. The gyres are not static as they are advected, and as they change shape they may do so in such a way that their center is no longer close to $z_{L}$. Additionally, since the trapping of these attractors does not occur at the maximum horizontal velocities, it is possible for them to drift out of the center of the gyre as it travels, meaning the trapping is enforced elsewhere. Still, these light attractors are stable in the sense that if they are advected in a stable gyre they will persist.

\footnotetext{
${ }^{1}$ We will not give this structure a special name. It is essentially the layer of plankton observed in the slowest seven flows, but collected into a smaller region by the trapping mechanism.
} 

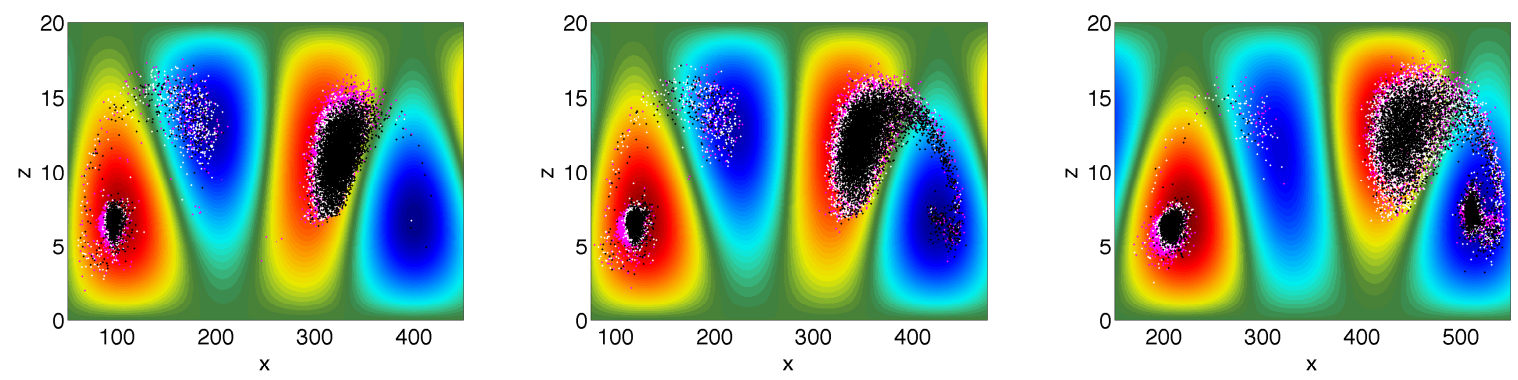

Figure 3.6: We see patches of all three populations, in black, white, and magenta, in the form of two unstable and stable light attractor pairs. Times from left to right are 2396, 2596, and $3356 \mathrm{~s}$. The unstable light attractors in the middle two gyres feed the stable light attractors in the outer two gyres, and in the end the left unstable attractor has almost been destroyed by this process. Note the horizontal axes: this process occurs in the Lagrangian frame.

For stable light attractors, the stabilization is a result of the alternating of the plankton above and below $z_{L}$. If $z_{L}$ is not at the center of the gyre but is still within it, the plankton's swimming towards $z_{L}$ leads to the plankton filling and often spilling out of the gyre. As the plankton swim towards $z_{L}$ they get closer to the edge of the gyre, and the edge of the gyre includes more integral curves which leave it. Simulations show that for gyres with centers above $z_{L}$, plankton form disks which leaked plankton into adjacent gyres, whose centers were closer to $z_{L}$, to form primary light attractors. Since these structures are the result of biased swimming, we still call them light attractors. However, due to their tendency to feed into stable light attractors we call these "unstable light attractors." Both of these are shown in Figure 3.6.

The existence and stability of light attractors depends very much on the flow geometry and nature of the preferred light level. Our flows are a one parameter set of linear solutions to the internal wave problem, and as such are a series of gyres of approximately the same size and shape. More complicated flows, especially those featuring turbulence, or different $Q$ or $S t$ values, could be entirely different. Although our flows represent a simple case, we know that many flows are smooth to a good approximation, and could feature common structures like gyres. Additionally, had we chosen a preferred light level which did not coincide with the center of some of our gyres, it is possible that we may have had no stable light attractors at all. Our terms are relatively loose, as we've seen that stable attractors can be destroyed, and unstable attractors are eventually destroyed, but these structures are difficult to describe simply and we feel these terms capture the intuition of the situation. 


\subsubsection{Shear Attractors}

Now consider all three models without biased swimming but with the freezing in shear response. This response induces another set of coherent structures of plankton which we will call "shear attractors." Across the range of flows tested, shear attractors resulted from the freezing in shear response by one of two mechanisms. The first can be described as follows: a small band of integral curves of the flow which pass out of a region of high shear and then back in again collect plankton by advecting them into the high shear region. The plankton are then carried through the high shear region until they are advected out of the high shear region close to their original entry point ${ }^{2}$. Their next few swimming decisions are crucial. Since the swimming decisions are unbiased, if they swim back into the high shear region they will be frozen and advected through it again, and if they swim away from it they may not swim far enough to avoid being pulled back in anyway. This creates coherent structures of rings of plankton advected by the flow as in the top left panel of Figure 3.7. Clearly the formation and shape of these structures is highly dependent on the flow geometry, but these were observed in the seven fastest flows in all three models and constitute a form of trapping as discussed in section 3.2.6.

\footnotetext{
${ }^{2}$ Close in Lagrangian coordinates. The entire flow may have been translated, but the structure has retained its identity.
} 

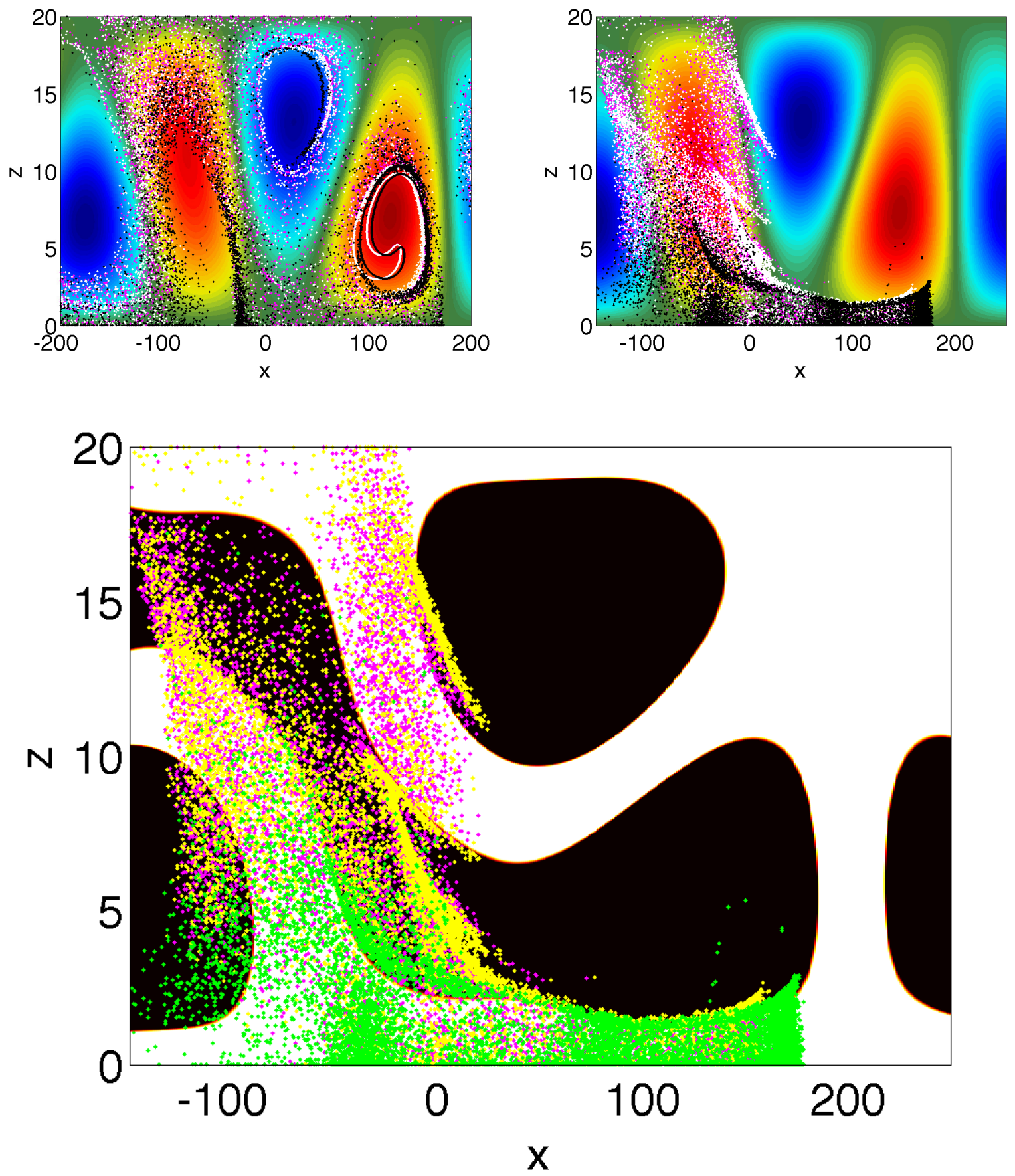

Figure 3.7: The top left panel $(t=1000 \mathrm{~s})$ shows patch formation by ring shaped shear attractors for all populations: fluid particle in magenta, neutrally buoyant Stokes' inertia in white, and Stokes' inertia in black. Note the trapping in a band as well around $x=-25$. The top right and bottom panels $\left(t=1200^{51}\right)$ show filament shear attractors. The third panel shows the the shear field plotted at the same time as the second panel, high shear in black and low shear in white. The color of neutrally buoyant Stokes' inertia has been changed from white to yellow and the color of Stokes' inertia has been changed from black to green. This panel clearly shows the separation of the Stokes' inertia model (green) from the other two (yellow and magenta), as well as trapping below the high shear region. 
The second mechanism for generating shear attractors is more subtle. The interaction of the collection of plankton along high shear region boundaries, advection, swimming in low shear regions, and, in the case of the Stokes' inertia model, gravity, combine to give a myriad of coherent structures of plankton which we will also call shear attractors. This is a large grouping to make, but they do have a common defining feature: filaments of plankton whose thickness depends on the shear quantile. Some examples of these filament structures are shown in the bottom panel of Figure 3.7, where the dependence on the amount of shear has clearly influenced the shape, particularly along the bottom boundary. This makes sense because plankton swim in low shear, diffusing to fill the domain over time. If the low shear covers only $25 \%$ of the domain it occurs in narrower structures than if it was $50 \%$ or $75 \%$. In the six slowest flows, the effect of gravity in the Stokes' inertia model causes significantly different population dynamics than for the models without inertia. In particular, the concentration of that population along the bottom boundary was blocked from above by high shear regions and advected back down, similar to the shear trapping mechanism described in section 3.2.4. See the top right and bottom panels of Figure 3.7 for an example.

\subsubsection{Shear and Light Attractors}

Now consider the model variants where both a freezing in shear response and a biased swimming toward the preferred light level are included. With the freezing in shear response we get shear attractors as described in section 3.2.8, but now the nature and evolution of these attractors depends on the location of the high shear zone with respect to $z_{L}$. As we saw in section 3.2.7, without a shear response, for slower flows the plankton form a thin layer near $z_{L}$, and for more powerful flows light attractors form, and this is still the case in the low shear portion of the domain. There are two-ways to think about these new structures: either as light attractors whose evolution is interrupted by the freezing in high shear response, or as shear attractors whose evolution depends on their distance from $z_{L}$. For this reason we will call these attractors "shear and light attractors." As was the case for light attractors these can be stable or unstable, depending on whether they contain $z_{L}$ and the center of a gyre.

To better understand the combined effects of the shear response and biased swimming

mechanisms consider the trajectory of a single plankter. As noted in 3.2.8, the swimming decisions of a plankter as they are advected out of a high shear zone are crucial for de- 
termining its trajectory over a long time period afterward, but if the swimming is biased, once they are advected out of a high shear region they immediately start swimming in the direction of $z_{L}$, which essentially gives four possibilities: ${ }^{3}$

1. they are advected out of the top of a high shear region and $z_{L}$ is below them, so they swim back into the high shear region.

2. they are advected out of the top of a high shear region and $z_{L}$ is above them, so they swim farther from the high shear region.

3. they are advected out of the bottom of a high shear region and $z_{L}$ is below them, so they swim farther from the high shear region.

4. they are advected out of the bottom of a high shear region and $z_{L}$ is above them, so they swim back into the high shear region.

\footnotetext{
${ }^{3}$ One might ask about them swimming out "the side" but it is the $z$ axis preference that is important.
} 

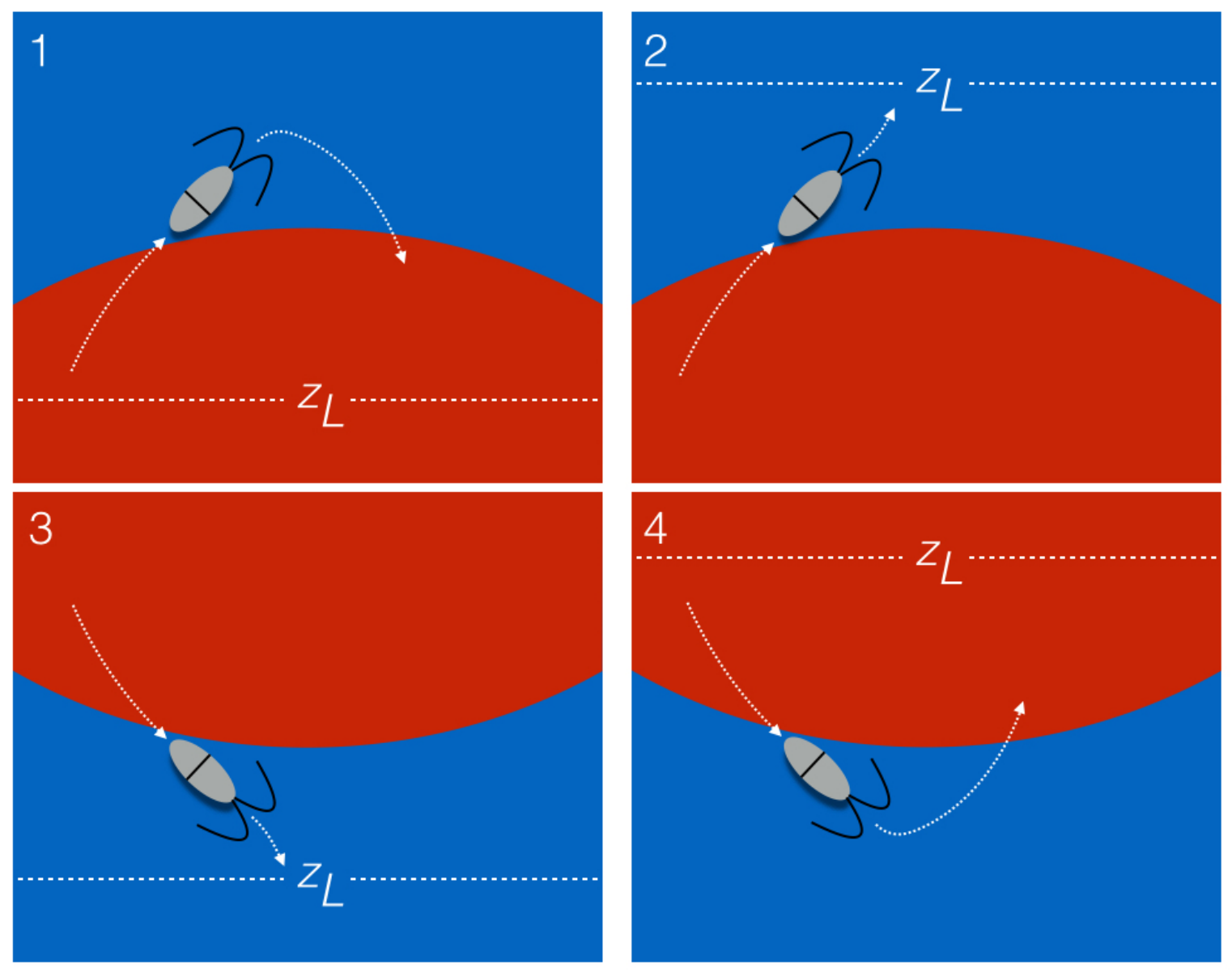

Figure 3.8: The four basic possibilities of a plankter with a preferred light level leaving a high shear region. Red is high shear and blue is low shear.

Figure 3.8 shows these four possibilities. Notice that, in particular, if the high shear region contains $z_{L}$, then rather than swimming in a random direction, they immediately swim back into the high shear region. This is like a shear attractor but even more coherent because the swimming back onto the attractor is guaranteed. If the high shear region does not contain $z_{L}$ then the plankter immediately swims away from it, making it more difficult to create a shear attractor. The similarities to the light attractor cases are clear: if $z_{L}$ is contained in a high shear region we can get a gathering effect analogous to stable light attractors, and if $z_{L}$ is not contained in a high shear region we can get a gathering effect analogous to unstable light attractors. The difference is that the shear response of freezing interrupts the formation of light attractors. An example is given in Figure 3.9. 

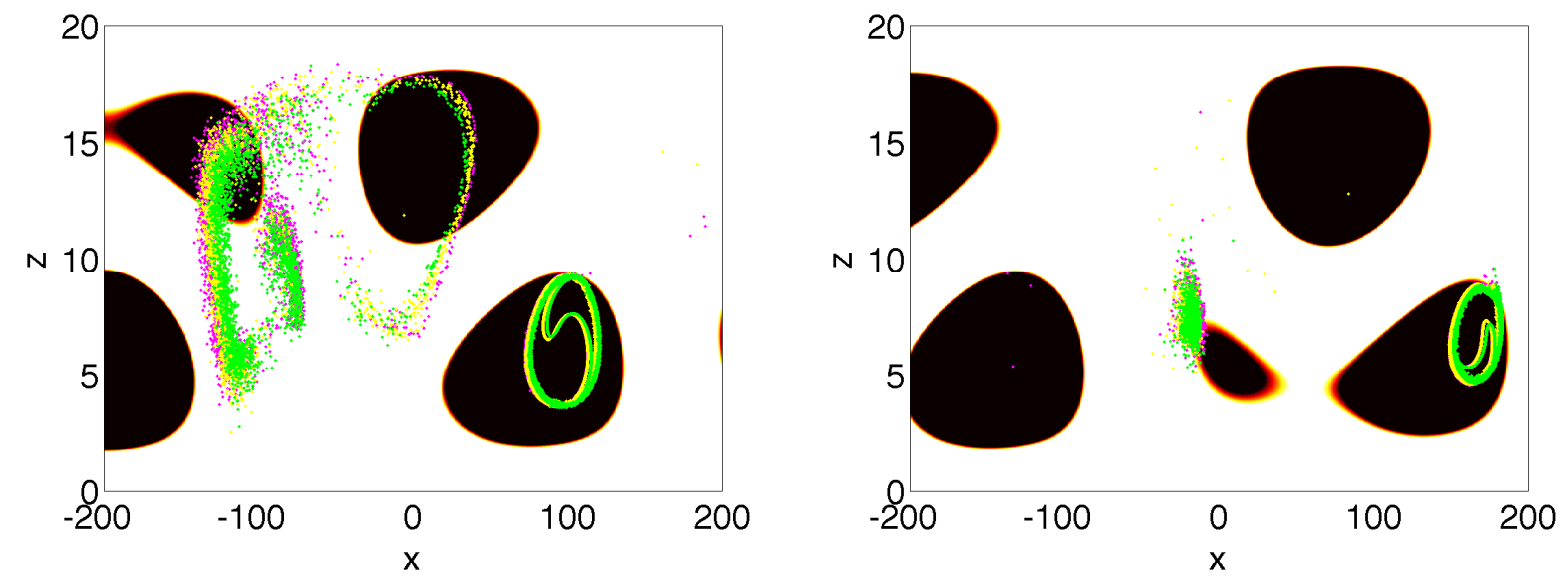

Figure 3.9: The left panel $(t=796 \mathrm{~s})$ shows an unstable and stable attractor pair on the left, and on the right a shear and light attractor containing $z_{L}$. The right panel $(t=1356$ $\mathrm{s}$ ), is after the unstable attractor has been destroyed. The light attractor containing $z_{L}$ has shrunk to match the evolution of the high shear region.

At the level of populations, the interaction of biased swimming and shear response led to a variety of coherent structures. The time evolution of shear and light attractors showed significant variation as well: the shear response can lead to a different number of attractors than without it, it can both increase and decrease the rate of decay of unstable attractors, or it can change the population dynamics to the point where the variants with shear and biased swimming and the variants with biased swimming but without shear bear little resemblence. In simulations it was often the case that a shear attractor would form in a high shear region containing $z_{L}$ and the high shear region would shrink over time, leading to the formation of a stable light attractor. The reverse of this process was also common. For all three models, those flows with less of the domain considered high shear followed the dynamics of the models with biased swimming and no shear response more closely than those flows with more of the domain considered high shear. That is to say, the biased swimming has less of a chance to act when more of the domain is high shear and less swimming actually takes place. As in 3.2.7 we saw these attractors in the fastest four flows considered in all three models with both shear response and biased swimming. 


\subsection{Aggregation and Vertical Patch Formation}

Having outlined the mechanisms present in the various models and how they compare across the range of flows tested, we now consider the major results of this thesis:

- The Stokes' inertia model predicts aggregation of plankton populations along the bottom boundary of high shear regions containing the preferred light level.

- All three models allow formation of plankton patches in the vertical.

\subsubsection{Aggregation Below Regions of High Shear}

The Stokes' inertia model with both biased swimming and a shear response predicts plankton aggregation along the bottom boundary of high shear regions by the following process. As mentioned in section 3.2.3, outside high shear regions in the seven slowest flows, layers of plankton form around $z_{L}$ when the vertical advection is less than $\bar{v}=0.06366 \mathrm{~m} / \mathrm{s}$. This layer formation can be interrupted by high shear regions, and as mentioned in 3.2.6, there is no trapping in gyres in the settling regime, which means these layers of plankton are not advected into ring shaped shear and light attractors, but stay in a more gently deformed layer as they are advected. Since the plankton are initialized at $z_{L}$, partially in a high shear region, at least some of the population immediately begins to settle. This means that for the plankton in high shear, of the four cases depicted in Figure 3.8, the fourth case is the most likely: the particles leave the high shear region at the bottom and $z_{L}$ is above them, so they swim back into the high shear region. However, as soon as they enter the high shear region they stop swimming, leading them to settle once more. As individuals this leads to an oscillation across the bottom boundary of the high shear region, and as a population this leads to an aggregation along the bottom boundary of high shear regions containing $z_{L}$. That is, plankton populations aggregate below high shear regions containing the preferred light level.

Note that since the effect of gravity is the key mechanism for this aggregation to occur and be maintained the fluid particle and neutral buoyancy Stokes' inertia models do not produce these results: these two models may form shear attractors along the bottom boundaries of high shear regions, but without the effect of gravity these shear attractors are advected through the high shear regions with no mechanism to maintain the aggregation 
along the lower boundary. Gravitational effects cannot be ignored, even for these small organisms. In the settling regime the inclusion of gravity introduces a vertical component to the evolution, which in turn yields a significant horizontal separation of the plankton populations of the Stokes' inertia model from the other two models. Figure 3.10 depicts the second slowest flow, where a horizontal separation on the order of 100 meters is created between the model with gravity and the two without.
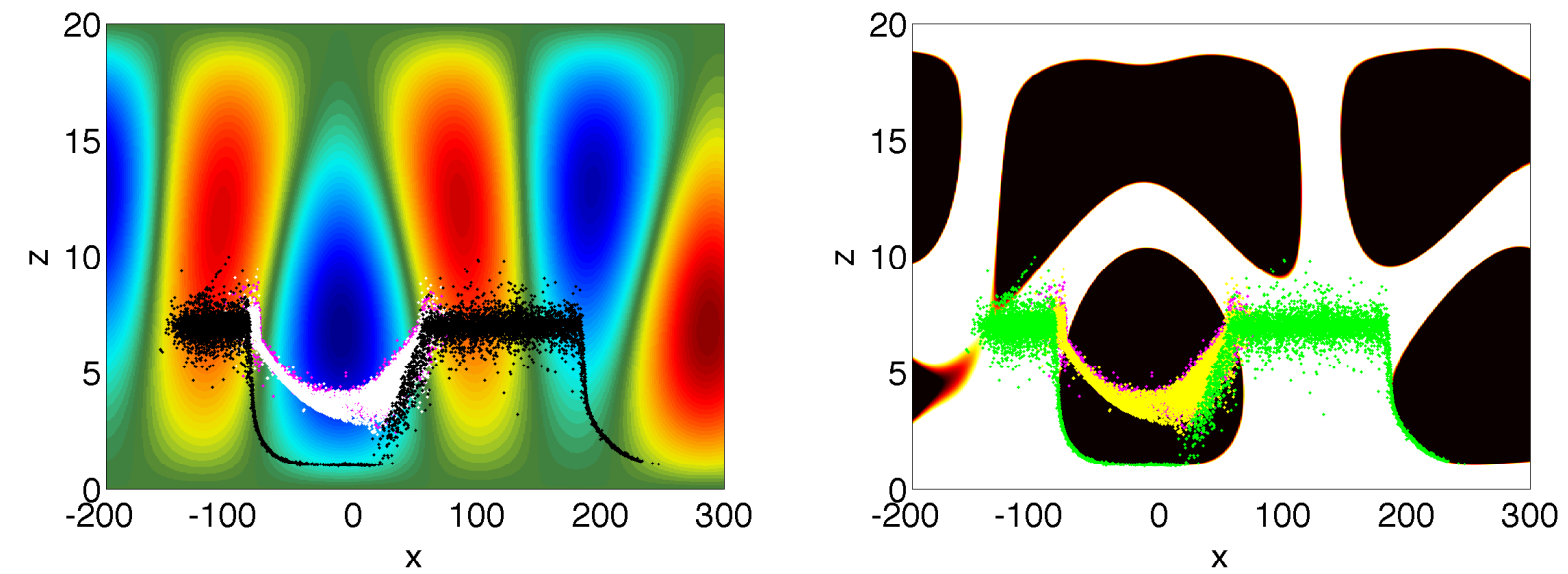

Figure 3.10: Both panels $(t=3920 \mathrm{~s})$ show the aggregation of plankton along the bottom boundary of high shear regions as predicted by the Stokes' inertia model (black in left, green in right). The left panel shows the stream function and the right shows high shear in black. Note the horizontal separation of the fluid particle and neutral buoyancy Stokes' inertia model from the Stokes' inertia model. This substantial separation is a result of gravity acting to separate the populations in the vertical so that the populations are at different locations as the shear regions evolve. In this case the Stokes' inertia model has led to about half the plankton aggregating below high shear, and the other half in two groups at the preferred light level on either side of the other two models.

\subsubsection{Vertical Patch Formation}

Vertical plankton patchiness is mentioned in the literature, but there is not much discussion of its causes. Over large enough scales the shallow water assumption is made, and vertical structure is ignored altogether, so this may at least partially explain this lack of interest. 
The mechanisms of the models considered above are sufficient to generate a variety of vertical structures.
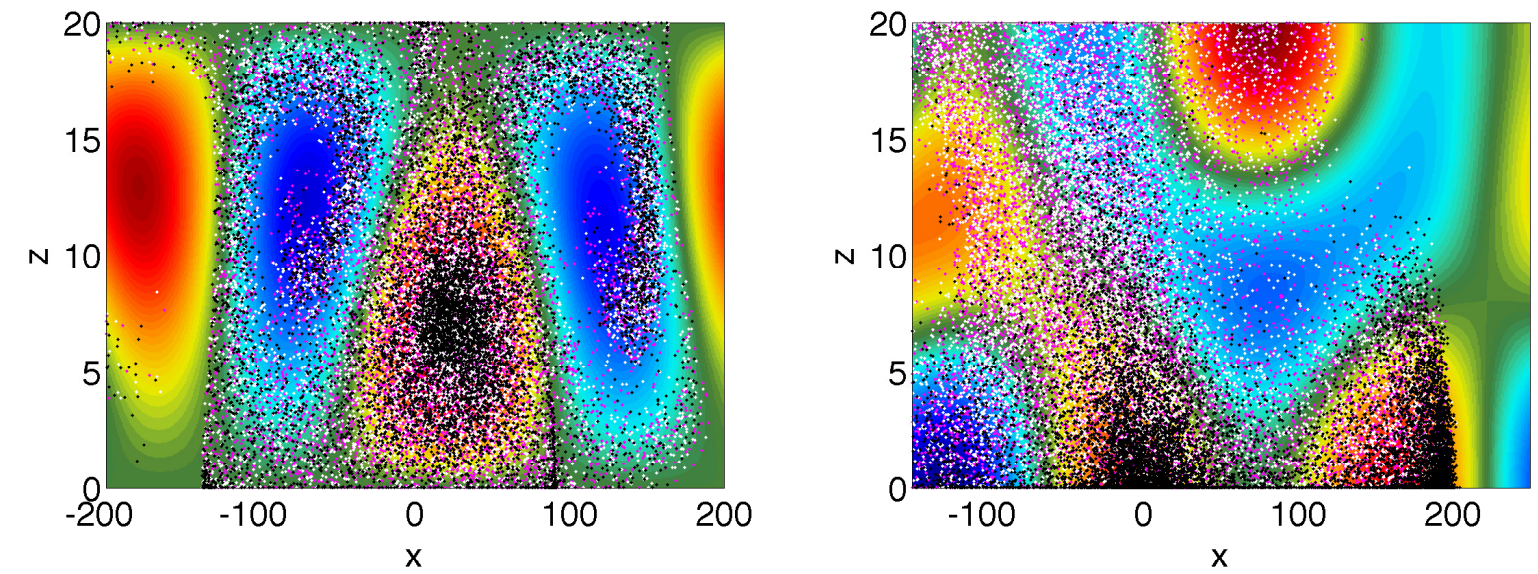

Figure 3.11: Two examples of vertical patch formation in the base models. The left panel $(t=200 \mathrm{~s})$ shows the fastest flow tested. Patches are formed by trapping in gyres, as all populations curl around the blue gyres, and are fairly dense in the central red gyre. Also note the relatively sharp vertical bands at $x=-125,100 \mathrm{~m}$. The right panel $(t=1400$ $\mathrm{s})$ shows the slowest flow where trapping occurred. The background is $u(x, z, t)$, and we clearly see the Stokes' inertia model in black separated to the bottom of the channel and trapped in the horizontal.

For the base models, recall from section 3.2.2 that the eight fastest flows produced trapping in bands, and that some of the fastest flows produce trapped rings of plankton in advected gyres. Both bands and rings are examples of vertical patchiness, as shown in Figure 3.11. For the models with biased swimming only, the primary and secondary light attractors as described in section 3.2.7 are examples of patchiness in the vertical, as depicted in Figure 3.6. For the models with a shear response and no bias in the swimming we have vertical patch formation in great variety. A few examples are given in Figure 3.7. Vertical patch formation is also clear in Figure 3.9 for the models which include both a shear response and biased swimming. 


\section{Chapter 4}

\section{Discussion}

\subsection{Directions for Future Research}

\subsubsection{Light sensing in Zooplankton}

As outlined in [19], the light could act as a cue for plankton in several different ways:

- spectra

- polarization pattern

- absolute intensity

- rate of change of intensity

In our simulations we assumed a constant preferred depth because we were assuming constant light which is reasonable if we assume our simulations do not take place at sunrise or sunset: our simulations were 4000 seconds long, which is about 67 minutes. Care must be taken here as well, because it is tidal forces which cause internal wave trains, and so the relative timing of the lunar and solar cycles is a factor for determining whether the assumption of a constant light level is reasonable: if the internal waves are strongest at sunrise or sunset then assuming constant light levels over an hour which contains the sunrise or sunset is not reasonable. Additionally, a constant light level is a less plausible assumption over longer timescales, for example approaching a day. We also don't know 
which of the four mechanisms listed above is the one which Euphausia pacifica key off of, or if perhaps it is a combination of the mechanisms that matters.

Note that all four of these could be affected by refraction through turbulent waters. It is therefore possible that plankton use light alone to maintain position below high shear regions. Light shining through a high shear region may have a different quality than that shining through a low shear region, and it is possible that the plankton can tell the difference. It is also possible that the difference in light quality confuses the plankton into thinking that they are at their preferred light level, perhaps through a lensing effect which increases the perceived light. This could happen if the plankton are triggered by maximum light intensity rather than average intensity. It seems unlikely that the layers of plankton below high shear regions actually form because they continually swim up, notice it's high shear, and then sink back down.

\subsubsection{Modelling}

First, our model equations for the streamfunction were based on the Euler equations under the Boussinesq approximation linearized about a state of rest. The travelling wave solutions which gave us the streamfunction are therefore a perturbation, and must remain small to be approximately valid. A good measure of "small" is that the fluid speeds remain less than the phase speed, so that there is no wave breaking. However in the parameter space exploration of this paper only the slowest two flows satisfy that criterion. That means that strictly speaking much of our analysis takes place in a non-physical regime. Although the billows of the streamfunction are still qualitatively representative of observed flows, they no longer satisfy the underlying physical requirements of the governing equations. As a result, in the paper we wrote based on this thesis, we did not push the fluid speed much beyond the phase speed. In subsequent simulations we will move to direct numerical simulation so that we may be more confident of the physics.

Second, as we saw in 2.3.2, the Stokes' inertia particle model is based on an equation being applied outside its intended realm of application. However, as described in 2.2, the main effect of using these equations for non-neutrally buoyant cases was that caused by gravity. The more subtle vorticity preference effects were undetectable compared to that

of settling. In the end, then, the Stokes inertia model consists of two terms: one drag, one gravitational. Because gravity is so strong, the main function of the drag term is to have 
settling particles reach the correct terminal settling velocity. By ignoring the imperceptible vorticity preference effects, we can move back to a kinematic model where we simply use the fluid particle model with a settling term in the vertical component:

$$
\begin{aligned}
& \dot{x}=u(x, z, t)+u_{\text {swim }} \\
& \dot{z}=w(x, z, t)+w_{\text {swim }}+w_{\text {settling }}
\end{aligned}
$$

Over the more physical flow speeds just described, the differences in population distribution predicted by this model with biased swimming and freeze in shear and those predicted by the Stokes' inertia model with biased swimming and freeze in shear are imperceptible. This descriptive fluid particle model with settling is much simpler to implement, and gives the same dynamics for the flows we tested. So it seems the effects of inertia on population distribution may be ignored for some sections of parameter space, provided we account for gravity.

\subsection{Conclusions}

The Stokes' inertia model incorporates all the mechanisms considered. In the settling regime this model leads to aggregation of plankton populations below regions of high shear containing the preferred light level. The mechanisms the model is based on are simple enough that we expect this result to be resilient to changes in flow geometry and speeds within the settling regime. Additionally, typical values of density for plankton are greater than that of seawater (see [6], [50], [26]). Therefore any species of plankton with a freeze in shear response and biased swimming towards a preferred light level may be observed in layers below their preferred light level when high shear conditions are met in the settling regime. While the actual critical values for the shear are unknown the critical value choice would change the size of the high shear zones, not their existence. This result should therefore hold over a set of parameters encompassing many geophysical flows and for many species of plankton with a freeze in shear response.

Outside the settling regime, shear and light attractors in the form of rings were observed in the five fastest flows. Since plankton are often observed in nepheloid layers, it is 
reasonable to ask if these simulations are truly representative of the actual flows in which Euphausia pacifica find themselves. The flows relevant to their environment vary with the tide, which means that the slower flows tested may reasonably emulate the flow conditions during periods near slack tide, including the settling regime, where aggregation occurred. The faster flows evolve large shear and light attractors, but it is important to remember that it was assumed the stratification was linear in order to reach these solutions. In reality, a well-defined pycnocline will be present [33]. This means that the restoring force will compress much of the dynamics around this pycnocline, which would likely change the dynamics as well: since the swim speed of the plankton remains unchanged there is no reason to think these structures would scale down without significant structural change. However, in Knight Inlet, our motivating example, the largest change in the pycnocline [33] is far above the preferred light level of Euphausia pacifica [25] and hence the weakly stratified region below the main pycnocline could be approximated by a linear stratification. The case of fully nonlinear waves in a fluid stratified to represent the conditions of Knight Inlet can be simulated numerically in order to address these concerns, and this is something we will return to in future work.

These aggregations only formed for the Stokes' inertia model with biased swimming and a shear response, in the settling regime where the the magnitude of the mean vertical advection was comparable to the settling velocity. As we saw in Figure 3.10, the effect of gravity in this model leads to a separation of the particle cloud, when compared to those produced by the two models without gravitational effects. The mass of an individual plankter is relevant for determining the overall distribution of the population, and as we saw in section 2.1.5, over the range of flows we tested, gravity overshadows the other effects of inertia. Given the similarity of the neutrally buoyant Stokes' inertia and fluid particle models as described in section 3.2.1 it may be possible to adequately describe the motion of plankton in any flow using the fluid particle model with an added gravity term. This would be the cheapest model computationally, but whether it would be the most descriptive depends on whether gravity always overshadows all other effects of inertia for the flows in which Euphausia pacifica find themselves. Thus the consideration of model flows representing more localized features (e.g. localized vortices, shear instability) merits further investigation.

It is not clear that there actually is a freezing in shear response. If the response to shear is to freeze, for plankton to aggregate along the bottom boundary of a high shear region there needs to be mechanisms to push them back onto the boundary from both above and below. Inside the high shear region, provided vertical advection is not too strong, 
gravity pulls the plankton downward to the boundary. Outside the high shear region biased swimming propels the plankton upward to the boundary. If the fluid moves too fast in the vertical it may cancel the downward stabilizing effect of gravity. If it is faster still it may cancel the upward stabilizing effect of biased swimming. However the freeze in shear response was not chosen by Ianson et al. [25] as the most explanatory, but rather they selected a response where the plankton swam down when they encountered the bottom boundary layer, which in their case was synonymous with high shear. If this response was applied to high shear throughout the water column, then swimming would replace gravity as the stabilizing force inside the high shear region. Since swimming $(0.1 \mathrm{~m} / \mathrm{s})$ is much stronger than the settling velocity $(0.005 \mathrm{~m} / \mathrm{s})$, this would lead to aggregation along the bottom boundary of high shear regions for much faster vertical fluid speeds than those in the settling regime. However their model had shear only in the bottom boundary layer, and did not include a shear response elsewhere in the water column. Our models have free-slip boundary conditions, and so no bottom boundary layer, and shear throughout the water column. Because of these differences it may be that a freezing in shear response, rather than a downward swimming response, is adequate to explain the Knight Inlet acoustic data. Again, a test of these models under conditions more closely resembling those of Knight Inlet is required.

If there is a shear response, the critical value of shear at which this response is triggered is unknown. This is a value that could be tuned once we have a simulation of Knight Inlet running, and perhaps reverse engineered through comparison with available acoustic data. While it is conducive to mathematical analysis, our selection of 25,50 , and $75 \%$ quantiles is not realistic in this sense: it is not reasonable to assume that Euphausia pacifica refuse to swim in $1 / 2$ or $3 / 4$ of their environment, although $3 / 4$ could be plausible during energetic transient events such as passing through a KH billow or being struck by an internal wave. Given a realistic set of conditions, one could ask how much of the domain is it reasonable to assume that the plankton consider high shear, or how much of the time is it reasonable to assume that the plankton should be swimming. For now we will be satisfied with our four test values for the critical shear, but it is possible that questions of this type could lead to rough estimates. One could also consider what shear value causes Euphausia pacifica to have difficulty propelling or reorienting themselves, although that problem is currently beyond my abilities to solve.

Throughout the analysis of the numerical experiments we relied heavily on still frames of the animations. It is clear that additional metrics are desirable, but as yet we have found that everything the scalar metrics convey, the animations convey much better. For instance 
it is difficult to express trapping in gyres with a scalar metric, but it is completely clear in animations. We did try several statistical measures, like variance of the particle cloud. We also tried some scalar measures based on the percentage of particles in high shear. Both of these groups of metrics required comparison with the animations to be interpreted correctly, and so were unconvincing by themselves. The greatest intuitive clarity comes from observation of experiment.

When we move to more realistic waves, there is also the question of considering moving to fully three dimensional simulations. As pointed out in [52], two dimensional, steady flows exclude the possibility of chaos, but our flows are time dependent so chaos is possible. Additionally, since plankton are observed in layers, there is good reason to consider two dimensions to be sufficient to represent the plankton component of the model. The preferred light level means that any minor three dimensionality can be overcome by swimming. At the same time, only three-dimensional hydrodynamics can lead to a transition to turbulence. However, given the computational expense and difficulty of rationally analyzing a three dimensional flow, for now a logical next step is to move to more realistic waves and topography, but stay in the two dimensional case. Once such simulations are done, it is entirely possible that we will have a reason to go to three dimensions, for example to consider internal solitary waves with a trapped core (see [32] for numerical simulations and [36] for field observations).

Our work has moved us one rung from the bottom of the hierarchy in 2.1.1. Swimming was also added to the particle model, and we could consider more complicated particle or swimming models in future work. There are many other avenues of possible research we have yet to fully explore. Formal analyses on the interplay of vorticity, relative masses and swim speed in our model could be carried out. A two-dimensional model in the horizontal might be considered important, in which case gravity would be omitted, and vorticity preference effects could become important for some species of plankton. A more formal justification of the inclusion of swimming in our inertial model might yield more nuanced results than those presented here. As a final example, we could include some of the terms we omitted from the Maxey-Riley equations and see how much of a difference they make to the dynamics.

In conclusion the Stokes' inertia model predicts aggregation of plankton populations below high shear regions, as theorized. Our work shows that the mechanisms of advection, gravity, a shear response which can be triggered anywhere in the water column, and biased swimming towards a preferred light level are sufficient to produce population distributions 
similar to those observed. 


\section{APPENDICES}

\subsection{Model Values}

\begin{tabular}{||l||l|l|l||}
\hline \multicolumn{5}{|c|}{ Model Values } \\
\hline Parameter & Symbol & Value & Units \\
\hline horizontal wavenumber 1 & $k_{1}$ & $\frac{2 \pi}{L}=0.0314$ & $\mathrm{~m}^{-1}$ \\
vorizontal wavenumber 2 & $k_{2}$ & $\frac{0.79 \pi}{L}=0.0124$ & $\mathrm{~m}^{-1}$ \\
vertical wavenumber 1 & $m_{1}$ & $\frac{\pi}{H}=0.1571$ & $\mathrm{~m}^{-1}$ \\
buoyancy frequency & $m_{2}$ & $\frac{2 \pi}{H}=0.3142$ & $\mathrm{~m}^{-1}$ \\
frequency (mode 1) & $N_{0}$ & 0.02 & $\mathrm{~s}^{-1}$ \\
frequency (mode 2) & $\sigma_{1}$ & $\frac{N_{0} k_{1} H}{\sqrt{\pi^{2}+k_{1}^{2} H^{2}}}=0.0039$ & $\mathrm{~s}^{-1}$ \\
timestep & $\sigma_{2}$ & $\frac{N_{0} k_{2} H}{\sqrt{4 \pi^{2}+k_{2}^{2} H^{2}}}=7.8938 \times 10^{-4}$ & $\mathrm{~s}^{-1}$ \\
gravitational constant & $d t$ & 0.04 & $\mathrm{~s}$ \\
swim speed & $g$ & 9.81 & $\mathrm{~m} \mathrm{~s}^{-2}$ \\
particle radius & $v_{\text {swim }}$ & 0.1 & $\mathrm{~m} \mathrm{~s}^{-1}$ \\
seawater density & $a$ & 0.004 & $\mathrm{~m}^{-3}$ \\
plankton effective density & $\rho_{f}$ & 1027 & $\mathrm{~kg} \mathrm{~m}^{-3}$ \\
kinematic viscosity & $\rho_{p}$ & 1027.23 & $\mathrm{~m}^{2} \mathrm{~s}^{-1}$ \\
mass of displaced water & $\nu$ & $10^{-6}$ & $\mathrm{~kg}^{-6}$ \\
mass of particle & $m_{f}$ & $\frac{4}{3} \pi a^{3} \rho_{f}=2.7532 \times 10^{-4}$ & $\mathrm{~kg}^{-4}$ \\
mean trajectory duration & $m_{p}$ & $\frac{4}{3} \pi a^{3} \rho_{p}=2.7538 \times 10^{-4}$ & $\mathrm{~s}^{-||}$ \\
diffusion constant & $\zeta$ & $\approx 4$ & $\mathrm{~m}^{2} \mathrm{~s}^{-1}$ \\
Peclet number (base fluid particle) & Pe & $\frac{v_{\text {swim }}^{2}}{2}=0.02$ & 1 \\
\hline
\end{tabular}

Table 4.1: The amplitudes $s$ for the streamfunction vary according to the scheme presented in 3.1 of 3.1.1. The model parameters which are held constant are presented here. The Peclet numbers and diffusion constant are for the base fluid particle model. The inertial model diffusion constant should be similar, 6 with the inertial effects averaging out, but I have not included a proof of this fact. 


\subsection{Classical Analysis: Adding Fluid Drift to Run and Tumble}

In this section we carry out a classical Fokker-Planck analysis of the problem of adding fluid drift to the diffusion of plankton according to the discrete run and tumble model ${ }^{1}$ This type of analysis requires a diffusion constant and Visser [58] appeals to a widely used result that the diffusion constant in $n$ dimensions under all of these assumptions of discrete run and tumble is given by

$$
D=\frac{1}{n} \frac{v_{\mathrm{swim}}^{2} \zeta}{1-\psi}
$$

Where here the reorientation angles are $\theta_{i}$ for the angle chosen after run $i, \psi=\left\langle\cos \left(\theta_{i}\right)\right\rangle$, which measures the correlation between the runs, and $\zeta$ is the mean trajectory duration. Since Visser's model relies completely on the accuracy of this expression in order to make the jump to a Fokker-Planck description, it is worth taking the time to derive it from first principles.

\subsubsection{The Diffusion Constant: One Dimensional Derivation}

For this first derivation we will only consider the case where we're in one dimension and where $\psi=0$. This derivation has the appeal that it does not depend on other results.

For a random (Brownian motion) walk along the $x$ axis with step size $\delta=v_{\text {swim }} t$ where $t$ is the time between events we have

$$
x(N)=x(N-1) \pm \delta
$$

where $N$ is the number of turning events in total time $T$. Squaring this relation, and subsequently averaging, gives us

$$
\begin{aligned}
x^{2}(N) & =x^{2}(N-1) \pm 2 \delta x(N-1)+\delta^{2} \\
\left\langle x^{2}(N)\right\rangle & =\left\langle x^{2}(N-1)\right\rangle+\langle \pm 2 \delta x(N-1)\rangle+\left\langle\delta^{2}\right\rangle \\
\left\langle x^{2}(N)\right\rangle & =\left\langle x^{2}(N-1)\right\rangle+\left\langle\delta^{2}\right\rangle
\end{aligned}
$$

\footnotetext{
${ }^{1}$ This technical discussion is based on a course project for AMATH 777.
} 
where the second term on the right vanishes because we step to the left or right with equal probability. Notice $\left\langle x^{2}(N)\right\rangle=N\left\langle\delta^{2}\right\rangle$ by a simple induction. But now for large total time $T$ we have that $N=\frac{T}{\zeta}$ is a good approximation. Therefore

$$
\left\langle x^{2}(N)\right\rangle=N\left\langle\delta^{2}\right\rangle=\frac{T}{\zeta}\left\langle\delta^{2}\right\rangle=\frac{T}{\zeta} v_{\text {swim }}^{2}\left\langle t^{2}\right\rangle
$$

Since we are assuming that the decision events follow a Poisson process, then the time between events follows an exponential distribution and we have that

$$
\left\langle t^{2}\right\rangle=2\langle t\rangle^{2}=2 \zeta^{2}
$$

so that

$$
\left\langle x^{2}(N)\right\rangle=N\left\langle\delta^{2}\right\rangle=\frac{T}{\zeta} v_{\text {swim }}^{2}\left\langle t^{2}\right\rangle=\frac{T}{\zeta} v_{\text {swim }}^{2} 2 \zeta^{2}=2\left(v_{\text {swim }}^{2} \zeta\right) T
$$

However we also know that $\left\langle x^{2}(N)\right\rangle=2 D T$ for a random walk in one dimension, which means

$$
D=v_{\text {swim }}^{2} \zeta \text {. }
$$

This final expression establishes the one dimensional result.

\subsubsection{The Diffusion Constant: Three Dimensional Derivation}

Now we turn to the source that [5] itself cites as reference, namely [38]. We now follow their derivation for the more general case beginning in section 4 of that paper. Consider a particle diffusing isotropically in $n$ dimensions, then the mean square distance traveled in time $T$ is [8]

$$
\left\langle r^{2}(T)\right\rangle \approx 2 n D T
$$

for large total time $T$ where $D$ is the diffusion constant ${ }^{2}$. We now appeal to [18] a book by Nobel Prize-winning Chemist Paul Flory. In a now standard polymer chemistry argument he develops the formula for the mean end to end distance of a freely rotating polymer chain

\footnotetext{
${ }^{2}$ This is the total variance of displacement, meaning the trace of the covariance matrix. It is a straightforward calculation to show that it takes this form, so we omit it. Mean square distance is a physical interpretation.
} 
with fixed bond lengths and bond angles ${ }^{3}$. Without going into all the details [38] extend this argument to uncorrelated bond angles and lengths. Let $\langle l\rangle$ be the mean bond length and $\left\langle l^{2}\right\rangle$ be the mean square bond length, their arguments lead to

$$
\left\langle r^{2}\right\rangle \approx N\left\langle l^{2}\right\rangle \frac{1+\left(2 \frac{\langle l\rangle^{2}}{\left\langle l^{2}\right\rangle}-1\right) \psi}{1-\psi}
$$

where $N$ is the (large) number of bonds in the chain and as above $\psi$ is the mean cosine between successive bonds. As in the figure, we see that this polymer argument superimposes well onto our image of a run and tumble random walk: bond lengths are run lengths and tumbles are angles between bonds. Therefore we think of $\langle l\rangle$ as the mean run length and $\left\langle l^{2}\right\rangle$ as the mean square run length. Comparing this formula with $\left\langle r^{2}(T)\right\rangle \approx 2 n D T$ we have

$$
D \approx \frac{N\left\langle l^{2}\right\rangle}{2 n T} \frac{1+\left(2 \frac{\langle l\rangle^{2}}{\left\langle l^{2}\right\rangle}-1\right) \psi}{1-\psi}
$$

Again we have the time between decisions as $t$, with $\langle t\rangle=\zeta$, and here again since $N, T$ are large we take the approximation $N=\frac{T}{\zeta}$. If we now make the assumption, as in run and tumble, that $l=v_{\text {swim }} t$ for constant $v_{\text {swim }}$, we have

$$
\begin{aligned}
D & \approx \frac{N v_{\text {swim }}^{2}\left\langle t^{2}\right\rangle}{2 n T} \frac{1+\left(2 \frac{v_{\text {swim }}^{2}\langle t\rangle^{2}}{v_{\text {swim }}^{2}\left\langle l^{2}\right\rangle}-1\right) \psi}{1-\psi} \\
& =\frac{v_{\text {swim }}^{2}\left\langle t^{2}\right\rangle}{2 n \zeta} \frac{1+\left(2 \frac{\zeta^{2}}{\left\langle l^{2}\right\rangle}-1\right) \psi}{1-\psi}
\end{aligned}
$$

but now since $t$ is exponentially distributed we have $\left\langle t^{2}\right\rangle=2 \zeta^{2}$ as above, so that

$$
\begin{aligned}
D & \approx \frac{v_{\mathrm{swim}}^{2} 2 \zeta^{2}}{2 n \zeta} \frac{1+\left(2 \frac{\zeta^{2}}{2 \zeta^{2}}-1\right) \psi}{1-\psi} \\
& =\frac{v_{\mathrm{swim}}^{2} \zeta}{n(1-\psi)}
\end{aligned}
$$

and so Visser uses this $n$ dimensional result

$$
D=\frac{v_{\text {swim }}^{2} \zeta}{n(1-\psi)}
$$

\footnotetext{
${ }^{3}$ Covering all the details of Flory's and Lovely and Dahlquist's arguments is beyond the scope of this outline.
} 
There are far more rigorous derivations available. For instance see [45]. We take the above derivations as sufficient for our purposes. We will also see that we can computationally simulate Visser's run and tumble model, and so avoid the quagmire of references to direct derivations of the diffusion constant.

\subsubsection{Modified Run and Tumble Models: Run and Tumble with Fluid Drift}

We now take Visser's discrete run and tumble model in two dimensions for our swimming, and take the two dimensional drift to be our fluid velocity field $\mathbf{v}=(u, w)$. We can write down the corresponding Langevin equations for the position $(X(t), Z(t))$ of a plankter as

$$
\begin{aligned}
& \frac{d X(t)}{d t}=u(X(t), Z(t), t)+\sqrt{2 D} \Gamma(t) \\
& \frac{d Z(t)}{d t}=w(X(t), Z(t), t)+\sqrt{2 D} \Gamma(t)
\end{aligned}
$$

where $\Gamma(t)$ is Gaussian white noise. This gives us the Fokker-Planck equation

$$
\frac{\partial f(x, z, t)}{\partial t}=-\frac{\partial(f u)}{\partial x}-\frac{\partial(f w)}{\partial z}+D \frac{\partial^{2} f}{\partial x^{2}}+D \frac{\partial^{2} f}{\partial z^{2}}
$$

In our case of two dimensional swimming $(n=2), D$ has the form

$$
D=\frac{1}{2} \frac{v_{\text {swim }}^{2} \zeta}{1-\psi}
$$

but $\psi=\left\langle\cos \left(\theta_{i}\right)\right\rangle$ and we will assume the $\theta_{i}$ are uniformly random, as Visser does for much of his paper. Therefore

$$
\psi=\left\langle\cos \left(\theta_{i}\right)\right\rangle=0
$$

should be a very good approximation for large ensembles. Therefore we can write

$$
D=\frac{1}{2} v_{\text {swim }}^{2} \zeta
$$

and substitute this value into our equations. Note that diffusivity therefore scales as the square of swim speed, and that

$$
\sqrt{2 D}=\sqrt{2 \frac{1}{2} v_{\text {swim }}^{2} \zeta}=v_{\text {swim }} \sqrt{\zeta}
$$


so that our Langevin equation's coefficient on GWN is linearly dependent on the swim speed.

More generally, we have now progressed from a simple geometric model of the swimming pattern of plankton to a correlation time function and diffusion constant $D=$ $D\left(v_{\text {swim }}, \zeta, \psi\right)$. This gives us both the Langevin equations and the Fokker-Planck equation (henceforth FPE) for our swimming model, but requires us to determine $v_{\text {swim }}, \zeta$, and $\psi$.

\subsubsection{FPE for Run and Tumble with Fluid Drift}

Now that we have a FPE, we have obtained a measure of success. However, our FPE has a simple form when written as above, but even if we consider the model where the plankton only swim in the $z$ direction, we get the FPE

$$
\frac{\partial f(x, z, t)}{\partial t}=-\frac{\partial(f u)}{\partial x}-\frac{\partial(f w)}{\partial z}+D \frac{\partial^{2} f}{\partial z^{2}}
$$

which, after taking derivatives of the analytically specified velocity field becomes

$$
\begin{aligned}
\frac{\partial f}{\partial t} & =-\left(-A_{1} \sin \left(k_{1} x-\sigma_{1} t\right) k_{1} \cos \left(m_{1} z\right) m_{1}-A_{2} \sin \left(k_{2} x-\sigma_{2} t+\phi\right) k_{2} \cos \left(m_{2} z\right) m_{2}\right) f \\
& -\left(A_{1} \cos \left(k_{1} x-\sigma_{1} t\right) \cos \left(m_{1} z\right) m_{1}+A_{2} \cos \left(k_{2} x-\sigma_{2} t+\phi\right) \cos \left(m_{2} z\right) m_{2}\right) \frac{\partial f}{\partial x} \\
& -\left(A_{1} \sin \left(k_{1} x-\sigma_{1} t\right) k_{1} \cos \left(m_{1} z\right) m_{1}+A_{2} \sin \left(k_{2} x-\sigma_{2} t+\phi\right) k_{2} \cos \left(m_{2} z\right) m_{2}\right) f \\
& -\left(A_{1} \sin \left(k_{1} x-\sigma_{1} t\right) k_{1} \sin \left(m_{1} z\right)+A_{2} \sin \left(k_{2} x-\sigma_{2} t+\phi\right) k_{2} \sin \left(m_{2} z\right)\right) \frac{\partial f}{\partial z} \\
& +A \frac{\partial^{2} f}{\partial z^{2}}
\end{aligned}
$$

Even this simpler model's FPE appears to be impossible to solve in closed form by hand $^{4}$. Of course the full model's FPE would have even more terms. At this point we have two choices

1. Try a simpler flow which may have a closed form solution.

\footnotetext{
${ }^{4}$ in fact Maple doesn't solve it either
} 


\section{Solve the FPE numerically.}

For point 1, the flow we have selected is already about as simple as it gets if you want the flow to be physical and have a closed form. There is thus no point in passing to an nonphysical flow to do an analysis. We care about plankton swimming in a flow that could actually exist, at least to a good approximation. Therefore we reject option 1.

For point 2, we can absolutely solve the full model's FPE numerically. However this brings up an important point. We did all of this analysis, including assumptions on the swimming of the plankton, appealing to results from polymer chemistry, deriving the diffusion constant, and passing to the FPE and Langevin equations, only to find that we are left with an equation we cannot solve. Is numerically solving this equation really the best step to take? To answer this question, let's consider the model once more.

\subsubsection{Re-evaluating the Model}

Notice that without appealing to the result in [5] about the diffusivity constant that we outlined above there is no obvious direct way to write down a Langevin or Fokker-Planck equation from the discrete run and tumble model because the randomness in the model is based on a Poisson process, not Gaussian white noise.

Lets look at the Langevin equations as a description of the motion with fresh eyes. The equations say that the motion of a plankter is described by the multivariable stochastic process $(X(t), Z(t))$ such that

$$
\begin{aligned}
& \frac{d X(t)}{d t}=u(X(t), Z(t), t)+\alpha \Gamma(t) \\
& \frac{d Z(t)}{d t}=w(X(t), Z(t), t)+\alpha \Gamma(t)
\end{aligned}
$$

for a constant $\alpha$. These equations say that the plankton are being advected by the fluid flow in the $x$ direction just as a deterministic particle would be, but in the $z$ direction they are being advected and are also swimming, where the speed of the swimming depends on $\alpha$.

These are the same equations, but they can be rooted in different assumptions: 
1. Visser assumes the decisions to change direction while swimming follow a Poisson process, so that the tumble times follow an exponential distribution. However we can assume instead that decisions are evenly spaced in time because we've passed to Gaussian white noise.

2. Visser assumes that the speed of the plankton is uniform, but we can assume instead that the plankton swim with speed dictated by $\alpha$. Note that for a particular realization this means that at any given time the speed could be different as we sample increments of the Wiener process and get different values.

That is to say, we could propose an alternate model of plankton swimming based on these equations: that every $\Delta t$ seconds the plankton make a random choice of swim speed and direction according to a Gaussian distribution. So while the run and tumble model

dictates constant speed and varying times between tumbles, this model would make the times between tumbles constant and vary the speed.

This is worth mentioning because in the process of approximating to get these equations we have lost sight of how we have assumed an individual plankter moves. Even though we are, in general, interested in the motion of colonies of plankton, we are also interested in retaining as much information as possible about the swimming of individuals. From a practical standpoint it is important to be able to describe the motion of a single plankton in order to carry out simulations. It also allows the simple simulations to be extend to biologically relevant situations such as predation. Our classical analysis has led us to equations of motion that we cannot solve, and which do not retain enough information for our purposes.

\subsubsection{Run and Tumble with Fluid Drift and Shear Response}

There's one more model we wish to consider before we proceed. It has been observed that some species of plankton will stop swimming if there is sufficient shear at their location in the fluid [25]. This means that as long as the shear remains high enough, say greater than or equal to a constant value $c$, the plankton are passively advected by the flow. The standard way to characterize shear in fluids is by the second invariant of the rate of strain 
tensor [44], which in two dimensions turns out to be

$$
\begin{aligned}
I_{2}(x, z, t) & =\frac{\partial u(x, z, t)}{\partial x} \frac{\partial w(x, z, t)}{\partial z}-\frac{1}{4}\left(\frac{\partial u(x, z, t)}{\partial z}+\frac{\partial w(x, z, t)}{\partial x}\right)^{2} \\
& =\frac{\partial u}{\partial x} \frac{\partial w}{\partial z}-\frac{1}{4}\left(\frac{\partial u}{\partial z}+\frac{\partial w}{\partial x}\right)^{2}
\end{aligned}
$$

We need to cause our plankter to stop swimming if this function exceeds a constant $c$ at its location. It is natural to define the shear response as

$$
R(x, z, t) \equiv 1-H\left(I_{2}(x, z, t)-c\right)
$$

where $H(x)$ is the Heaviside step function. This takes the value 0 whenever the shear is greater than or equal to $c$, and is 1 otherwise. This allows us to define our diffusion constant as

$$
D(x, z, t)=D_{0} R(x, z, t)
$$

where $D_{0}$ is Visser's diffusion constant for 2 dimensions $D_{0}=\frac{v_{\text {swim }}^{2} \zeta}{2}$. Now since the coefficient of white noise in the Langevin equation depends explicitly on position, we get multiplicative noise and spurious drift. We also have a choice to make as to the integration scheme: Ito, Stratonovich, or any of the other continuum of less popular choices available. The real problem though, is that $R$ is not smooth enough for us to have a well-defined FPE no matter what scheme we use. Even if we take the derivative of the Heaviside function to be a delta function, which is itself an approximation, we still need a derivative of the delta function, which leads us into distribution theory and all kinds of considerations beyond the scope of this paper.

We could use some other function instead of $R$. A cutoff function of sufficient smoothness must exist by some standard analysis arguments, such as those used in proving the existence of a partition of unity. Even if we do this we see that we will get a very large and complicated partial differential equation, which of course has no guarantee of having an analytical solution. This equation is even more difficult than the FPE from section 4.4.4 that did not include a shear response, and we could not solve that one either. The hope of making analytic progress on this one seems slim at best.

However, since the point of the Fokker-Planck equation is to get the time dependent probability distribution that describes our process, if we can get that information another way we will be satisfied. We have the following situation: 


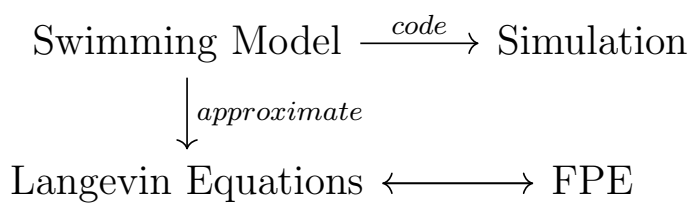

We notice that the classical analysis we carried out above included many assumptions which were made in an attempt to make analytic progress, and yet with all of this approximation and compromise, we were still led to a difficult or perhaps impossible Fokker-Planck equation to solve. Lets reexamine some of the assumptions we've made in an attempt to make analytic progress. Recall from section 2.2.1 that

1. Decisions to change direction while swimming follow a Poisson process, so that the times between tumbles follows an exponential distribution.

- Biologists do not measure the times between tumbles for plankton, this is just an idea. Why should a living thing behave according to the same pattern as radioactive decay?

2. The speed of the plankton while swimming is a constant $v_{\text {swim }}$.

- Plankton are living things, and most living things move at more than one speed. Even if we picked two discrete times at which our plankton moved, it could vastly complicate our model.

3. Each plankter moves independently of all others. The plankton do not interact.

- This may be true if they are sufficiently spread out, but there is no particular reason to think this is true. As a limiting case, a high enough concentration of them would mean they were physically in each other's way.

We can see from this list that the real problem with this model is that the plankton, though simple creatures, have agency. They get tired, so in some sense their position process is not really Markovian, and this entire analysis is questionable. They search for food, which may not be evenly distributed in their environment. They do not turn instantaneously or travel in perfectly straight lines. Their agency poses a challenge to modelling their group behaviour.

No model is perfect, so one may object to our picking it apart. After all, a model that is a perfect representation of nature loses its usefulness, because it is just as complicated. 
A model must simplify what it represents in such a way that it retains the information that we deem to be most important. The run and tumble model seems more like a model of opportunity in the sense that the polymer chain argument could be adapted by making the observation that if constant speed was assumed then the length of the bonds could be replaced by swim trajectories. This was done before the advent of cheap computing power, and is understandable as it gave those involved a way to make analytic progress.

The point here is that we made many assumptions in order to get to our diffusion constant, which puts the value of that constant in serious doubt. We were unable to make analytic progress on the standard model, and as soon as we add another factor, in this case the shear response, we get a Fokker-Planck equation that is even more intractable. So as valuable as all of this classical analysis can be, we see that the process of making assumptions in order to make analytic progress has not been a worthwhile compromise in this case because in the end we were not able to make enough analytic progress to actually get to our answer.

We can see that there is room for a better model of plankton motion that makes different assumptions and in fact there are more models, but examining and comparing all of them or developing a new model is beyond the scope of this paper. Instead we focus on discrete run and tumble with all of its imperfections, and set ourselves the task of finding the information that the probability density function from the Fokker-Planck equation would provide.

We will proceed using simulation. It is true that we could simulate the associated Langevin equations to the Fokker-Planck equation we cannot solve, but we can simulate their motion according to the run and tumble assumptions directly, so there is no reason to. We could also numerically attempt to solve the Fokker-Planck equation, but again, after viewing the assumptions made and the resulting compromises, it is much more direct to simply simulate the swimming model. For this reason we reject option 2: numerical integration of the FPE mentioned in section 4.4.4.

\subsubsection{Conclusion}

The limitations of the classical approach are now clear. Even the simple case of adding a shear response halted our progress. For this reason we abandoned this approach early on. 
Additionally, we wish to consider arbitrary starting geometries and the effects of inertia, neither of which we attempted in the classical framework, but which would also complicate the analysis. It is for all these reasons that this analysis features in the appendix rather than in the main body of the work.

\subsection{On the Inadequacy of Variance for our Problem}

One classical metric when dealing with a randomly walking population is to consider the (total) variance ${ }^{5}$ as a function of time. In the classical analysis if the initial conditions of the Fokker-Planck equation are taken to be a delta function of position, so that it is assumed the initial position of the plankton is a single point, without drift the motion of the population is like a diffusive process, featuring a linear increase of variance over time. It is unsurprising that one parameter should capture the growth of a diffusion process because of the isotropy of the diffusion. The introduction of a background drift can have profound consequences on the geometry of a diffusion population, yet it is sometimes claimed in the plankton literature, for example see [59], that on a large scale, this linear increase idea still applies to the motion of plankton. This view leads to some obvious questions:

1. What about drift? In the case of plankton motion, the drift is given primarily by the motion of the seawater in which they live. There are an infinite variety of possible drift configurations. Why would we think that a one-parameter idea like variance as a function of time could capture anything meaningful about all of them?

2. Does variance increase linearly with time? Over the points in parameter space we tested, a linear increase of variance over time was not the norm. Those plots that looked most linear increased at a different rate than predicted by the theory. We found that the linear increase over time idea was not even helpful for the sake of intuition.

3. What do we mean by "large scale"? If we consider large enough scales we can consider the location of a group of plankton as a point, and perhaps average out all the flow detail to get the perspective that plankton spread in the ocean as dye diffuses in a still glass of water. However at these scales the relatively slow swimming of plankton means they can be approximated as passive tracers.

\footnotetext{
${ }^{5}$ That is, the trace of the covariance matrix
} 
We found variance to be essentially useless as a metric for comparing or analyzing runs. The one thing we could say is that at our scales the idea of treating the plankton motion as a diffusion process was woefully inadequate to describe the results of our simulations. There are papers which study the variance as a function of wavenumber in Fourier space, see [47] for an overview. We may do the same in the future. What we did find, was that the linear increase of variance over time was not helpful in our analyses, and that any anomaly in the variance plots required us to return to the animations for an explanation anyway. In this situation variance plots were an analytic tool with no predictive or descriptive power. 


\section{References}

[1] E. Achenbach. Experiments on the flow past spheres at very high Reynolds numbers. Journal of Fluid Mechanics, 54(3):565, 1972.

[2] Y. D. Afanasyev and W. R. Peltier. On breaking internal waves over the sill in knight inlet. Proceedings of the Royal Society of London A: Mathematical, Physical and Engineering Sciences, 457(2016):2799-2825, 2001.

[3] A. Babiano, J. H. E. Cartwright, O. Piro, and A. Provenzale. Dynamics of a small neutrally buoyant sphere in a fluid and targeting in hamiltonian systems. Phys. Rev. Lett., 84:5764-5767, Jun 2000.

[4] K. Banse. On the vertical distribution of zooplankton in the sea. Progress in Oceanography, 2:53 - 125, 1964.

[5] H. C. Berg. Random Walks in Biology. Princeton University Press, Princeton, NJ, 1992.

[6] R. W. Campbell and J. F. Dower. Role of lipids in the maintenance of neutral buoyancy by zooplankton. Marine Ecology Progress Series, 263:93-99, 2003.

[7] J. H. E. Cartwright, U. Feudal, G. Károlyi, A. de Moura, O. Piro, and T. Tél. Dynamics of finite-size particles in chaotic fluid flows. Understanding Complex Systems, page $51,2010$.

[8] S. Chandrasekhar. Stochastic problems in physics and astronomy. Reviews of Modern Physics, 15(1), 1943.

[9] C. T. Crowe, T. R. Troutt, and J. N. Chung. Numerical models for two-phase turbulent flows. Annual Review of Fluid Mechanics, 28(1):11-43, 1996. 
[10] P. F. Cummins. Stratified flow over topography: time-dependent comparisons between model solutions and observations. Dynamics of Atmospheres and Oceans, 33(1):43 72,2000 .

[11] P. F. Cummins, S. Vagle, L. Armi, and D. M. Farmer. Stratified flow over topography: upstream influence and generation of nonlinear internal waves. Proceedings of the Royal Society of London A: Mathematical, Physical and Engineering Sciences, 459(2034):1467-1487, 2003.

[12] A. De Robertis, C. Schell, and J. S. Jaffe. Acoustic observations of the swimming behavior of the euphausiid euphausia pacifica hansen. ICES Journal of Marine Science: Journal du Conseil, 60(4):885-898, 2003.

[13] W. M. Durham, E. Climent, M. Barry, F. De Lillo, G. Boffetta, M. Cencini, and R. Stocker. Turbulence drives microscale patches of motile phytoplankton. Nature Communications, 4, July 2013.

[14] W. M. Durham, E. Climent, and R. Stocker. Gyrotaxis in a steady vortical flow. Phys. Rev. Lett., 106:238102, Jun 2011.

[15] W. M. Durham, J. O. Kessler, and R. Stocker. Disruption of vertical motility by shear triggers formation of thin phytoplankton layers. Science, 323(5917):1067-1070, 2009.

[16] D. Farmer and L. Armi. The generation and trapping of solitary waves over topography. Science, 283(5339):188, 1999.

[17] D. M. Farmer and L. Armi. Stratified flow over topography: models versus observations. Proceedings of the Royal Society of London A: Mathematical, Physical and Engineering Sciences, 457(2016):2827-2830, 2001.

[18] P. J. Flory. Statistical Mechanics of Chain Molecules. Wiley, New York, NY, 1969.

[19] T. M. Frank and E. A. Widder. The correlation of downwelling irradiance and staggered vertical migration patterns of zooplankton in wilkinson basin, gulf of maine. Journal of Plankton Research, 19(12):1975, 1997.

[20] Y.-C. Fung. A First Course in Continuum Mechanics: Second Edition. Prentice-Hall, New Jersey, 1977.

[21] A. E. Gill. Atmosphere-ocean dynamics, volume 30. Academic press, 1982. 
[22] C. F. Greenlaw. Backscattering spectra of preserved zooplankton. The Journal of the Acoustical Society of America, 62(1):44-52, 1977.

[23] G. Haller and T. Sapsis. Where do inertial particles go in fluid flows? Physica D: Nonlinear Phenomena, 237(5):573 - 583, 2008.

[24] M. S. Hoecker-Martínez and W. D. Smyth. Trapping of gyrotactic organisms in an unstable shear layer. Continental Shelf Research, 36:8, 2012.

[25] D. Ianson, S. E. Allen, D. L. Mackas, M. V. Trevorrow, and M. C. Benfield. Response of Euphausia pacifica to small-scale shear in turbulent flow over a sill in a fjord. Journal of Plankton Research, 33(11):1679, 2011.

[26] W. Jacobs. Das Schweben der Wasserorganismen, pages 131-218. Springer Berlin Heidelberg, Berlin, Heidelberg, 1935.

[27] J. M. Klymak and M. C. Gregg. Three-dimensional nature of flow near a sill. Journal of Geophysical Research: Oceans, 106(C10):22295-22311, 2001.

[28] J. M. Klymak and M. C. Gregg. The role of upstream waves and a downstream density pool in the growth of lee waves: Stratified flow over the knight inlet sill. Journal of Physical Oceanography, 33(7):1446-1461, 2003.

[29] J. M. Klymak and M. C. Gregg. Tidally generated turbulence over the knight inlet sill. Journal of Physical Oceanography, 34(5):1135-1151, 2004.

[30] T. Knutsen, W. Melle, and L. Calise. Determining the mass density of marine copepods and their eggs with a critical focus on some of the previously used methods. Journal of Plankton Research, 23(8):859-873, 2001.

[31] P. K. Kundu, I. M. Cohen, and D. R. Dowling. Fluid Mechanics: Fifth Edition. Academic Press, Oxford, 2012.

[32] K. G. Lamb. Shoaling solitary internal waves: on a criterion for the formation of waves with trapped cores. Journal of Fluid Mechanics, 478:81-100, 32003.

[33] K. G. Lamb. On boundary-layer separation and internal wave generation at the Knight Inlet sill. Proceedings: Mathematical, Physical and Engineering Sciences, 460(2048):2035, 2004.

[34] W. Lampert. The adaptive significance of diel vertical migration of zooplankton. Functional Ecology, 3(1):21-27, 1989. 
[35] C. E. Lennert-Cody and P. J. S. Franks. Plankton patchiness in high-frequency internal waves. Marine Ecology-Progress Series, 186:59-66, 1999. n/a.

[36] R.-C. Lien, E. A. D'Asaro, F. Henyey, M.-H. Chang, T.-Y. Tang, and Y.-J. Yang. Trapped core formation within a shoaling nonlinear internal wave. Journal of Physical Oceanography, 42(4):511-525, 2015/10/27 2011.

[37] E. Loth. Numerical approaches for motion of dispersed particles, droplets and bubbles. Progress in Energy and Combustion Science, 26(3):161 - 223, 2000.

[38] P. Lovely and F. Dahlquist. Statistcal measures of bacterial motility and chemotaxis. Journal of Theoretical Biology, 50, 1975.

[39] D. Mackas, H. Sefton, and C. Miller. Vertical habitat partitioning by large calanoid copepods in the oceanic subarctic pacific during spring. Progress in Oceanography, 32:259, 1993.

[40] M. R. Maxey. The gravitational settling of aerosol-particles in homogeneous turbulence and random flow-fields. Journal of Fluid Mechanics, 174:441, 1987.

[41] E. E. McPhee-Shaw and E. Kunze. Boundary layer intrusions from a sloping bottom: A mechanism for generating intermediate nepheloid layers. Journal of Geophysical Research: Oceans, 107(C6):3-1-3-16, 2002.

[42] M. J. Morris, G. Gust, and J. J. Torres. Propulsion efficiency and cost of transport for copepods: a hydromechanical model of crustacean swimming. Marine Biology, 86(3):283-295, 1985.

[43] Y. Nakagawa, Y. Endo, and H. Sugisaki. Feeding rhythm and vertical migration of the euphausiid euphausia pacifica in coastal waters of north-eastern japan during fall. Journal of Plankton Research, 25(6):633-644, 2003.

[44] J. Olsthoorn, M. Stastna, and D. Steinmoeller. On the dynamics of vortex-wall interaction in low viscosity shear thinning fluids. Physics of Fluids, 26, 2014.

[45] C. S. Patlak. Random walk with persistence and external bias. Bulletin of Mathematical Biophysics, 15, 1953.

[46] T. J. Pedley and J. O. Kessler. Hydrodynamic phenomena in suspensions of swimming microorganisms. Annual Review of Fluid Mechanics, 24(1):313-358, 1992. 
[47] T. M. Powell and A. Okubo. Turbulence, diffusion, and patchiness in the sea. Philosophical Transactions: Biological Sciences (Generalizing across Marine and terrestrial Ecology), 343(1303), 1994.

[48] L. B. Quetin. Depth distribution of developing euphausia superba embryos, predicted from sinking rates. Marine biology. 79(1). Germany, Vol. 79(no. 1):P.47-53, 1984.

[49] B. Rothschild and T. Osborn. Small-scale turbulence and plankton contact rates. Journal of Plankton Research, 10:465, 1988.

[50] J. A. Rudjakov. The possible causes of diel vertical migrations of planktonic animals. Marine Biology, 6(2):98-105, 1970.

[51] T. P. Sapsis, N. T. Ouellette, J. P. Gollub, and G. Haller. Neutrally buoyant particle dynamics in fluid flows: Comparison of experiments with lagrangian stochastic models. Physics of Fluids, 23, 2011.

[52] A. Scotti and J. Pineda. Plankton accumulation and transport in propagating nonlinear internal fronts. Journal of Marine Research, 65:117, 2007.

[53] J. H. Simpson and J. Sharples. Introduction to the physical and biological oceanography of shelf seas. Cambridge University Press, 2012.

[54] K. Squires and H. Yamazaki. Preferential concentration of marine particles in isotropic turbulence. Deep-Sea Research Part I: Oceanographic Research Papers, 42(11-12):1989-2004, 1995.

[55] M. Stastna and W. R. Peltier. Upstream-propagating solitary waves and forced internal-wave breaking in stratified flow over a sill. Proceedings of the Royal Society of London A: Mathematical, Physical and Engineering Sciences, 460(2051):3159-3190, 2004.

[56] M. Stastna, F. J. Poulin, C. Subich, and J. V. Mecking. The effect of stochastic perturbations on plankton transport by internal solitary waves. Nonlinear Processes in Geophysics, 18, 2011.

[57] R. D. Vilela, A. P. S. de Moura, and C. Grebogi. Finite-size effects on open chaotic advection. Phys. Rev. E, 73:026302, Feb 2006.

[58] A. W. Visser. Lagrangian modeling of plankton motion: From deceptively simple random walks to Fokker-Planck and back again. Journal of Marine Systems, 70:287, 2008 . 
[59] A. W. Visser and U. H. Thygesen. Random motility of plankton: diffusive and aggregative contributions. Journal of Plankton Reserach, 25(9):1157, 2003.

[60] E. Wallner and E. Meiburg. Vortex pairing in two-way coupled, particle laden mixing layers. International Journal of Multiphase Flow, 28:325, 2002.

[61] J. Yen, K. Rasberry, and D. Webster. Quantifying copepod kinematics in a laboratory turbulence apparatus. Journal of Marine Systems, 69:283, 2008. 